\title{
MOLD MATERIALS FOR PERMANENT MOLD CASTING OF ALUMINUM ALLOYS
}

\section{Final Report}

John F. Wallace

David Schwam

Wen Hong

Case Western Reserve University

September 2001

Work Performed Under Contract DE-FC07-99ID13718

For

U.S. Department of Energy

Assistant Secretary for

Energy Efficiency and Renewable Energy

Washington D.C. 


\section{TABLE OF CONTENTS}

I. ABSTRACT

II. BACKGROUND

PART 1 -MOLD MATERIALS

1.1 Introduction 6

1.2 The Thermal Fatigue Test 8

1.3 Evaluated Mold Materials 11

1.4 Results and Discussion $\quad 12$

1.5 Conclusions 15

$\begin{array}{ll}1.6 \text { References } & 17\end{array}$

PART 2 - EVALUATION OF COATINGS FOR PERMANENT MOLDS

2.1 Introduction 18

2.2 Experimental Equipment and Procedures 31

2.3 Results of the Evaluation and Discussion 39

2.4 Conclusions $\quad 52$

2.5 References 54

FIGURES AND TABLES FOR PART 1

FIGURES AND TABLES FOR PART 2

APPENDICES 


\section{ACKNOWLEDGEMENT}

The support of the Department of Energy, Office of Industrial Technology and the Cast Metals Consortium Program for this work is gratefully acknowledged. The work was conducted through the Permanent Mold Committee of the American Foundry Society (AFS Committee 2-E), Chairman Jay Laughlin.

Thanks are due to Mr. Roy Evers of Saint Louis Precision Casting Company for providing cast iron and steel specimens. Thanks are also due to Mr. Philip Sanford and Mr. Robert Pischel of Foseco for providing permanent mold refractory coatings and valuable inputs in the interpretation of the experimental data.

This publication was prepared with the support of the U.S. Department of Energy (DOE) Award No. DE-FC07-99ID13718 . However, any opinions, findings, conclusions, or recommendations expressed herein are those of the author(s) and do not necessarily reflect the views of DOE. 


\section{ABSTRACT}

A test that involves immersion of the potential mold materials for permanent molds has been developed that provides a thermal cycle that is similar to that experienced during casting of aluminum in permanent molds. This test has been employed to determine the relative thermal fatigue resistance of several different types of mold materials. Four commercial mold coatings have been evaluated for their insulating ability, wear resistance and roughness.

The results indicate that composition and structure of the mold materials have a considerable effect on their thermal fatigue cracking behavior. Irons with a gray iron structure are the most prone to thermal fatigue cracking followed by compacted graphite irons with the least thermal fatigue cracking of the cast irons experienced by ductile iron. The composition of these various irons affects their behavior. Cast steels with an H13 steel composition provide the least cracking of the cast materials. Cast 4140 steel shows a tendency to produce a considerable amount of cracking compared to cast H13. The best resistance to thermal fatigue cracking is obtained with wrought $\mathrm{H} 13$ steels produced to a premium grade and heat treated to 47 Rockwell C. The evaluation of the coatings shows that they can be used for different purposes to provide good, intermediate or low insulation with improved casting release properties. 


\section{BACKGROUND}

Experience with metal molds for aluminum permanent mold casting has established that the primary cause for failure is thermal fatigue cracking[1]. These cracks occur because of the repetitive heating and cooling of the mold surface. The cyclic heating and cooling creates conditions that promote thermal fatigue cracking of the die surface. These cracks can become deep and numerous and result in the need to replace the die or mold after a period of time.[2,3,4]

The normal temperature cycle for permanent molds differs significantly from die casting dies. Die casting dies are water cooled with water lines that generally approach one inch below the die surface. These cooling lines plus the water spray that carries the lubricant cool the surface to a relatively low temperature before the next cycle. When thermocouples are placed at the surface in die casting, the temperatures reach $1100^{\circ} \mathrm{F}$ to $1200^{\circ} \mathrm{F}\left(593-649^{\circ} \mathrm{C}\right)$. The surface of the die is coated with a lubricant that is normally a dilute solution in a water carrier. This lubricant degenerates and over a period of time builds up a degenerate lubricant-oxide layer on the die surface. The water carrier spray effectively quenches the die surface.

The permanent mold surface is coated with a ceramic wash that is semipermanent. The properties of this wash vary from insulating to more conductive 
depending on the feeding paths for the casting but the wash is generally more insulating than the die lubricant layer on the die casting die[4,5]. Four of these washes were studied in this paper.

The body of the permanent mold is usually higher in temperature than the die casting die. This mold can be either water or air cooled but this cooling is less effective, often reducing the temperature of the mold less than the water cooling of the die casting die, so the body of the mold is somewhat hotter than in a die casting die. However, the mold wash provides a more effective barrier to heat transfer than lubricant-oxide layer on the die casting die.

The net result is a somewhat reduced temperature range or $\Delta \mathrm{T}$ for the permanent mold than for the die, particularly at the surface. The reduced $\Delta \mathrm{T}$ and the lower surface temperature attained in the permanent mold provide less severe thermal fatigue conditions than for die casting. Permanent molds are frequently made of gray iron; die casting dies of cast irons are not employed because their life would be short. 


\section{PART 1}

\section{MOLD MATERIALS FOR PERMANENT MOLDS}

\section{INTRODUCTION}

This final report is for a CMC-DOE-AFS-sponsored research project (AFS Committee 2-E), undertaken to provide an evaluation of the relative mold life of metallic permanent molds used for casting aluminum. The objective was to permit an optimized selection of molds to be used for a given casting depending on size, shape and required quantity of those castings. In this way, the cost of the molds could be reduced without sacrificing the surface quality of the castings and still maintain a low cost for the molds. It is pointed out that the ultimate cost of the molds is affected by a number of factors. These include the life of the mold, finishing cost of the castings and the machining cost of the mold, as well as the cost of mold materials.

At the present, the metal molds used in permanent molding of aluminum alloys are selected from a fairly large field of materials, ranging from gray iron to high-strength tool steels. The selection of the mold material is based primarily on the prior history of the foundry, experience of the operators and number and type of castings to be produced. Systematic information on the relative behavior of potential mold materials under thermal fatigue conditions is lacking, so, at times, it may be difficult to select the optimum mold 
material for a given type and number of castings. As a result, mold life varies significantly, affecting the cost of the castings.

The usual mode of failure of permanent molds for aluminum casting is thermal fatigue cracking produced by the cyclic thermal stress on the mold face [1]. Resistance to thermal fatigue can be increased by using mold materials that have a combination of high thermal conductivity, high strength at elevated temperature, low coefficient of thermal expansion and a low modulus of elasticity.

The relative mold life under the thermal conditions that prevail in permanent molds that experience exposure to molten aluminum was investigated for a range of materials such as gray iron (Meehanite), ductile iron and compacted graphite iron. These materials may be used plain or alloyed to provide microstructures with different life and stabilities at elevated temperature. Other candidate materials to be tested are cast $4140-$ type steels and cast and wrought H13 steels. Some recent studies conducted by the authors on die materials for aluminum die casting have shown the advantages of premium grade $\mathrm{H} 13$ wrought steel that is rapidly cooled from the austenitizing temperature and double tempered to 46-48 Rockwell C hardness. The wrought $\mathrm{H} 13$ steel evaluated in this investigation was the P.G. H13, oil quenched and tempered to 47 Rockwell C for long mold life. 


\subsection{THE THERMAL FATIGUE TEST}

A test for permanent mold materials that is similar to the die casting die test with a less severe testing cycle, has been developed for the evaluation of the thermal fatigue resistance of permanent mold materials. The thermal fatigue specimens are processed to the dimensions shown in Fig. 1A. It is a $2 \times 2 \times 7-$ in. $(5.1 \times 5.1 \times 18-\mathrm{cm})$ rectangular parallelpiped specimen with a 1.25 -in. $(3.2-\mathrm{cm})$ dia, 6.5 -in. $(16.5-\mathrm{cm})$ deep hole in the center for internal water cooling. The test produces considerable constraint and considerable thermal fluctuations during immersion and removal from the 356 aluminum alloy bath held at $1350^{\circ} \mathrm{F}\left(732^{\circ} \mathrm{C}\right)$, used for permanent mold materials. The test equipment is also illustrated in Fig. 1B.

The four corners of the thermal fatigue specimen have a 0.010 -in. $(0.025-\mathrm{cm})$ radius that intensifies the predominately uniaxial stress at this location. Although the outer surface of the diecasting test specimen is sprayed with a commercial water-base lubricant just before it enters the molten $\mathrm{Al}$ bath, it was decided not to use this spray or any coating for permanent mold materials. The effect of coatings on the heat transferred from the molten aluminum to the specimen was evaluated for the coatings in a separate part of this project. 
For the testing of potential materials for both aluminum die casting and permanent molds, water flows through the specimen at a rate of 1.5 gallons per minute. The test cycle is shown in Fig. 2. The specimen is immersed for eight seconds for permanent mold materials, compared to 12 seconds for diecasting die materials. Then the permanent mold material test specimen is removed from the bath and allowed to cool in air for 42 seconds for permanent mold materials, compared to only 24 seconds including spray time for diecasting dies. The severe conditions of testing that apply to the aluminum die casting process were altered to less severe conditions that more closely fit the permanent mold.

The result of these changes was to decrease the maximum temperature obtained at the corners of the specimen and to lengthen the cycle. The difference in the thermal cycle at the corners is illustrated in Fig. 3 for thermocouples that are located at $0.10 \mathrm{in}$. $(0.25$ $\mathrm{cm}$ ) from the surface. The maximum temperature reached at this location was nearly $1000^{\circ} \mathrm{F}\left(538^{\circ} \mathrm{C}\right)$ for the die cast specimens and nearly $800^{\circ} \mathrm{F}\left(427^{\circ} \mathrm{C}\right)$ for the permanent mold test. These readings were taken on a thermocouple located inside a 0.08 -in. $(0.20$ $\mathrm{cm}$ ) diameter hole at the specimen corner. The centerline of the hole was located at 0.10 in. $(0.25 \mathrm{~cm})$ from the outer surface of the specimen, and the thermocouple was located at 4 inches $(10 \mathrm{~cm})$ from the top of the 7 -in. $(18-\mathrm{cm})$ long specimen.

The relative thermal fatigue resistance of potential mold materials for aluminum permanent mold casting has been determined by measuring the cracks that occur on the 
middle three-inch length of the test piece after up to 5,000 cycles. The cracks have been evaluated both by:

1. Average Maximum Crack Length - average of the maximum crack length on each of the four corners

2. Total Crack Area - obtained by multiplying the number of cracks by the square of their length, and by adding the sum of the products. This calculation is assuming that all cracks continued across the corner to the other side.

This procedure was followed for the permanent mold tests, the measurements made after cycles of $1,000,2,000,3000,4,000$ and 5,000 using the immersion cycle shown for permanent molds in Figure 2.

The thermal fatigue testing cycle for permanent mold material was developed by utilizing a series of different times of immersion into the 356 alloy at $1350^{\circ} \mathrm{F}\left(732^{\circ} \mathrm{C}\right)$ and different holding periods in forced air with water cooling on the specimen. The purpose of the tests was to establish a testing cycle that would be closer to the thermal cycle of a permanent mold used for aluminum castings, and still provide a measurement of the relative thermal fatigue resistance in a reasonably short testing cycle. This cycle had to allow for the insulating effect of the semi-permanent mold wash or coating and the absence of the die lubrication in the water carrier. 
In general, the cycle time for each casting is usually longer for each permanent mold than for the die casting of aluminum. It is also necessary that the testing cycle be relatively severe within the aforementioned limits to accentuate the thermal fatigue cracking and produce meaningful test results in a reasonable length of time. Figure 3 illustrates the thermal fluctuations that were obtained as measured at 0.10 in. $(0.25 \mathrm{~cm})$ from the specimen surface. The time for each cycle is 50 seconds, compared to a shorter period of 36 seconds for the die casting test. The maximum temperature is also lower.

\subsection{EVALUATED MOLD MATERIALS}

A total of ten specimens have been tested. All were of the same dimensions shown in Fig. 1A. Five of the specimens were furnished by St. Louis Precision Casting Co.; three of the gray and ductile irons were cast and processed at Case Western Reserve University (CWRU) and the wrought $\mathrm{H} 13$ material is premium grade steel from a supplier of die steels. The H13 die steel was machined and heat treated at CWRU. The chemical analyses of all of these test materials are listed in Table 1, and the processing details in Table 2. The rapid cooling and tempering of the gray and ductile iron specimens produced at CWRU failed to improve their thermal fatigue resistance compared to these irons in the as cast condition, so these irons are only reported as cast. 
The cast irons and steels produced by St. Louis Precision Castings were cast in nobake sand molds, fairly close to dimensions. Subsequently they were machined to final dimensions after the cast irons were stress-relieved and the cast steels were quenched and tempered. The CWRU alloyed gray and ductile cast irons were cast in sand molds as a 22 inch $(6.35 \mathrm{~cm})$ thick plate and machined. The best results obtained with these irons were in the as cast condition. Heat treating the cast irons by quenching and tempering treatments resulted in poorer thermal fatigue behavior or gross cracking during the immersion cycle, so these treatments were discarded. The wrought H13 steel was from a large block of premium grade $\mathrm{H} 13$ steel received from Latrobe Steel in the annealed condition. This thermal fatigue specimen was machined and heat treated by austenitizing at $1900^{\circ} \mathrm{F}\left(1038^{\circ} \mathrm{C}\right)$, oil quenched and double tempered to 47 Rockwell $\mathrm{C}$ hardness.

\subsection{RESULTS AND DISCUSSION}

The thermal fatigue test results on these different materials are shown in Figures 4 and 5 for the cast iron test pieces and Figures 6 and 7 for the cast and wrought steels with two of the better cast irons added for comparison purposes. Figures 4 and 6 show the average maximum crack lengths and Figures 5 and 7 indicate the total crack area for 
these various materials. The cast irons are plotted separately because they are lower in cost and generally easier to machine to final mold dimensions.

Among the cast irons, the CWRU second gray and CWRU ductile iron compositions had superior thermal fatigue resistance compared to the other gray and ductile irons. The improvement in thermal fatigue behavior is attributed to absence of carbides in the better iron and from a better selection of alloying elements. The higher chromium and molybdenum in the commercial gray iron results in a significant amount of carbides in the matrix of this iron. The presence of the carbides increase the rate of thermal fatigue cracking.

A detailed study of the behavior of the cast irons in Figures 4 and 5 indicates that the best resistance to thermal fatigue was exhibited by the ductile iron cast at CWRU. The behavior of this iron in both Figures 4 and 5 is clearly better than the other materials. The second best material was the second gray iron cast at CWRU, particularly when the total crack area is measured. This iron is superior to the commercial ductile, compacted and gray iron and is also superior to the first alloy gray iron cast at CWRU.

Structural details on the thermal fatigue behavior of this iron are shown in Figures 8,9 and 10 . Figure 8 shows the thermal fatigue pattern with the cracks following the graphite flakes after 1,000 cycles. Figure 9 illustrates the graphite and matrix structure of 
this gray iron and Figure 10 shows a few carbides in the alloy segregated areas of the CWRU gray iron-2. It is noted that a few carbides were present and that a significant difference occurs between the microhardness in the segregated and non-segregated areas. The amount of carbides is significantly less than for the commercial gray iron.

The best performance of the cast irons was provided by the ductile iron produced at CWRU. The general structure of this iron is shown in Figure 11. This iron has a mixed pearlitic and bainitic matrix. The alloy segregates in the lighter etching bainitic areas are indicated by the different sizes of the microhardness indentations. The segregated bainitic structure areas are significantly harder than the pearlitic matrix areas as shown by the microhardness readings in Figure 12. This structure is as cast and segregated to a considerable extent, yet it provides the best thermal fatigue resistance of the cast irons tested.

When the behavior of these cast irons is compared to the steels that were tested, the superiority of the quenched and tempered wrought $\mathrm{H} 13$ premium grade alloy is apparent. The material with the next highest thermal fatigue resistance is the cast and heat treated $\mathrm{H} 13$ alloy followed fairly closely by the CWRU ductile iron specimen referred to above. This ductile iron is actually slightly superior to the cast and heat treated 4140 steel. The poorest thermal fatigue behavior of the selected materials shown in Figures 6 and 7 is for the CWRU gray iron-2 tested in the as cast condition. 


\subsection{CONCLUSIONS}

A thermal fatigue test has been developed for potential mold materials for permanent molds. This test involves immersion in molten aluminum to provide a fluctuation in surface temperature similar to that experienced in permanent molds used for aluminum castings. This test was employed to evaluate the relative thermal fatigue resistance of several different types of mold materials for permanent molding of aluminum. The results indicate that the composition and structure of the mold materials have a considerable effect on their thermal fatigue resistance as tested with this immersion testing. The relative thermal fatigue resistance of the different materials tested also showing their comparative hardness is listed below (best is last).

Mold Material

Commercial Alloy Gray Iron

CWRU Alloy Gray Iron-1

Commercial Compacted Graphite Gray Iron

Commercial Ductile Iron

CWRU Gray Iron-2

Commercial Cast 4140 Steel

CWRU Ductile Iron

Commercial Cast H13 Steel

Commercial Wrought Premium Grade H13

\section{Hardness Before Test}

$210 \mathrm{BHN}$

$217 \mathrm{BHN}$

$235 \mathrm{BHN}$

$273 \mathrm{BHN}$

$228 \mathrm{BHN}$

$404 \mathrm{BHN}$

$245 \mathrm{BHN}$

$437 \mathrm{BHN}$

$448 \mathrm{BHN}$ 


\subsection{REFERENCES}

1. K. Davis, D.A. Braun, J.G. Magny and D. Hui, Choice of Permanent Mold Materials for Iron Casting. AFS Trans. 1989 97: pp. 85-104

2. Y.J. Park and R.B. Gundlach, Thermal Fatigue Resistance of Gray and Compacted Graphite Irons., AFS Trans.1987 95: pp. 267-272.

3. K. Nyamekye, S. Wei and K.M. Martinez, A CAD/CAE Model for Predicting Thermal Fatigue Life of a Permanent Mold. AFS Trans. 1997 105: pp. 557-572.

4. Y.S. Lerner; E.E. Storer, Mold Life in Permanent Mold Casting, Foundry Management \& Technology, March 1998, pp. 32-34.

5. Chiesa, F. and J. Trottier, Mechanics of The Die Coating Application in the Permanent Mould Casting Process. International Journal of Cast Metals Research, 1996. 9: pp. 145-152.

6. Dillingham, J., C.W. Ramsay, and D.R. Askeland, Literature Review for Tenacious Coatings for Aluminum Permanent Mold Casting Process. AFS Transactions, 1996. 162: pp. 1079-1095.

7. Broome, A.J., Mould and Core Coatings and their Application. The British Foundryman, 1980. 73: pp. 96-102.

8. Pischel, R.P., Technical and Economic Impact of Permanent Mold Coating Application. $4^{\text {th }}$ International Conference on Permanent Mold Casting of Aluminum, 1997.

9. Chiesa, F., Permanent Mold Coating Technology. $4^{\text {th }}$ International Conference on Permanent Mold Casting of Aluminum, 1997; 


\section{PART 2}

\section{EVALUATION OF COATINGS FOR PERMANENT MOLDS}

\subsection{INTRODUCTION}

\subsubsection{Purpose and Nature of Coating}

The permanent mold processes comprise all casting techniques using a metallic mold where the liquid metal pressure is close to atmospheric. In order of increasing sophistication, they include the gravity, tilt-pour, low-pressure and counter-pressure processes. For all these processes the mold must be dressed with a ceramic coating before the cavity is filled with liquid aluminum. The main purposes of this coating are the following[1]:

a. To prevent the molten metal from freezing prematurely.

The main contribution of mold coatings is insulation. Because molds are made of a metal with a high thermal conductivity it is necessary to use an insulated coating between the molten stream of metal and the mold itself. This is essential to keep the molten metal from freezing before it reaches all areas of the cavity. 
b. To control the rate and direction of solidification of the casting and, therefore, its soundness and structure.

By varying the thickness of the insulation layer, solidification of the metal at different locations in the cavity can be controlled. This can help ensure a sound casting.

c. To minimize thermal shock to the mold material

Coatings can markedly reduce the temperature gradient at the metal-mold interface during the initial few seconds of casting, thus reducing thermal shock to the mold.

d. To prevent dissolution of the mold into the molten metal and soldering of molten metal to the mold.

Molten aluminum has a high affinity to most other metals. A barrier is necessary between the molten aluminum and the mold to prevent soldering or dissolving. This action is often called erosion or washing. Without some means of preventing it, molds would not last long.

e. To vent air trapped in the mold cavity.

Air in the cavity must be vented before complete filling can occur. A permanent mold has no permeability; air cannot pass through it. Sometimes, grooves are made on the interior of a permanent mold to help evacuate air. If the mold coating is applied on the 
interior of a mold, the textured surface of the mold coating material can also act as tiny channels to evacuate air between the casting and the mold.

\section{f. To lubricate the mold.}

Since aluminum shrinks as it solidifies the casting may grip tightly to certain areas in the mold where draft is minimal. A coating with lubricating properties will allow for release of the casting in these areas[2-8].

\subsubsection{Constituents of the Coating}

In any permanent mold coating, four basic constituents of the coating determine the usage, method of application, method of curing and performance when cast. These include fillers, carrier liquids, suspension agents, and binders. Table 1 lists the composition of some typical coating slurry for permanent molds $[3,9]$.

\subsubsection{Fillers}

The refractory filler determines the effectiveness of the coating in preventing erosion and metal mold reactions. It is selected according to the metal to be cast, the substrate (the mold wall) and the tolerance for possible defects. The refractoriness and the 
sintering point of the material are extremely important. In addition, the filler must have the correct particle size, not react with the metal and withstand the molten metal. Certain fillers may contain compounds that evolve gases during casting and, thus, must be used with discretion. The properties of the most important fillers are listed in Table 2[10]. The thermal conductivity and thermal expansion of the filler affect the expansion of the mold. Thermal conductivity and thermal radiation (white vs. black coating) also play an important part in the rate of substrate heating and in the rate of solidification of the casting. The selected filler should not be too fine. Fine filler particles increase the risk of cracking in the fired coating and increase the proportion of binder required. Table 3 lists the suitability of various fillers for several metal casting alloys[2, 10].

Coke is used mainly in coatings for casting of gray iron and nodular iron. Its properties may vary considerably from salt contamination. The advantages of coke are high melting point, low reactivity and low wettability for almost all cast metals; its major disadvantage is its high porosity, which causes slow drying of the coating. Natured and electrode graphite serves as filler for many coatings. They are highly refractory and are not wetted by many metals. Pure graphite-based coatings are widely used in automotive and intricate iron casting production. Graphite has high thermal conductivity and heat absorption by radiation makes it unattractive for large castings, particularly when the mold substrate must be insulated. 
Other filler constituents include zircon, mica, talc, calcined magnesite and alumina. Zircon is used along with graphite and talc as a major coating refractory. Zircon bonds well and its sintering range begins at temperatures far below its melting point. This probably reduces the stress in the coating, thus reducing spalling and minimizing defects. Mica is a hydrated aluminum silicate with a flake-like structure. When it is heated, water is evolved and its structure is changed causing the material to expand slightly. Mica has a low melting point but can be used for permanent molds of aluminum casting. Talc is a complex hydrated magnesium silicate. As a filler constituent it improves the ability of metal to flow over a coating and provide an insulating effect[10]. Calcined magnesite and aluminum silicates have a high melting point and are used more for higher melting temperature metals than for aluminum.

\subsubsection{Carrier Liquids}

The filler materials are suspended in a carrier liquid, the choice of which is governed by operational conditions including drying and venting. Water is the most important carrier liquid due to its non-toxicity, low price and ease of adjustment of rheological properties. For coatings that must be flame or air dried, alcohol or petroleumbased solvents are used. Occasionally, rapid air-drying solvents are needed; these are normally of the chlorinated type. They are more costly and hazardous than alcohol, but are sometimes used if adequate ventilation is available. 


\subsubsection{Suspension Agents}

Suspension agents are used to prevent settling of the refractory materials. Addition of suspension agent to a mold coating increases the risk of cracking. Bentonites and other clays are often used as suspension agents in aqueous systems. Their advantage lies in good thixotropic behavior and an ability to act as a high temperature binder. The long time needed to stabilize a coating that contains bentonite is, however, a drawback.

\subsubsection{Binders}

Binders are employed to increase the coating strength and adhesion. In aqueous systems, dextrin, sulfite lyes and partly unsaturated carbonic acid esters (such as linseed oil) are used as ambient temperature binders. With dextrin and those sulfite lyes sensitive to bacterial decomposition, antifermentation agents are necessary. Bentonites, other clays, phosphates and sodium silicate serve as high temperature binders in aqueous systems. However, flocculation or peptisation may occur when using the phosphates or sodium silicate with bentonite. In addition to conventional water-soluble binders, dispersion binders similar to systems for the dye industry employed. Alcohol-soluble synthetic resins, such as novalacs, maleinates and ketones, together with natural resins and alcohol-soluble plastics, serve as binders in alcohol systems. The choice of binder is 
made according to its bonding relationship with a given individual refractory. For instance, binders with a good bonding capacity for graphite may not be suitable for zircon[2].

\subsubsection{Permanent Mold Coating Formulation}

Coating formulation for the particular application requirement is critical. Permanent mold coatings of all types must be made from highly consistent and tightly controlled raw materials. Permanent mold coatings can be classified into four basic families: Insulating, Non-insulating, Release and Combination.

Insulating cóatings are produced using combinations of talc, mica, alumina, titania, magnesia etc. As in specific ratios with sodium silicate, these are the most widely used binder systems. The sieve grading, purity and ratio of these materials in the mixture determine their relative insulating capability, durability and surface finish of the resulting coating.

Insulating coatings can also influence the surface finish of the casting due primarily to the morphology differences of the refractory materials used. Talc and mica, for example, are plate-like in form and generally produce a smoother casting surface than 
the more granular materials such as titania and magnesia. Application techniques play a significant role in their behavior. Any coating surface anomalies due to poor application will be directly translated to the casting surface regardless of the capabilities of coating applied. There is also the compromise of durability to be considered. Typically, materials that provide for the best surface finish are also less durable and can be more easily damaged and may wear away more quickly.

Non-insulating coatings have often been incorrectly classified as "chill" coatings. In the strict definition, these coatings cannot be considered chills since they can not increase the rate of heat transfer beyond that of the uncoated metal mold. The rate of heat transfer through the mold material itself can only be increased by air moving through fluid channels within the mold body itself or by cooling applied to the outside of the mold. Non-insulating coatings provide protection for the mold as well as influence the surface finish without diminishing the rate of heat transfer as defined by the mold. Materials such as graphite and boron nitride are typically used for this purpose. Release coatings typically consist of graphite in colloidal of or semi-colloidal form. Their primary function is to lubricate the mold casting interface so that the casting does not stick and can be easily extracted from the mold. Other materials, such as boron nitride, are also sometimes used. Although non-wetting, most lubricating coatings are not insulating. Several compromises may be required in areas where insulation is needed such as the use of multiple coating layers particularly graphite over an insulating coating. 
Combination coatings have also been developed to try and overcome some of the problems of competing and mutually exclusive properties of certain coatings and casting requirements. These include mixtures of lubrication and insulating coatings. However, by their very nature, these combinations compromise both objectives such that they will not insulate as well as a true insulating coating nor will they have all of the release properties of a true release coating[11].

\subsubsection{Properties Required From Mold Coating}

From the foundryman's standpoint, a permanent mold coating will be judged on four characteristics:

1. Adherence to the metallic substrate which controls spalling. This depends on the preparation of the mold surface and on the conditions of deposition and drying of the very first sprayed passes.

2. Mechanical resistance to wear when the surface of the coating is impacted by the viscous shear of the liquid metal during filling.

3. Mechanical resistance to wear by the rubbing action of the casting at ejection.

4. Thermal resistance to heat flow which will provide the required insulation for 
complete filling and directional solidification[1].

\subsubsection{Permanent Mold Coating Application}

The coating can be applied by brushing, dipping, swabbing or spraying. In general, spraying is used more often because of easier control of uniformity of coating thickness. Before the coating is applied, the mold should be clean and free of oil and grease. Portions to be coated should be lightly blasted, most often by aluminum chips or glass beads. Coating should be applied to molds at around $350^{\circ} \mathrm{F}-400^{\circ} \mathrm{F}$. If the molds are too hot, the rapid expanding water vapor front moving away from the mold will cause a phenomenon known "kick back" and mold adhesion will be poor. If the mold is too cold, the coating will dry too slowly which may cause runs and an uneven surface that will show up on the casting. The low temperature and slow drying may also entrap moisture within the coating layer that may cause the coating to crack and flake during use.

A primer coating is generally used to enhance adhesion of the primary coating to the mold surface. This can be a specifically designed coating or can be a diluted version of the main coating. It is important that the coating be applied in a thin layer to ensure maximum durability. Once the primer is applied, the primary coating can be applied at higher concentrations. When building up the main coating layer it is equally important to 
build a succession of thin layers to the desired coating thickness rather than one or two heavy coating layers.

The strength of the coating should be maintained, particularly with insulating coatings. A recent study has demonstrated that for any given coating, its maximum resistance to heat flow is reached when the coating thickness is around 0.008 ". Additional coating thickness does not significantly increase the resistance to heat flow and can contribute to other performance failures. Excessively thick coatings actually wear away more quickly, are more susceptible to cracking, flaking and chipping away from the mold as well as being more likely to produce surface finish problems with the casting.

The performance of any coating is a combination of the coating material and its application method such that the supplied system has maximized the performance characteristic possibilities. The dilution of the coating, the application technique, as well as application parameters such as mold temperature, spray distance, spray angle, spray configuration, all play a role in determining the internal physical porosity characteristics of the coating that actually bonds to the mold. It is the combination of the internal porosity of the coating as well as the thermal characteristic of the coating material itself that determine the degree of insulation. 


\subsubsection{Influence of Dilution and Mold Temperature on Drying Rate}

The spraying of the slurry results in a dry, porous ceramic layer. The porosity and surface texture of the coating will depend on the drying rate after application. This, in turn, depends mainly on the dilution of the coating and the mold temperature. In order to compare slurries with ceramic content of widely differing density, the dilution should be expressed in percent solid content by volume. The solid content of the slurry and its specific gravity depend on the density of the ingredient.

A heat transfer model has been proposed to relate the drying rate of the slurry to the mold temperature. The drying time increases sharply as the coating builds up. The drying speed depends on the initial mold temperature, slurry dilution and coating build up. The results indicate that, for the same initial temperature of the mold, the rate of drying is reduced by a ratio of $5: 1$ when the layer builds up to about $100 \mu \mathrm{m}$. From a practical standpoint, these results can be used to adjust the dilution of a slurry. This can be done using the set of curves of Figure 1. They show the relationship that should be maintained between the mold temperature and slurry dilution for the initial passes (primer coating), and after $100 \mu \mathrm{m}$ build up[1]. 


\subsubsection{Setting and Handling of the Spray Gun}

The physics of the spray gun atomization process and deposition of the coating has been presented in an earlier paper[1]. It was shown that the rate of deposition is mathematically related to the spray gun flow rate, the dilution of the slurry $(\%$ solid by volume) and the surface area of the spray trace. The droplet size is mainly affected by the air pressure, via the air spray velocity and has a very important impact on the covering capability of the coating, as illustrated in Figure 2. It shows that droplets of $200 \mu \mathrm{m}$ will cover hardly $50 \%$ of the mold surface for an average coating deposit of $45 \mu \mathrm{m}$. It is interesting to note that the surface of the coating will be smoother under conditions that produce smaller droplets.

The influence of spray gun handling on the thickness distribution of the coating has also been studied mathematically[1]. Figure 3 shows the coating thickness distribution when the spray gun is rotated about an axis in a more or less sinusoidal motion. It shows that a build up of coating forms on the sides of the mold surface, at the location where the motion is arrested to change its direction. As shown, crossing passes greatly improve the uniformity of the coating thickness from a build up of $60 \%$ above the average thickness, down to around $20 \%$. The situation is greatly improved when the spray is moved from left to right at a constant velocity as shown by the distribution of 
Figure 4. The lower deposit at the top and bottom edge of the plate can be greatly reduced when the side passes are made to overlap[12].

\subsection{EXPERIMENTAL EQUIPMENT AND PROCEDURES}

\subsubsection{Materials}

\subsubsection{Test Specimen}

The thermal fatigue test specimens were produced using wrought $\mathrm{H}-13$ steel, a conventional die-casting die material. It has good resistance to thermal shock and good resistance to softening at elevated temperatures. The composition of $\mathrm{H}-13$ steel is listed in Table $4[13,14]$.

The specimen was machined to a 2 inches square by 7 inches long shape with a 1.5 diameter hole drilled lengthwise in the center of the 2-inch square to depth of 6.5 inches. The specimen was coated on two adjacent sides with various coatings. Thermocouples are located in the two 0.09 " diameter holes that are drilled at 0.1 inch from both sides of the hole at the corner, the depth of the holes is 4.0 inches from the top surface. The temperature variation that occurred during this cyclic immersion of this specimen coated on two of its four sides with the different coating were measured by $\mathrm{K}$ type 
thermocouples inserted in the two corners with and without the coating. The configuration of the specimen is shown in Figure 5.

Table 4. Chemical Composition of H-13 Steel

\begin{tabular}{|l|l|}
\hline Element & Composition in Weight Percent \\
\hline $\mathrm{C}$ & 0.39 \\
\hline $\mathrm{Mn}$ & 035 \\
\hline $\mathrm{Si}$ & 1.00 \\
\hline $\mathrm{Cr}$ & 5.25 \\
\hline $\mathrm{Mo}$ & 1.45 \\
\hline $\mathrm{V}$ & 1.00 \\
\hline $\mathrm{S}$ & $0.005 \max$. \\
\hline $\mathrm{P}$ & $0.025 \max$. \\
\hline
\end{tabular}




\subsubsection{Coatings}

All coatings were obtained from Foseco, Inc., included DYCOTE 8, DYCOTE 11, DYCOTE 34-ESS and DYCOTE R-87. They are different blends of insulating and lubricating refractory materials with a silicate binder and were applied in a concentrated liquid or paste-like form. These coatings are widely used in commercial permanent mold coating.

DYCOTE 34-ESS is a general purpose insulating coating which provides a rough mold surface that enhances metal flow over large flat surfaces and through thin sections, thus reducing misruns and cold shuts.

DYCOTE 8 is a highly insulating and very durable coating specifically recommended for use on gates and risers at a relatively heavy consistency. For general purpose or primer applications, it should be diluted at higher ratio.

DYCOTE R-87 is an extremely durable permanent mold coating. It is often used as a base coat, providing excellent adhesion between the topcoat and the mold. It also provides a very smooth surface finish when used as a topcoat. Because of its excellent adhesion properties, DYCOTE R-87 can also be used to coat foundry tools and lowpressure stalk tubes. 
DYCOTE 11 is a graphic lubricating coating that works well on ingot molds, pigging machines, and ejector pins. It may be used on top of an insulating coating in deep-draw or high-wear areas to promote release and reduce coating wear.

All of the DYCOTE permanent mold coatings are easily diluted with water. In general, the coatings diluted for priming purposes are diluted $2-3$ times more than for primary application. The recommended dilution ratio for primer coatings and main coatings and approximate compositions of four DYCOTE coatings are shown in Table $5[9,15]$

\subsubsection{Equipment}

\subsubsection{Thermal Fatigue Immersion Testing Unit}

The thermal fatigue immersion-testing unit is shown schematically in Figure 6. The semi coated metal specimen, two adjacent corners - coated and uncoated, was cycled between immersion in molten 356 aluminum alloy and cooling in ambient air. The aluminum alloy was maintained in a gas fired crucible furnace at an average temperature of $1350^{\circ} \mathrm{F}$ which was monitored by chromel-alumel thermocouple. The cycle immersion 
time was 12 seconds and was followed by a 24 second cooling time. The specimen is internally water cooled at a rate of 1.5 gallon per minute.

\subsubsection{EIcometer 355}

This is the gauge used to test the thickness of coating. It has two parts. The host instrument, which is a portable, hand held unit powered by three AA cells collects measurements data; A probe module, which is available for coating thickness measurement on both ferrous $(\mathrm{F})$ and non-ferrous $(\mathrm{N})$ metals and further modules will be added to the range as the product develops. The probe type used for this test is F4 standard. Measuring range is $0-10 \mathrm{mil}$. The accuracy is $\pm 1 \%$ or $\pm 0.04 \mathrm{mil}$.

The instrument uses Liquid Crystal Display, so the ambient temperature could not be above $50{ }^{\circ} \mathrm{C}$. The sketch of Elcometer 355 is shown on Figure 7.

\subsubsection{WorkBench PC}

Temperatures at each of the two testing locations were continuously monitored, using "WorkBench PC" (v2.3.1, Strawberry Tree Inc.). It is a data acquisition, process control, and analysis software which takes full advantage of the features and the graphical 
interface. Data was collected at one-second interval and eventually transferred to a standard spreadsheet for subsequent analysis.

\subsubsection{Procedures}

\subsubsection{Sample Preparation}

The surface of the metal sample was first cleaned with sandpaper to remove any old coating, grease and oil completely. Then the specimen was heated to $325-375$ ${ }^{\circ} \mathrm{F}[16]$. The coatings were sprayed onto the two adjacent corners of metal sample with spray gun. The pressure of compressed air is $80-100 \mathrm{psi}$ and the spraying distance is about 7 inches[17]. The primer coating was sprayed on the two adjacent corners first until the dark color of metal sample began to disappear. The thickness of primer coating was less than 0.001 ". Then the specimen was heated up to $325-375^{\circ} \mathrm{F}$ again, and the primary coating on the same two adjacent corners. The total thickness of the coatings was about 0.008 ". After applying the coating on the metal specimen, the final thickness of the coating was measured and a picture was taken of the coated specimen. Finally the coating was dried in the oven for 20 hours at $250^{\circ} \mathrm{F}$. The coating dilution ratios and coating thickness are shown in Table 6. 


\subsubsection{Testing}

\section{Coating thickness testing}

Before the sample was cyclically dipped into the molten metal, the thickness of coatings was tested on the top part of the sample, which could not be immersed into the liquid metal. Five locations on each side were checked to provide an average value. After the sample was cycled, the thickness of the coating was tested on the whole side. Each side was tested on three lines - the centerline and the two edge lines, at about 0.3 " from the edge of the sample. 10 points were tested on each line to obtain an average value.

\section{Temperature difference testing}

The specimen with dried coating was immersed in 365 molten metal for 100 cycles. The temperature variations that occurred during this cyclic immersion of the specimen coated on two of its four sides were measured by thermocouples on the corners with and without the coating. The temperature variations on the corners of this specimen were monitored with WorkBench PC and the data were recorded for the 1-10 dips, $11-$ 20 dips, the $61-70$ dips and $91-100$ dips. The circulating water temperature fluctuations were also measured immediately after the water exited from the specimen. 
The temperatures of the coated and uncoated corners during the immersion cycles were recorded as shown for each of the four coatings in the temperature versus time graphs in the Appendix. After the specimens had been cyclically immersed the surface condition of the coatings were measured and photographed. The pictures of the coated specimen before and after testing are shown in the following section.

\section{Coating adhesion testing}

The adhesion of the coating to the metallic substrate was also tested according to ASTM D4541 - 95, “ Standard Test Method for Pull-Off Strength of Coatings Using Portable Adhesion Testers"”. The sample was tested after being cyclically immersed. Two points were tested on each side.

\section{Roughness testing of the coating}

In order to test the roughness of the coatings, the sample substrate was changed from the metal specimen to a graphite thin plate. Different coatings were sprayed on the graphite plates. Coating dilution and spraying processing were all the same as for the metal specimen. The coated graphite plates were fractured into several pieces and the cross-section of the broken graphite pieces with the coating on the sample was examined with SEM. Three photographs for each coating were taken at a magnification of $100 \times$. 
The height difference between two adjacent high and low points was measured from the photographs. Thirty pairs of points were measured to test the height difference, and obtain an average value for the roughness of the coating.

\subsection{RESULTS AND DISCUSSION}

The data on the cyclic temperature variations was averaged over 10 dips into molten aluminum of the immersion test, each including the 1-10 dips, 11-20 dips, 51-60 dips and 91-100 dips. The results are listed in various tables and the actual temperature fluctuations with time are shown in the Appendix.

\subsubsection{Thermal analysis of uncoated corner of sample}

Table 7 shows the temperature difference of the uncoated corner during immersion. The average maximum temperatures for different cyclic periods are almost stable, or do not change at high dipping cycles. The molten metal temperature is the only factor that determines the maximum temperature of the uncoated corner. The higher the molten metal temperature, the higher the maximum average temperature of the uncoated corner. For the average minimum temperature, the data are close to each other for the 
various dipping cycles. For the coating Dycote 8, Dycote 34-ESS and Dycote R87, these values are all close to $250^{\circ} \mathrm{F}$. This behavior occurs because the minimum temperature of the uncoated corner is just influenced by ambient temperature, and the ambient temperature is stable. The data in Table 7 shows little change for the minimum and maximum temperatures of the uncoated corner, as the dipping cycles increase. As expected only a small change occurs in the difference between maximum and minimum temperature. For the minimum temperature of the coated corner, the temperature is also kept increasing with the minimum temperature of the uncoated corner. This follows the same trend as the average maximum temperature. The higher the molten metal temperature, the larger the difference in the temperature.

\subsubsection{Thermal analysis of coated corner of sample}

The average temperature of the coated corner, as shown in Table 8 , is considerably different. All of the temperature values are lower for the coated corner because of the reduced heat transfer. The average maximum temperature of the coated corner keeps increasing with increasing number of dipping cycles for all four coatings. This behavior is illustrated in Figure 8. It is also noted that the temperature of the molten metal temperature did not have a marked influence on the temperatures of coated corner. Two factors influence the maximum temperature of coated corner as the dipping cycles are 
increased. First the increasing temperature of the specimen as the number of cycle increases. Second, the effect of increasing cycles on the thickness of the coating influence temperature. When the coated specimen was cyclically dipped into the molten metal, the liquid metal wears off some of the coating. The thinner the coating layer, the higher the heat that is transferred from the molten metal. So the maximum temperature of the coated corner can increase with increasing cycles. As noted in Table 8, the final thickness of all four coatings is lower than the original thickness. And the loss in thickness increases with the number of dipping cycles. Because the temperature of the other parts of the specimen increase with increasing number of cycles, the minimum temperature of coated corner increases with time. In Table 8, the temperature of the coated corner is influenced by the insulation of the coating. The greatest difference between the maximum and minimum temperatures was attained for the wash with the highest heat transfer. The least difference in temperature was obtained that the wash with the best insulating effect.

Figure 9 shows the average difference between maximum and minimum cycle temperature in coated corner. This plot indicates that coating Dycote 8 is the best insulator followed by Dycote 34-ESS as the second most effective insulator. The Dycote R87 is the third best insulator. The lowest insulating ability and the highest heat transfer of the four coatings occurs for Dycote 11. 
The average maximum temperature difference between the coated and uncoated corner also relates to the insulating capability of the different coatings. The larger the difference, the more effective the coating was as an insulator. The average maximum temperature differences between coated and uncoated corner for the four coatings have been plotted in Figure10. The results are the inverse of those obtained in Figure 9 and show the same relative behavior in insulating ability for the castings. Figures 9 and 10 indicate that the relative insulating ability is Dycote 8, Dycote 34-ESS, Dycote R87 and Dycote 11 for the best to lowest insulation.

\subsubsection{Calculation of heat transfer coefficient of different coatings}

When the sample is cyclically immersed into the molten metal, the heat transferred from molten metal to sample $\left(\mathrm{Q}_{\mathrm{m} \mathrm{s}}\right)$ equals to the summation of the heat transferred from sample to water $\left(Q_{s w}\right)$, lost in the air from sample $\left(Q_{s a}\right)$, and remained in the sample $\left(\mathrm{Q}_{\text {remain }}\right)$. This is shown by:

$$
\begin{array}{ll}
\mathbf{Q}_{\mathrm{m} \text { s }}=\mathbf{Q}_{\text {s }}+\mathbf{Q}_{\text {remain }}+\mathbf{Q}_{\text {s a }} & \ldots \ldots \ldots \ldots \text { (1) } \\
\text { where } & \text { s: sample } \\
& \text { w: water } \\
& \text { m: metal } \\
& \text { a: air }
\end{array}
$$

The heat flow into the water is:

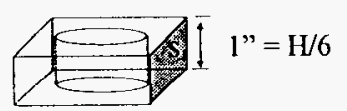

$$
\mathrm{q}_{\mathrm{s} w}={ }_{\mathrm{w}} \times \mathrm{C}_{\mathrm{pw}} \times \mathrm{r} \times \mathrm{T}_{\mathrm{w}}
$$

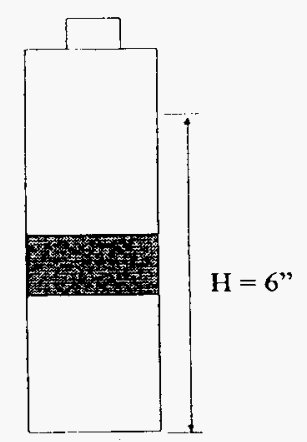


where $\quad r$ : volume flow rate

$$
\mathrm{T}_{\mathrm{w}}=\mathrm{T}_{\mathrm{m}}-\mathrm{T}_{\mathrm{i}}
$$

$\mathrm{T}_{\mathrm{m}}$ : measured water temperature

$\mathrm{T}_{\mathrm{i}}$ : initial water temperature

The heat transferred to water is:

$$
\begin{aligned}
\mathrm{Q}_{\mathrm{s} w} & ={ }_{\mathrm{w}} \times \mathrm{C}_{\mathrm{pw}} \times \int_{t 1}^{\prime 2} \Delta \mathrm{T}_{\mathrm{w}} \cdot \gamma d t \\
& ={ }_{\mathrm{w}} \times \mathrm{C}_{\mathrm{pw}} \times \mathrm{T}_{\mathrm{w}} \times \int_{t 1}^{12} \gamma d t \\
& ={ }_{\mathrm{w}} \times \mathrm{C}_{\mathrm{pw}} \times \mathrm{T}_{\mathrm{w}} \times\left(\frac{1}{6} \mathrm{~V}\right) \\
& =\mathrm{C}_{\mathrm{pw}} \times \mathrm{T}_{\mathrm{w}} \times\left(\frac{1}{6} \mathrm{~W}\right) \\
\therefore \mathrm{Q}_{\mathrm{s} w} & =\mathrm{C}_{\mathrm{pw}} \times \mathrm{T}_{\mathrm{w}} \times\left(\frac{1}{6} \mathrm{~W}\right) \ldots .
\end{aligned}
$$

where $\quad \mathrm{V}$ : volume of water flowing out in one cycle

$$
\text { W: weight of water flowing out in one cycle }
$$

For one cycle, in the ideal condition, $\mathrm{Q}_{\text {remain }}$ equals to zero.

The heat flow $\mathrm{q}=\mathrm{h} \times \mathrm{T}$

$$
\begin{aligned}
& \mathrm{Q}_{\mathrm{ms}}=4 \mathrm{~h}_{1} \mathrm{~S}_{1} \int_{\delta}\left[\int_{t 1}^{t 2}\left(T_{m}-T_{s}\right) d t\right] \cdot d s \\
& \mathrm{Q}_{\mathrm{s} \text { a }}=4 \mathrm{~h}_{2} \mathrm{~S}_{\mathrm{I}} \int_{S}\left[\int_{t 2}^{t 3}\left(T_{s}-T_{a}\right) d t\right] \cdot d s
\end{aligned}
$$

where $\quad \mathrm{T}_{\mathrm{a}}$ : air temperature

$\mathrm{s}_{1}$ : side area of the sample $\mathrm{s}_{1}=1 \times 2=2 \mathrm{in}^{2}$

$\mathrm{h}_{1}$ : heat coefficient between molten metal and sample

$\mathrm{h}_{2}$ : heat coefficient between sample and air 
Because the heat transfer coefficient between the molten metal and sample is much bigger than the heat transfer coefficient between sample and air, it is assumed that the heat coefficient between sample and air is zero. It is also assumed that the temperature in any position of the sample surface is the same.

$$
\begin{aligned}
\mathrm{Q}_{\mathrm{ms}} & =4 \mathrm{~h}_{1} \times \mathrm{S}_{1} \times \int_{1}^{12}\left(T_{m}-T s\right) d t \\
& =4 \mathrm{~h}_{1} \times \mathrm{S}_{1} \times \mathrm{A}_{1} \\
\mathrm{Q}_{\mathrm{m} \mathrm{s}} & =4 \mathrm{~h}_{1} \times \mathrm{S}_{1} \times \mathrm{A}_{1} \ldots \ldots \ldots \ldots . .
\end{aligned}
$$

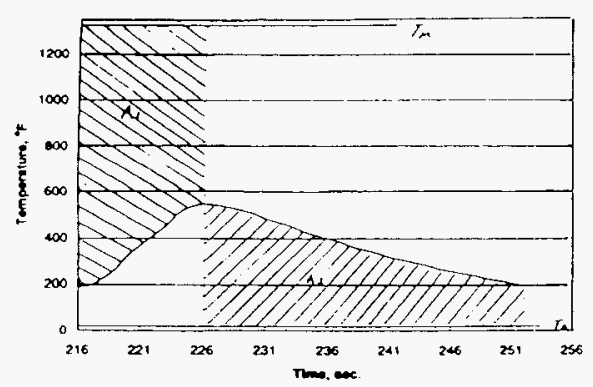

For one cycle, combine the equation (1), (2) and (3), get: $Q_{m s}=Q_{s w}$

$$
\begin{aligned}
& 4 \mathrm{~h}_{1} \times \mathrm{S}_{1} \times \mathrm{A}_{1}=\mathrm{C}_{\mathrm{pw}} \times \mathrm{T}_{\mathrm{w}} \times\left(\frac{1}{6} \mathrm{~W}\right) \\
& \therefore \mathrm{h}_{1}=\frac{\mathrm{C}_{\mathrm{pw}} \times \Delta \mathrm{T}_{\mathrm{w}} \times\left(\frac{1}{6} \mathrm{~W}\right)}{4 \mathrm{~S}_{\mathrm{l}} \mathrm{A}_{\mathrm{l}}}
\end{aligned}
$$

\section{Parameter}

$$
\begin{aligned}
& \mathrm{C}_{\mathrm{pw}}=4.1784 \mathrm{~kJ} / \mathrm{kg}^{\circ} \mathrm{C} \\
& \mathrm{S}_{1}=\mathrm{S}_{2}=1 \times 2 \mathrm{in}^{2}=2 \mathrm{in}^{2}=6.45 \times 10^{-4} \mathrm{~m}^{2} \\
& \mathrm{~S}=4 \mathrm{~S}_{1}=8 \mathrm{in}^{2}=2.58 \times 10^{-3} \mathrm{~m}^{2} \\
& \mathrm{~W}=\frac{1}{5} \times 40 \mathrm{lb}=8 \mathrm{lb}=3.628 \mathrm{~kg} \\
& \mathrm{~T}_{\mathrm{w}}=(29-20)^{\circ} \mathrm{C}=9{ }^{\circ} \mathrm{C}
\end{aligned}
$$

\section{Coating Dycote 8}

$$
\mathrm{A}_{1}=6000^{\circ} \mathrm{C} \cdot \mathrm{sec}
$$




$$
\begin{aligned}
\mathrm{h}_{1} & =1.468 \mathrm{KJ} / \mathrm{m}^{2}{ }^{\circ} \mathrm{C} \cdot \mathrm{sec} \\
& =1468 \mathrm{~W} / \mathrm{m}^{2} \cdot \mathrm{K}
\end{aligned}
$$

\section{Coating 34-ESS}

$$
\begin{aligned}
\mathrm{A}_{1} & =5725^{\circ} \mathrm{C} \cdot \mathrm{sec} \\
\mathrm{h}_{1} & =1.539 \mathrm{KJ} / \mathrm{m}^{2}{ }^{\circ} \mathrm{C} \cdot \mathrm{sec} \\
& =1539 \mathrm{~W} / \mathrm{m}^{2} \cdot \mathrm{K}
\end{aligned}
$$

\section{Coating R87}

$$
\begin{aligned}
\mathrm{A}_{1} & =5540{ }^{\circ} \mathrm{C} \cdot \mathrm{sec} \\
\mathrm{h}_{1} & =1.591 \mathrm{KJ} / \mathrm{m}^{2}{ }^{\circ} \mathrm{C} \cdot \mathrm{sec} \\
& =1591 \mathrm{~W} / \mathrm{m}^{2} \cdot \mathrm{K}
\end{aligned}
$$

\section{Coating Dycote11}

$$
\begin{aligned}
\mathrm{A}_{1} & =5395{ }^{\circ} \mathrm{C} \cdot \mathrm{sec} \\
\mathrm{h}_{1} & =1.634 \mathrm{KJ} / \mathrm{m}^{2}{ }^{\circ} \mathrm{C} \cdot \mathrm{sec} \\
& =1634 \mathrm{~W} / \mathrm{m}^{2} \cdot \mathrm{K}
\end{aligned}
$$

\begin{tabular}{|l|l|l|}
\hline Ranking & Coating & Thermal conductivity $\left(\mathrm{W} / \mathrm{m}^{2} \cdot \mathrm{K}\right)$ \\
\hline 1 & Dycote 11 & 1634 \\
\hline
\end{tabular}




\begin{tabular}{|l|l|l|}
\hline 2 & R87 & 1591 \\
\hline 3 & Dycote 34-ESS & 1539 \\
\hline 4 & Dycote8 & 1468 \\
\hline
\end{tabular}

The values of thermal conductivity measured in the study depend on the method used for measurement. The immersed specimen has heat transferred at the two adjacent coated and uncoated corners as well as to the water-cooling. This arrangement results in higher values of heat coefficient than determined from a single surface. [12]

The insulating effect of a coating is decided by four factors: thermal resistance to heat flow, adherence to the metal substrate, mechanical resistance to wear by the metal flow and the surface roughness of the coating. The thermal resistance of a coating is not only affected by the physical properties of the ceramic powders, but also by the volume percentage of porosity in the coating. The wear resistance of the coating is influenced by the microstructure of the coating. Coatings consisting of powders of ground particles, such as iron oxide, zircon and magnesium oxide, have higher wear resistance than the coatings with a more flake-like structure, such as mica and talc. It must be emphasized that the texture and surface morphology of a coating have as much of an effect on its working properties as the mineralogical nature of the ceramic content. The coarser 
particles are intended to impart surface roughness and hence a higher insulating capability to the coating.

\subsubsection{Analysis of Dycote 8}

Coating Dycote 8 is a high insulating coating. The appearance of the Dycote 8 is shown in Figure 11. The figure highlights the plate-like morphology, especially at higher magnification. From the EDS, the chemical analysis of this coating is shown in Figure 12. The chemical contents of the three spots indicate that they are soft and good insulating minerals such as mica $\mathrm{Al}_{2} \mathrm{~K}\left(\mathrm{Si}_{1.5} \mathrm{Al}_{0.5} \mathrm{O}_{5}\right)_{2}(\mathrm{OH})_{2}$ and talc $\mathrm{Mg}_{3} \mathrm{Si}_{4} \mathrm{O}_{10}(\mathrm{OH})_{2}$. Figure 11 exhibits a high porosity content in the coating. Both the high insulating mineral and the porosity content results in a high thermal resistance for Dycote 8. The coating also contains some harder and more resistant species such as magnesia from the analysis of the Figure 12. In Figure 13, the overview EDS testing for coating Dycote 8 is noted that, a small amount of titanium is present in the coating. Figure14 also shows there is ground oxide particles in Dycote 8. EDS testing indicates the presence of considerable $\mathrm{Na}$ and $\mathrm{Si}$ elements everywhere in Dycote 8 form the sodium silicate binder. These factors explain the high wear resistance and very low thickness loss for Dycote 8 when it was cyclically dipped into the molten aluminum. Figure $15 \& 17$ show a rough surface texture for the Dycote 8 before cyclic running. The adhesion of this coating, as shown in Figure 16, after 
100 cycles is good because it sticks to the metal substrate well with no spalling area and cracks.

Adhesion testing demonstrate the high adhesion of coating Dycote 8 is high. This combination of thermal resistance, wear resistance, roughness and adhesion of the coating provide the good insulating properties of Dycote 8 .

The roughness of Dycote 8 is shown in Figure 17. This coating was prepared on top of a graphite plate that was subsequently fractured. The roughness was measured to be $50 \mu \mathrm{m}$. And the coating was totally $157 \mu \mathrm{m}$ thick.

\subsubsection{Analysis of Dycote 34-ESS}

Dycote 34-ESS is also an insulating coating. From Table 5, the composition of Dycote 34-ESS indicates the presence of the insulating ceramic powders - talc and mica. Figure 18 indicates that the structure of coating Dycote 34-ESS is mainly a plate-like morphology. This structure also produces a high thermal resistance. However compared to Dycote 8 , the grain size of 34-ESS is smaller, and the coating is denser than Dycote 8 with less porosity. Even though the main chemical contents are similar for the two coatings, the thermal resistance of Dycote 34-ESS is lower than for Dycote 8. The EDS 
analysis in Figure 19 shows the presence of small content of magnesium Iron and titanium oxides are present in Dycote 34-ESS and the binder content is lower. The reduced amount of oxide powders and binder content makes results in a lower wear resistant of Dycote 34-ESS compared to Dycote 8. Figure 21 shows that the coating has been washed off completely in some area of the sample after 100 immersion cycles in molten metal. The reduced wear resistance of the coating lowers the thermal effect of Dycote 34-ESS compare to Dycote 8. Although the roughness of Dycote 34-ESS is the highest in the four coatings, as shown in Figure $20 \& 22$, the insulating effect is lower. Good adhesion is also indicated for 34-ESS.

The roughness of Dycote 34-ESS is shown in Figure 22. This roughness was measured to be $63 \mu \mathrm{m}$, and the coating was $221 \mu \mathrm{m}$ in total thickness.

\subsubsection{Analysis of Dycote R87}

Dycote R87 is a coating used as a base or topcoat. Its chemical content is just hard zircon and iron oxide. This is shown in both Table 5 and the EDS testing in Figure 24. EDS testing also indicates that the binder content in this coating is high. This coating does not have any insulating materials like mica and talc, which have flake-like structure. The appearance of the coating is shown in Figure 23. The coating is relatively dense 
though fluffy in appearance. Individual oxide particles are visible at higher magnification. The coating surface appears to have a much lower porosity than the previous two coatings discussed. With its structure and composition, Dycote R87 does not have a high thermal resistance but does have excellent wear resistance. The high wear resistant capability is indicated by a coating thickness, after 100 cycles, that is still has $49 \%$ of the original coating. Figure 26 demonstrate that the coating surface is still smooth and uniform after immersion testing. The surface roughness of coating Dycote R87 is very fine, with the lowest value of $20 \mu \mathrm{m}$, as shown in Figure $25 \& 27$. The adhesion of the coating is not as good as the earlier two coatings. The photo at 150 magnification shows the small cracks on the coating. After cyclic immersion, spalled area or large cracks on the coating surface were not present, as shown in Figure 26. This indicates fairly good adhesion and is confirmed by the adhesion test of Dycote R87. Dycote R87 is a good top or base coating. But its reduced insulating effect does not provide a high insulating coating.

The roughness of Dycote R87 was measured on the fractured section of graphite with roughness of coating is $20 \mu \mathrm{m}$. And total coating was $225 \mu \mathrm{m}$ thick. This roughness is illustrated in Figure 27. 


\subsubsection{Analysis of Dycote 11}

Dycote 11 is a graphite lubricating coating. It is usually used to promote release and reduce coating wear. Figure 28 shows the morphology of the Dycote 11 coating. From the shots taken at low magnification, it shows that the particle size is fine compared with other three coatings. The roughness test from cross-section photograph in Figure 30 also shows the roughness value to be the smallest, at $31 \mu \mathrm{m}$. While considerable porosity is present in the coating, the density is fairly high. The picture at higher magnification in Figure 28 shows the flake-like microstructure of the graphite particle. The insulation produced by this coating is low and the wear resistance is very low. There is only $25 \%$ coating thickness left after the immersion testing. The EDS result in Figure 29 indicates that this coating is nearly all carbon with only a small amount of sodium silicate binder. This explains the low durability of Dycote 11 although the adhesion is as good as other three coatings. The flake-like microstructure of graphite in Dycote 11 and low binder content provide very good lubricating behavior.

The roughness of the Dycote 11 coating, as shown from a coated fractured graphite plate, was measured as $31 \mu \mathrm{m}$, and total thickness was $122 \mu \mathrm{m}$. The appearance of this roughness is shown in Figure 30. 


\subsection{CONCLUSIONS}

A group of four commercial washes were applied to the immersion type specimen normally employed to evaluate the thermal fatigue resistance of mold materials. The washes were applied to two adjacent corners on the thermal fatigue specimen using a controlled spraying technique that applied both a primer and final coating. The normal thickness of the coating before testing was $200 \mu \mathrm{m}$. Two thermo-couples were placed in the opposite corners of the thermal fatigue specimen. One thermo-couple was located between two adjacent coated sides of the specimen and the other between the two uncoated sides. As the specimen was immersed in the molten aluminum, the thermocouple responses were recorded. The result of the temperature fluctuations was employed to evaluate the thermal resistance of the coatings and their durability after a series of immersions in molten aluminum.

The four coatings tested included:

a. Dycote 8 , a highly insulating coating comprised primarily of talc, mica and metal oxide. This wash exhibited the best insulating ability and proved to be very durable upon repeated testing.

b. Another insulating wash Dycote 34-ESS was tested. Its flake-like structure comprised mica and talc with some less binder than Dycote 8 . This is a good 
insulating wash although somewhat less than the Dycote 8 . The coating wore off gradually during the test.

c. The Dycote R87 wash comprises of zircon and some iron oxide bonded with considerable sodium silicate. This wash has relatively poor insulating capability but good durability.

d. Dycote 11 is a primarily graphite wash with some sodium silicate binder. The insulating and wear resistance was poor but it would provide a lubricating effect.

The insulating quality of the washes was evaluated by comparing the temperature difference between the coated and uncoated corners as the specimen was cycled. The minimum temperature difference between the high and low temperature on the coated corner and maximum temperature difference between the coated and uncoated corners indicates the insulating qualities. The durability and wear resistance of the coatings were determined by the loss of insulating ability and the adherence of the coatings during thermal cycling.

The as-deposited roughness of the coatings applied to a graphite plate that was later fractured varies from 20 to $63 \mu \mathrm{m}$ and the total coating thickness from 122 to $221 \mu \mathrm{m}$ 


\subsection{REFERENCES}

1. Chiesa, F. and J. Trottier, Mechanics of The Die Coating Application in The Permanent Mould Casting Process. International Journal of Cast Metals Research, 1996. 9: p. 145-152.

2. Nyamekye, K., et al., A Review of Permanent Mold Coatings and Their Effect on Heat Transfer in The Mold. ASF Transaction, 1994. 143: p. 869-876.

3. Handbook, M., ASM International, , vol 15(9th edition): p. p 281.

4. Chiesa, F., Measurement of the Thermal Conductance at the Mold / Metal Interface of Permantent Molds. AFS Transactions, 1990. vol 98: p. pp 193 - 200.

5. V. Mohan, R.N.S., Prediction of Solidification Time of Iron Castings in Coated Cast Iron Molds. AFS Transactions, 1982. vol 90: p. pp 435 - 444.

6. Ohtsuka, Y., Application of a Computer Simulation system to aluminum Permanent Mold castings. AFS Transactions, 1982. vol 190: p. pp 635 - 646.

7. Nehru, K.V.K., Influence of Insulating Mold Coatings on the Solidification of Copper castings in Metallic Molds and Thermal Behavior of Molds. AFS Cast Metals Research Journal, 1974: p. pp 111 - 116.

8. Chijiiwa, K., Research on the Behavior of Cast Metals in Mold Cavities by the Color Method. Cast Metals Research Journal, 1967: p. pp 113 - 119.

9. Dillingham, J., C.W. Ramsay, and D.R. Askeland, Literature Review for Tenacious Coatings for Aluminum Permanent Mold Casting Process. AFS Transactions, 1996. 162: p. 1079-1095.

10. Broome, A.J., Mould and Core Coatings and their Application. The British Foundryman, 1980. vol 73: p. pp $96-102$.

11. Pischel, R.P., Technical and Economic Impact of Permanent Mold Coating Application. 4th International Conference on Permanent Mold Casting of Aluminum, 1997.

12. Chiesa, F., Permanent Mold Coating Technology. 4th International Conference on Permanent Mold Casting of Aluminum, 1997. 


\section{TABLES AND FIGURES FOR PART 1}

\section{MOLD MATERIALS}




\begin{tabular}{|c|c|c|c|c|c|c|c|c|c|}
\hline$\sum_{1}^{\infty}$ & $\begin{array}{l} \\
\end{array}$ & छ్ & ठ্. & $\stackrel{8}{8}$ & 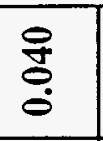 & $\begin{array}{l}8 \\
\stackrel{8}{0} \\
0\end{array}$ & 兽 & $\begin{array}{l}0 \\
0 \\
0\end{array}$ & ठ̊. \\
\hline $1>$ & $\stackrel{\widetilde{\delta}}{\circ}$ & $\mathbb{z}$ & ڤ్ & $\stackrel{\infty}{\stackrel{0}{0}}$ & 今. & ఫ్రి & $\stackrel{0}{0}$ & 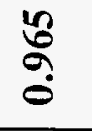 & ஃ̊ \\
\hline ঊ & 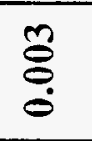 & $\frac{\pi}{z}$ & $\stackrel{8}{8}$ & $\stackrel{n}{0}$ & $\begin{array}{l}m \\
\stackrel{m}{0} \\
\dot{0}\end{array}$ & $\begin{array}{l} \\
\\
0\end{array}$ & $\begin{array}{l} \\
\stackrel{0}{0} \\
0\end{array}$ & $\stackrel{0}{\circ}$ & ठั \\
\hline$|i \mathbf{z}|$ & 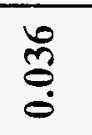 & $\vec{z}$ & ठ్. & 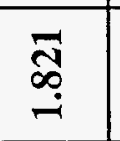 & 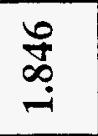 & $\begin{array}{l}\text { Oे } \\
0 \\
0\end{array}$ & $\begin{array}{l}0 \\
0 \\
0 \\
0\end{array}$ & $\begin{array}{l}0 \\
\stackrel{0}{0} \\
0 \\
0\end{array}$ & $\frac{8}{6}$ \\
\hline ปี & $\begin{array}{l}\overrightarrow{0} \\
\stackrel{0}{0}\end{array}$ & $\stackrel{\mathscr{0}}{\stackrel{0}{0}}$ & 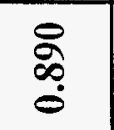 & $\stackrel{\infty}{0}$ & $\begin{array}{l}0 \\
\text { 量 } \\
\end{array}$ & 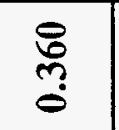 & 商 & $\stackrel{\mathbb{\infty}}{\stackrel{0}{0}}$ & $\stackrel{8}{8}$ \\
\hline$\stackrel{Q}{2}$ & 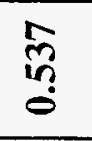 & $\underset{9}{9}$ & $\begin{array}{l}8 \\
\\
0\end{array}$ & $\begin{array}{l}\mathbb{8} \\
\stackrel{8}{0}\end{array}$ & \begin{tabular}{l}
$\infty$ \\
ป̀ \\
\hdashline
\end{tabular} & 。্户 & $\stackrel{n}{\mathscr{C}}$ & $\stackrel{\Im}{7}$ & 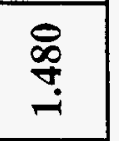 \\
\hline ت & $\stackrel{\overbrace{}}{\overparen{\overbrace{}}}$ & $\stackrel{\text { : }}{\mathrm{d}}$ & $\stackrel{\text { ஸे }}{\stackrel{0}{\circ}}$ & $\stackrel{0}{\dot{g}}$ & 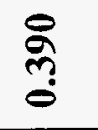 & ڤ్స్ & $\stackrel{\substack{\infty \\
\infty}}{\stackrel{0}{\infty}}$ & $\begin{array}{l}\stackrel{\tilde{F}}{\infty} \\
\stackrel{+}{+}\end{array}$ & 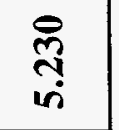 \\
\hline$\infty$ & $\stackrel{5}{0}$ & $\stackrel{8}{0}$ & $\stackrel{\overrightarrow{0}}{\circ}$ & हे & $\stackrel{\infty}{0}$ & $\stackrel{\overrightarrow{0}}{0}$ & $\stackrel{\overrightarrow{0}}{0}$ & $\stackrel{\infty}{\circ}$ & ठิ \\
\hline $\mid$ & 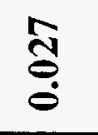 & $\vec{\square}$ & $\stackrel{\overparen{త ్}}{3}$ & $\stackrel{\substack{0 \\
0}}{8}$ & స్త్రి & $\stackrel{\mathscr{0}}{\stackrel{0}{0}}$ & $\stackrel{1}{0}$ & 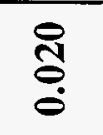 & 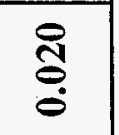 \\
\hline s & 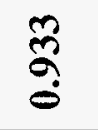 & $\stackrel{0}{\stackrel{9}{0}}$ & 总 & $\stackrel{\overrightarrow{7 n}}{8}$ & 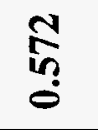 & 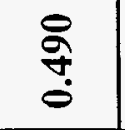 & $\stackrel{\vec{\overbrace{}}}{\stackrel{0}{\delta}}$ & $\stackrel{\substack{0 \\
:}}{\stackrel{n}{0}}$ & 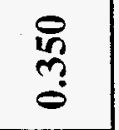 \\
\hline |ळ & 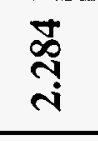 & $\underset{\substack{n \\
i}}{2}$ & $\stackrel{8}{8}$ & 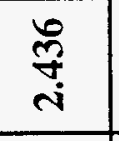 & 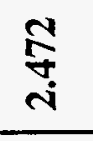 & 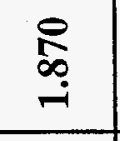 & $\stackrel{9}{7}$ & $\stackrel{n}{8}$ & $\stackrel{?}{\stackrel{2}{2}}$ \\
\hline 0 & तี & $\stackrel{n}{m}$ & $\stackrel{m}{m}$ & $\stackrel{3}{3}$ & $\begin{array}{l}\vec{b} \\
\dot{n}\end{array}$ & in & $\stackrel{\infty}{0}$ & $\stackrel{\infty}{0}$ & $\stackrel{9}{9}$ \\
\hline 莗 & $\sum_{0}^{0}$ & & & $\begin{array}{l}8 \\
8 \\
5 \\
\end{array}$ & $\begin{array}{l}\infty \\
\infty \\
\hbar\end{array}$ & & & & \\
\hline$\left|\begin{array}{c}\frac{\pi}{n} \\
\frac{\vec{n}}{\pi} \\
\frac{\pi}{2}\end{array}\right|$ & 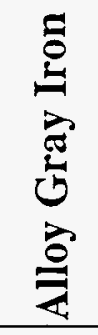 & 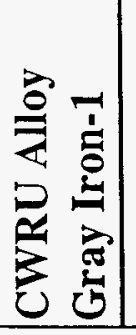 & 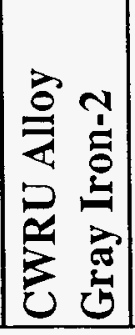 & 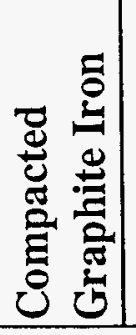 & 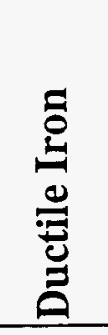 & 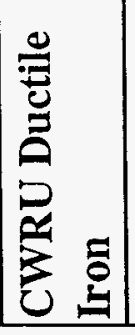 & 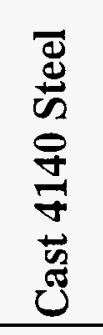 & 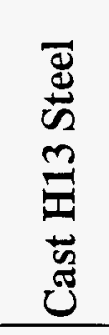 & 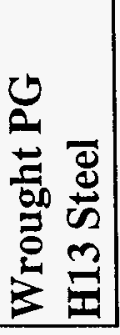 \\
\hline
\end{tabular}




\section{Table 2 - Structure and Hardness of Permanent Mold Materials Tested}

\begin{tabular}{|l|c|l|l|}
\hline Material & Hardness & \multicolumn{1}{|c|}{ Heat Treatment } & \multicolumn{1}{|c|}{ Microstructure } \\
\hline Alloy Gray Iron & $210 \mathrm{BHN}$ & Stress Relieved & $\begin{array}{l}\text { Flake graphite, pearlitic matrix } \\
\text { carbides }\end{array}$ \\
\hline $\begin{array}{l}\text { CWRU Alloy Gray } \\
\text { Iron-1 }\end{array}$ & $217 \mathrm{BHN}$ & As Cast & Flake graphite, pearlitic matrix \\
\hline $\begin{array}{l}\text { CWRU Alloy Gray } \\
\text { Iron-2 }\end{array}$ & $228 \mathrm{BHN}$ & As Cast & $\begin{array}{l}\text { Flake graphite, pearlitic bainitic } \\
\text { matrix }\end{array}$ \\
\hline $\begin{array}{l}\text { Compacted } \\
\text { Graphite Iron }\end{array}$ & $235 \mathrm{BHN}$ & Stress Relieved & $\begin{array}{l}\text { Compacted graphite, pearlitic } \\
\text { matrix }\end{array}$ \\
\hline Ductile Iron & $273 \mathrm{BHN}$ & Stress Relieved & $\begin{array}{l}\text { Spheroidal graphite, pearlitic } \\
\text { matrix }\end{array}$ \\
\hline CWRU Ductile Iron & $245 \mathrm{BHN}$ & $\begin{array}{l}\text { As Cast } \\
\text { Cast 4140 Steel }\end{array}$ & $\begin{array}{l}\text { Spheroidal graphite, pearlitic- } \\
\text { bainitic matrix }\end{array}$ \\
\hline Cast H13 Steel & $46 \mathrm{HRC}$ & $\begin{array}{l}1600^{\circ} \text { F Quenched and } \\
\text { Tempered }\end{array}$ & Tempered martensite \\
\hline $\begin{array}{l}\text { Wrought PG H13 } \\
\text { Steel }\end{array}$ & $47 \mathrm{HRC}$ & $\begin{array}{l}1900^{\circ} \text { F Oil Quenched } \\
\text { and Double Tempered }\end{array}$ & Tempered martensite \\
\hline
\end{tabular}




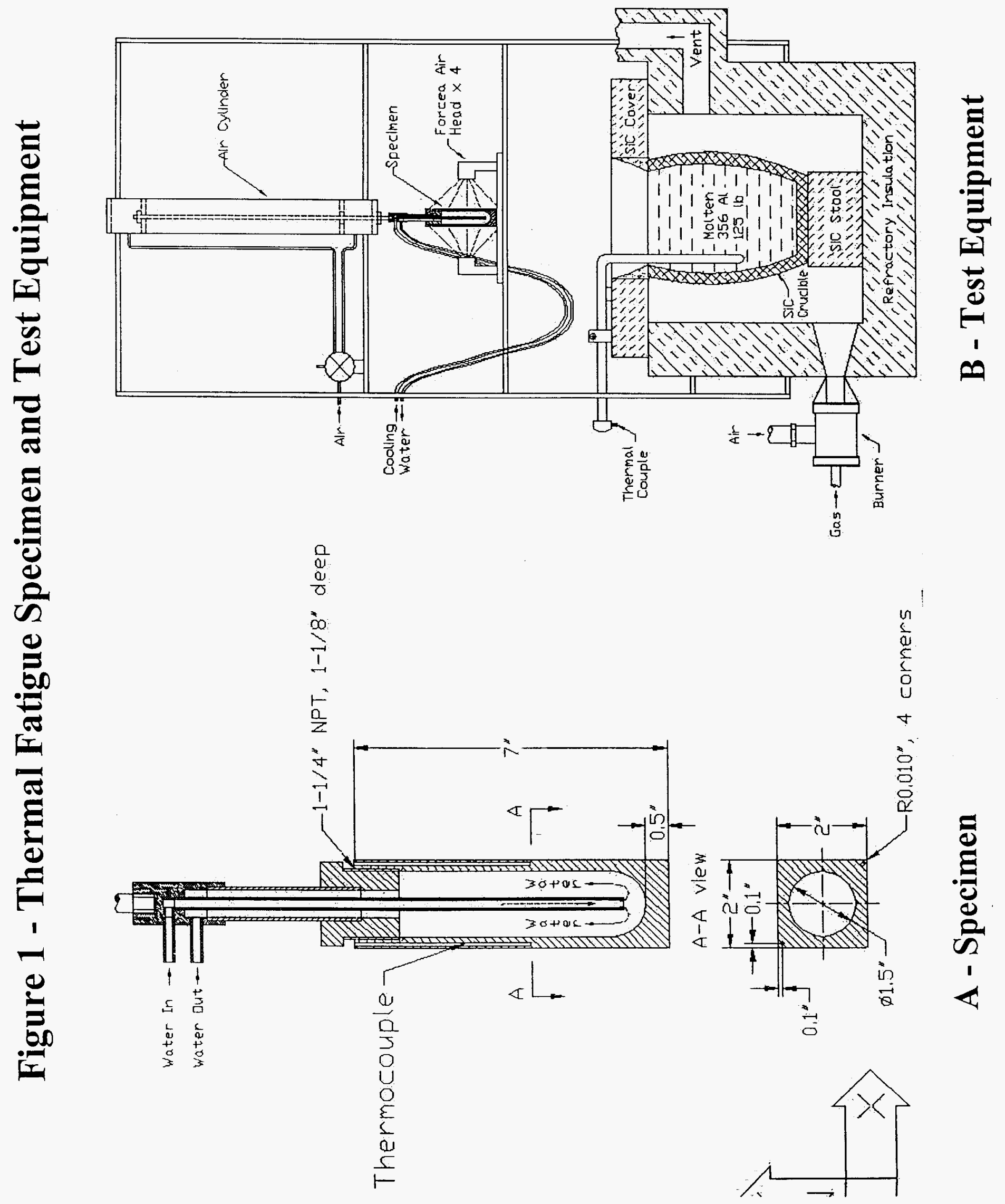




\section{Figure 2 - Thermal Fatigue Testing Cycles for Die and Permanent Molds Materials}

\section{$\underline{\text { For die casting dies }}$}

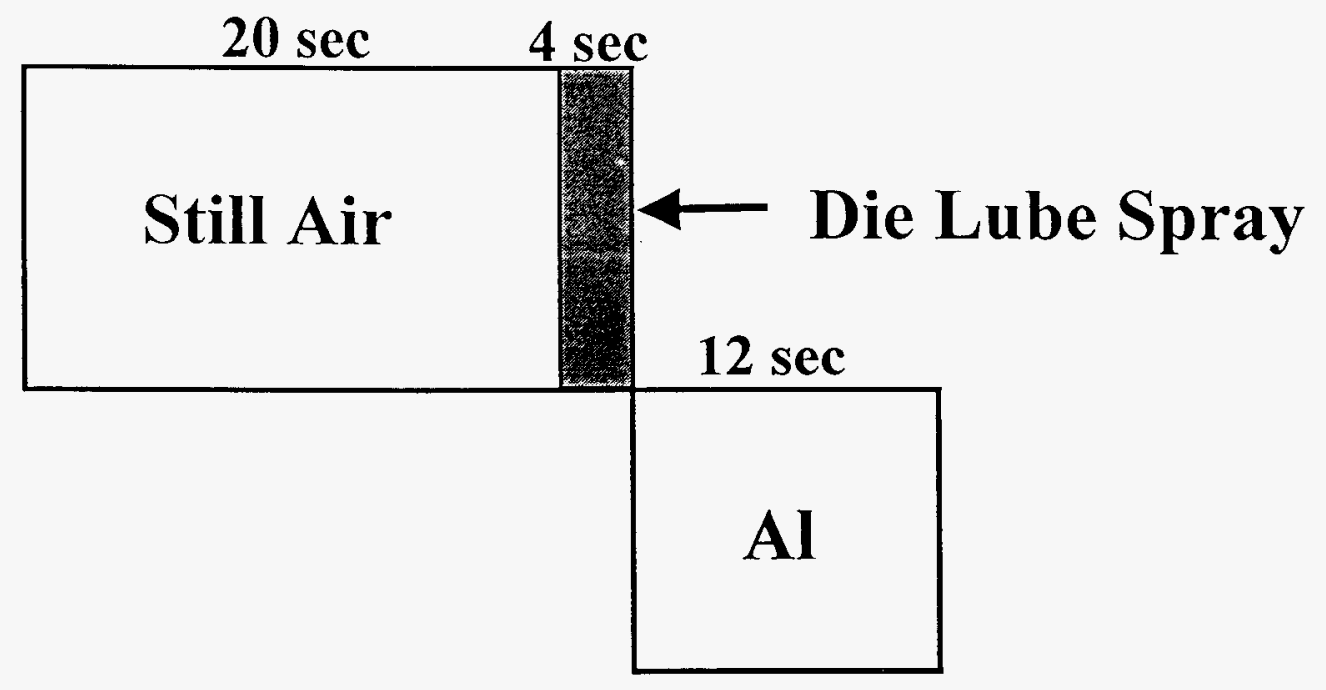

For permanent molds

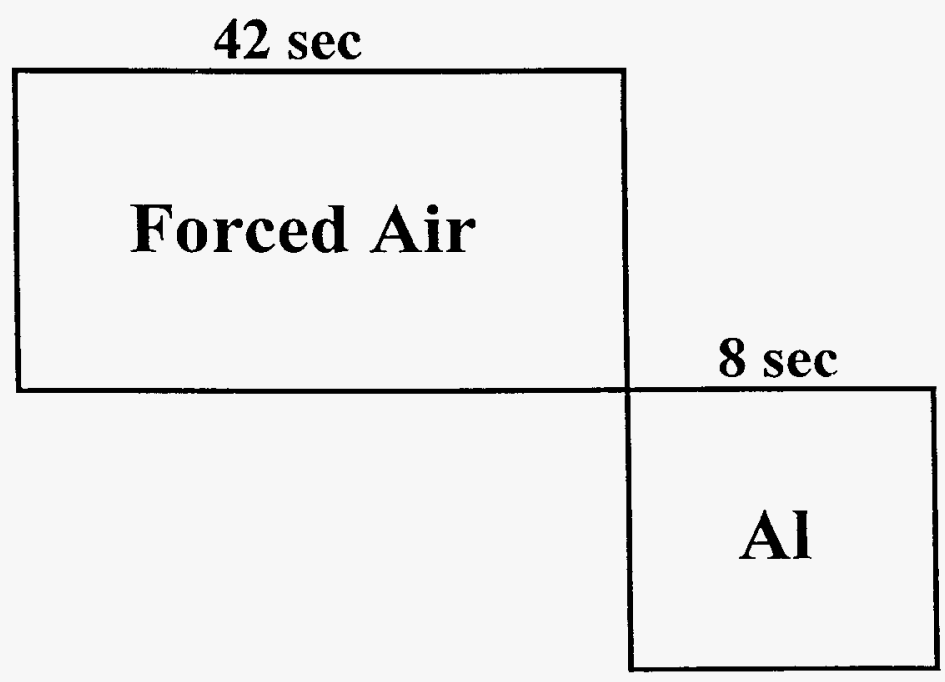

Note: Molten $\mathrm{Al}$ is at 1350 Fahrenheit Samples are cooled internally with water 
Figure 3 - Thermal Fatigue Cycles for Evaluation of Die Casting Dies and Permanent Mold Materials

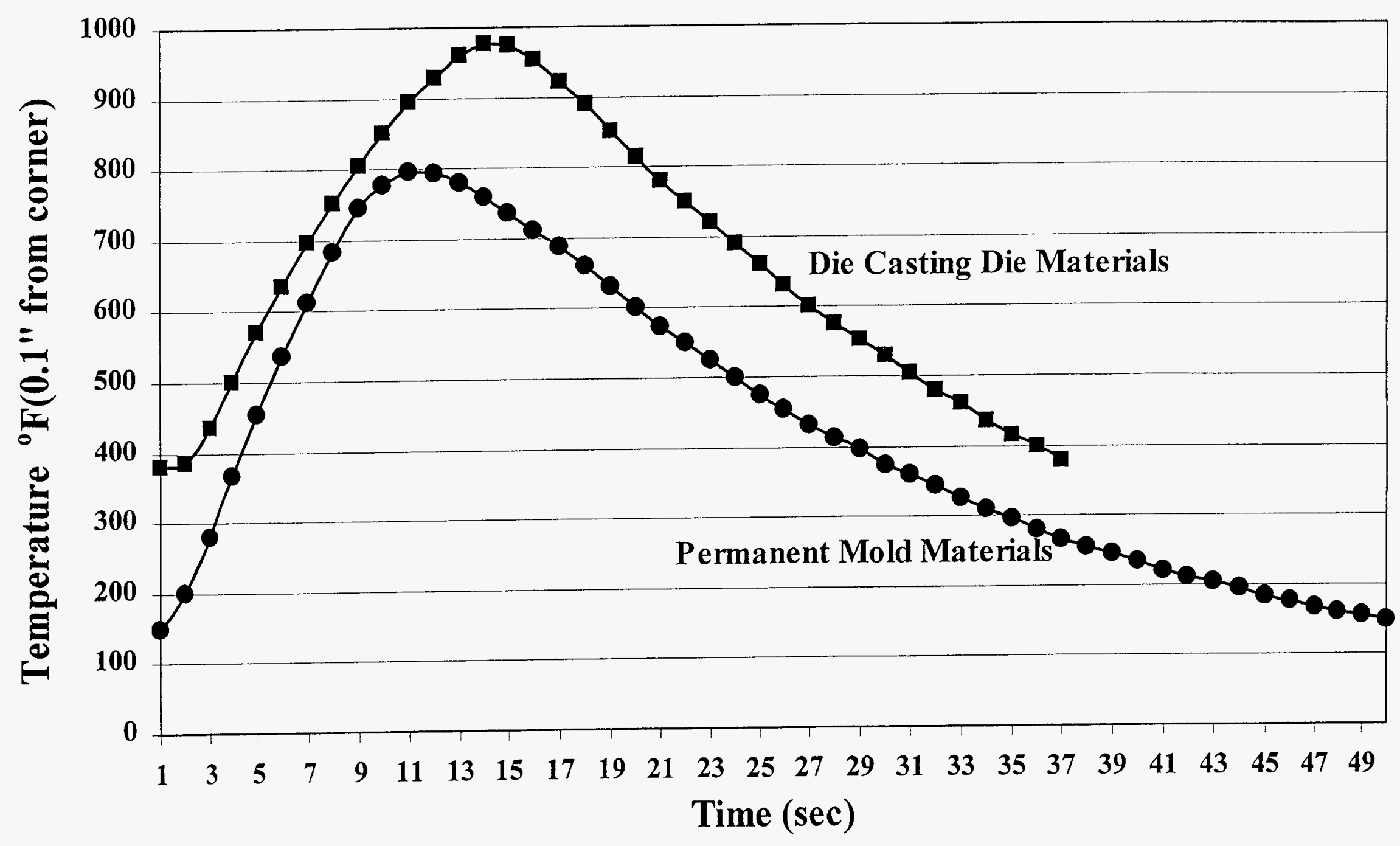


Figure 4 - Total Crack Area of Ductile Iron and Gray Iron

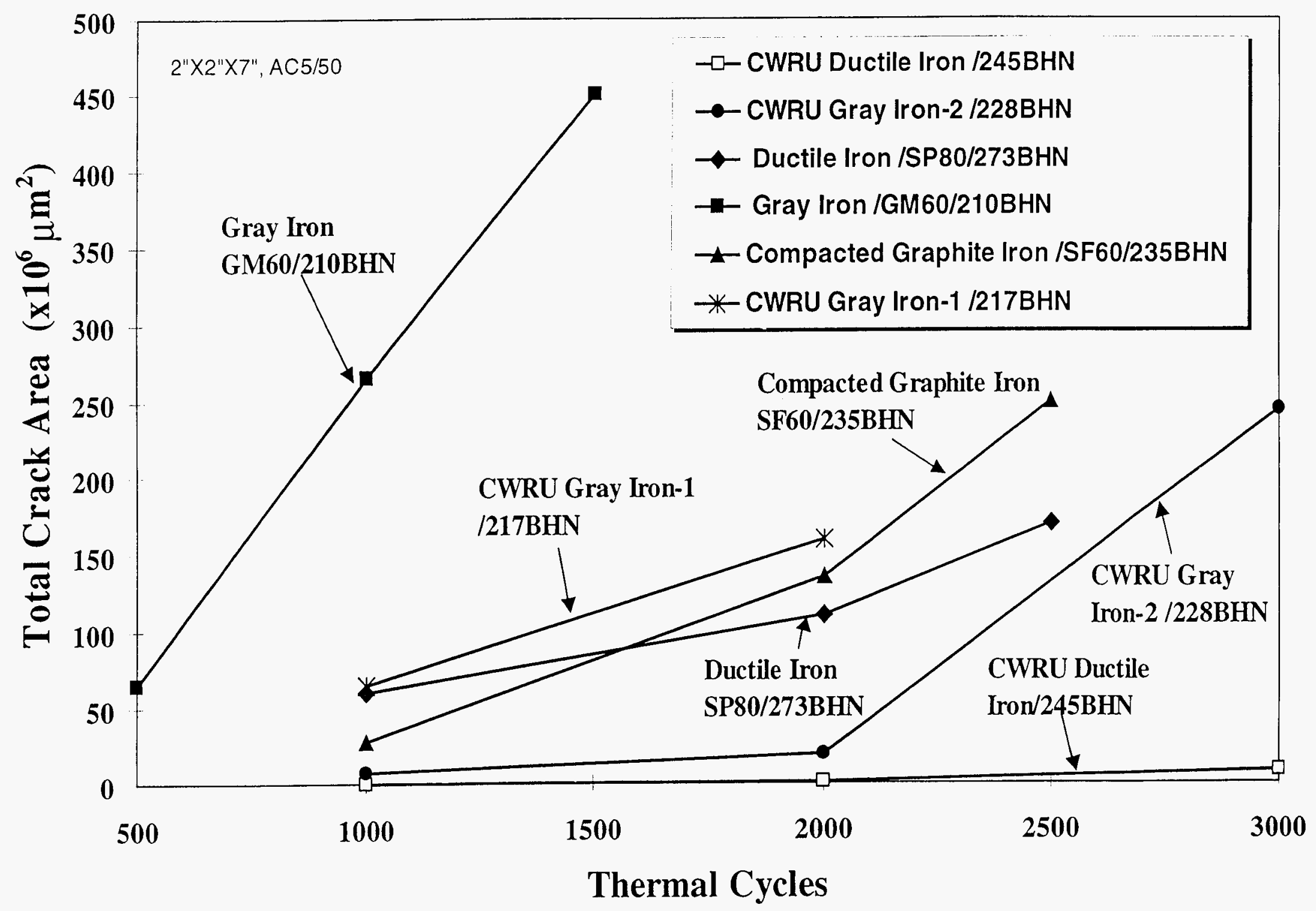


Figure 5 - Average Maximum Crack Length of Ductile Iron and Gray Iron

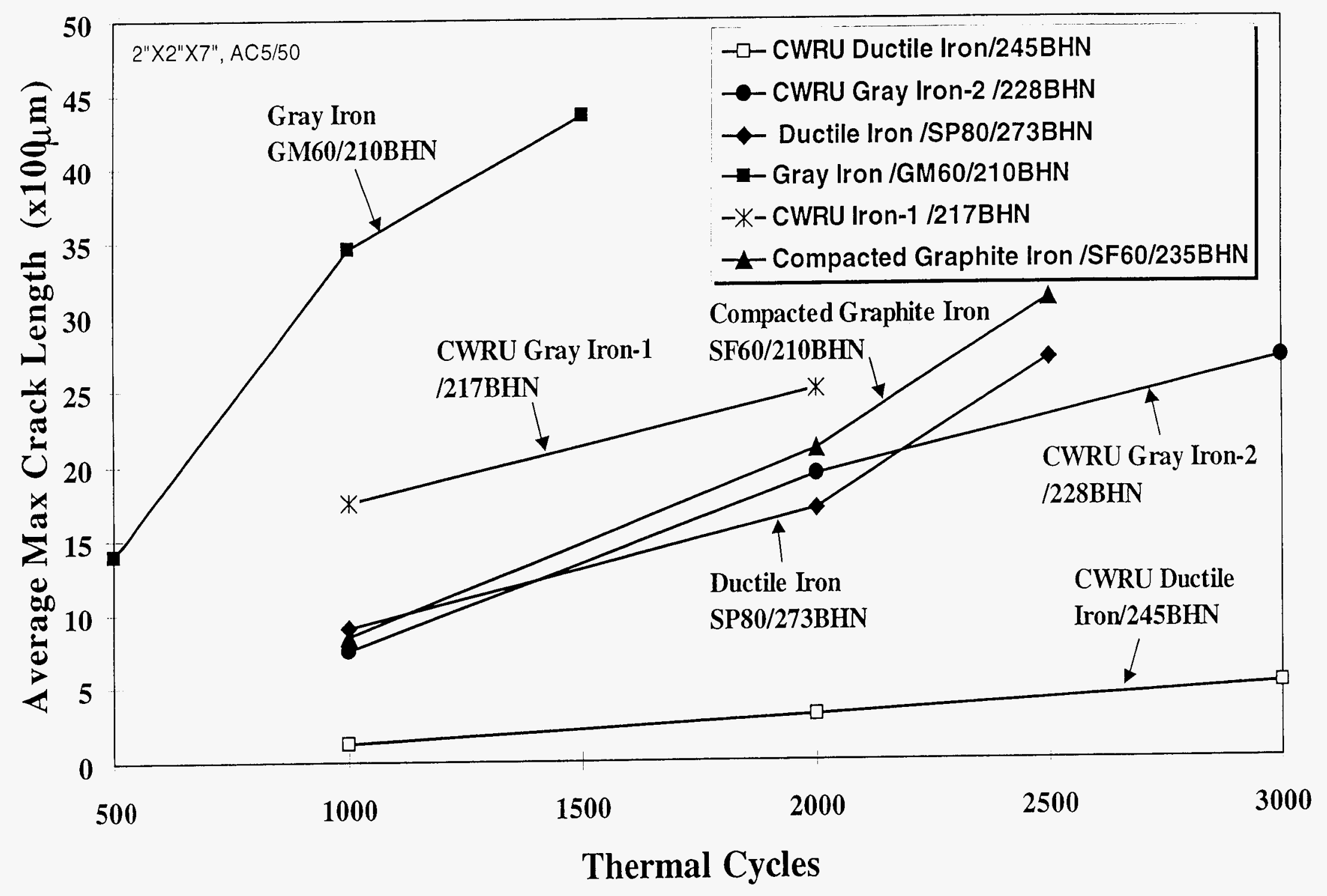




\section{Figure 6 - Total Crack Area of Permanent Mold Materials}

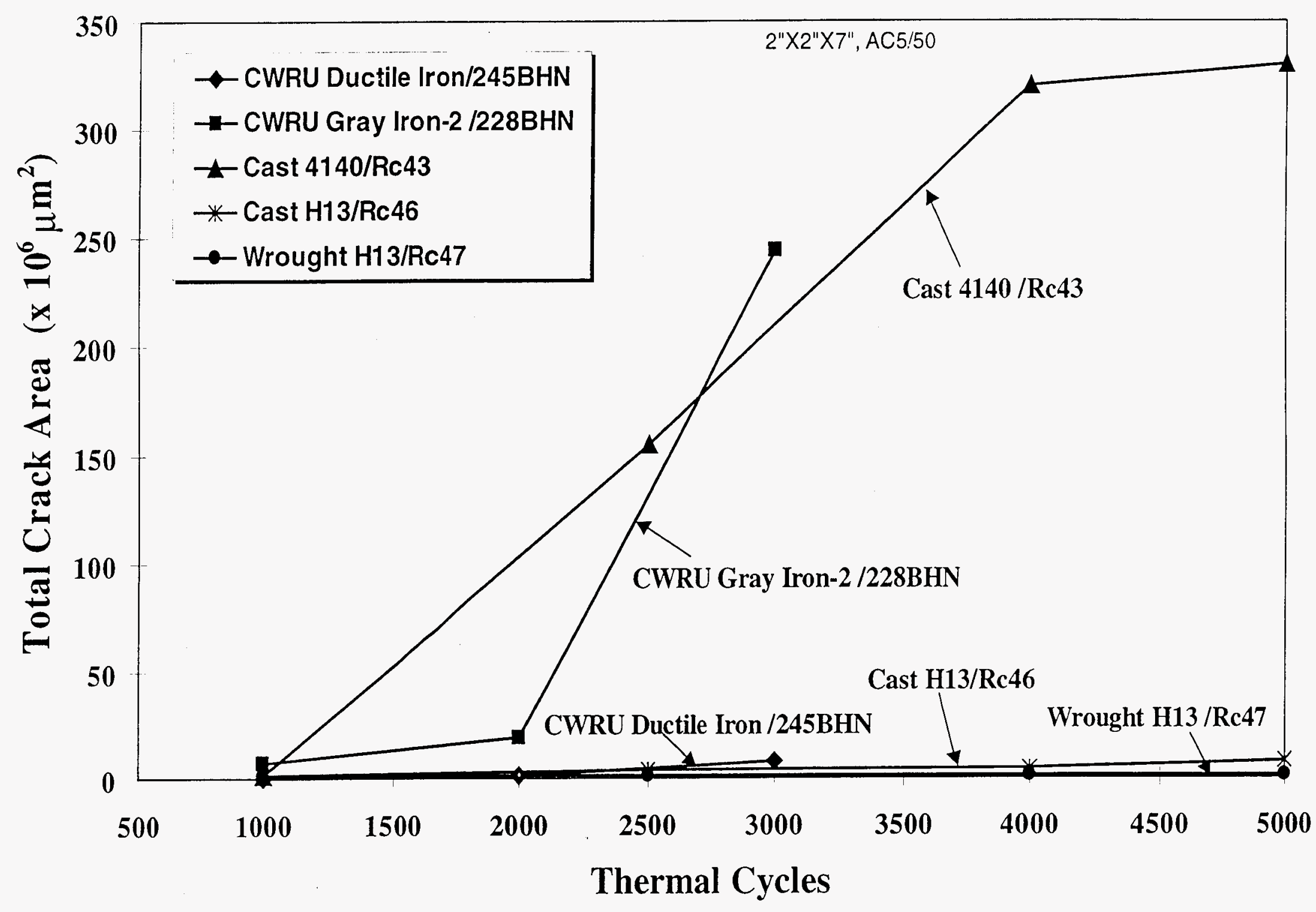




\section{Figure 8 - Thermal Fatigue Cracks in CWRU Gray Iron after 1000 Cycles}
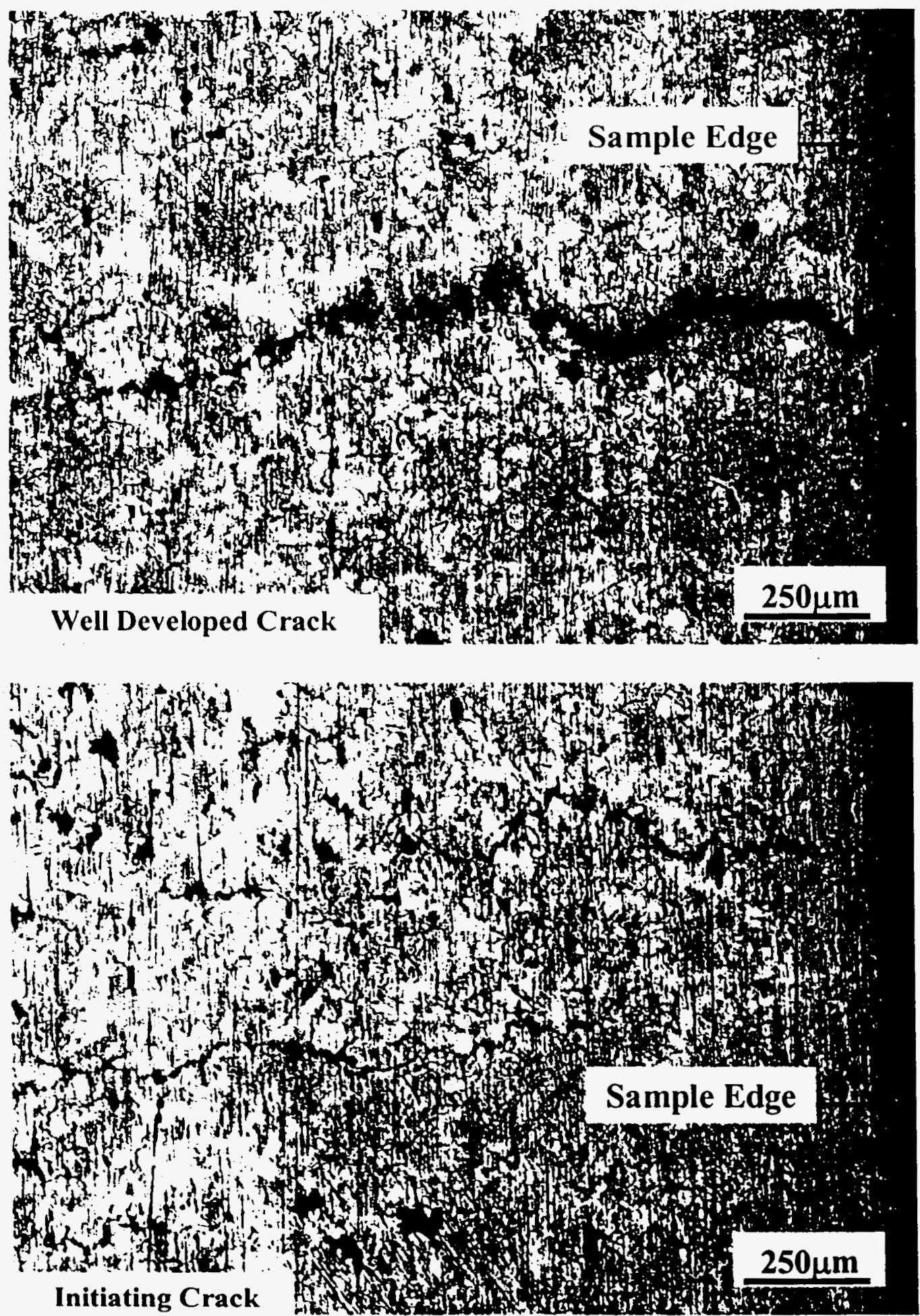
Figure 9 - Microstructure of CWRU Gray Iron for Thermal Fatigue Testing
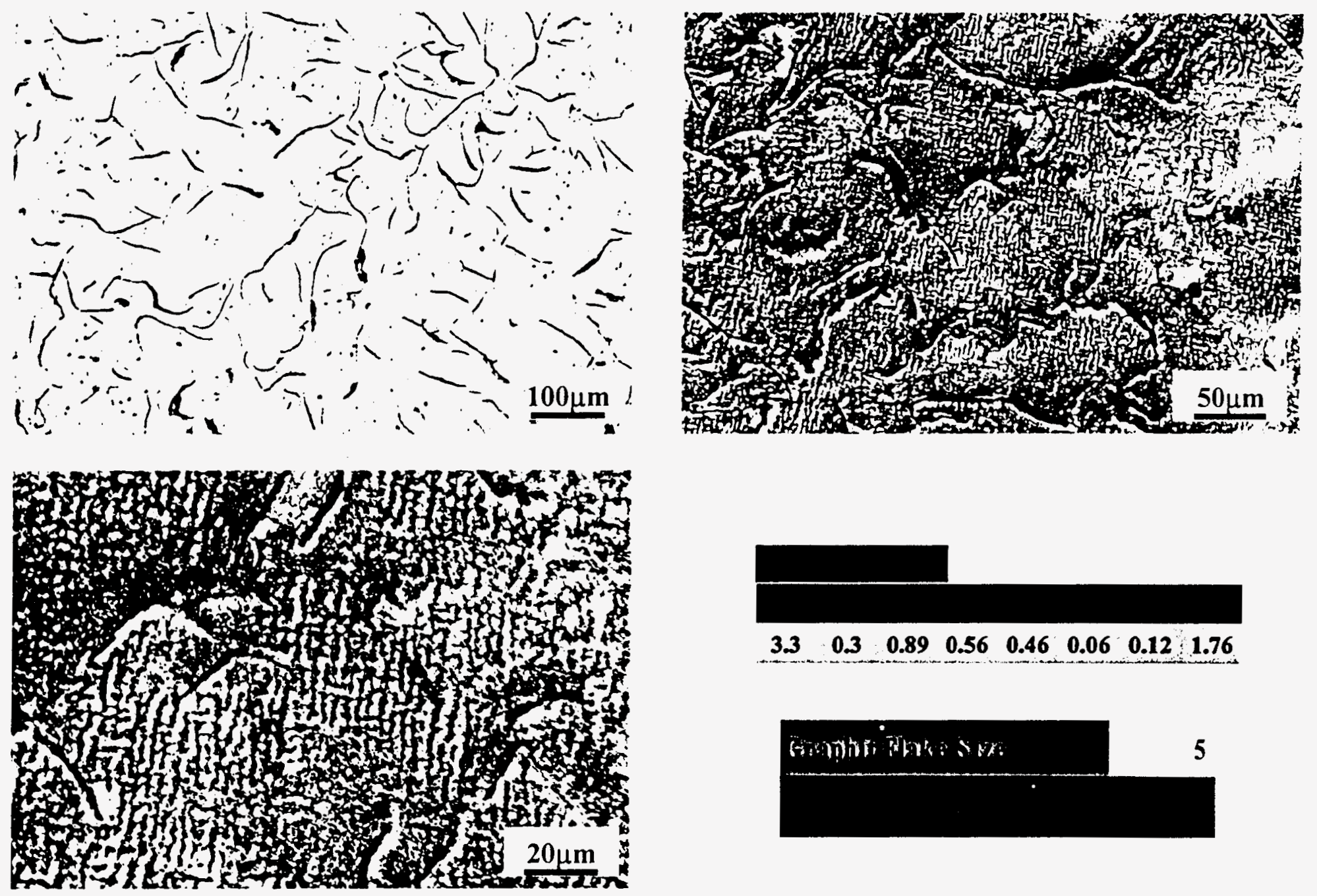


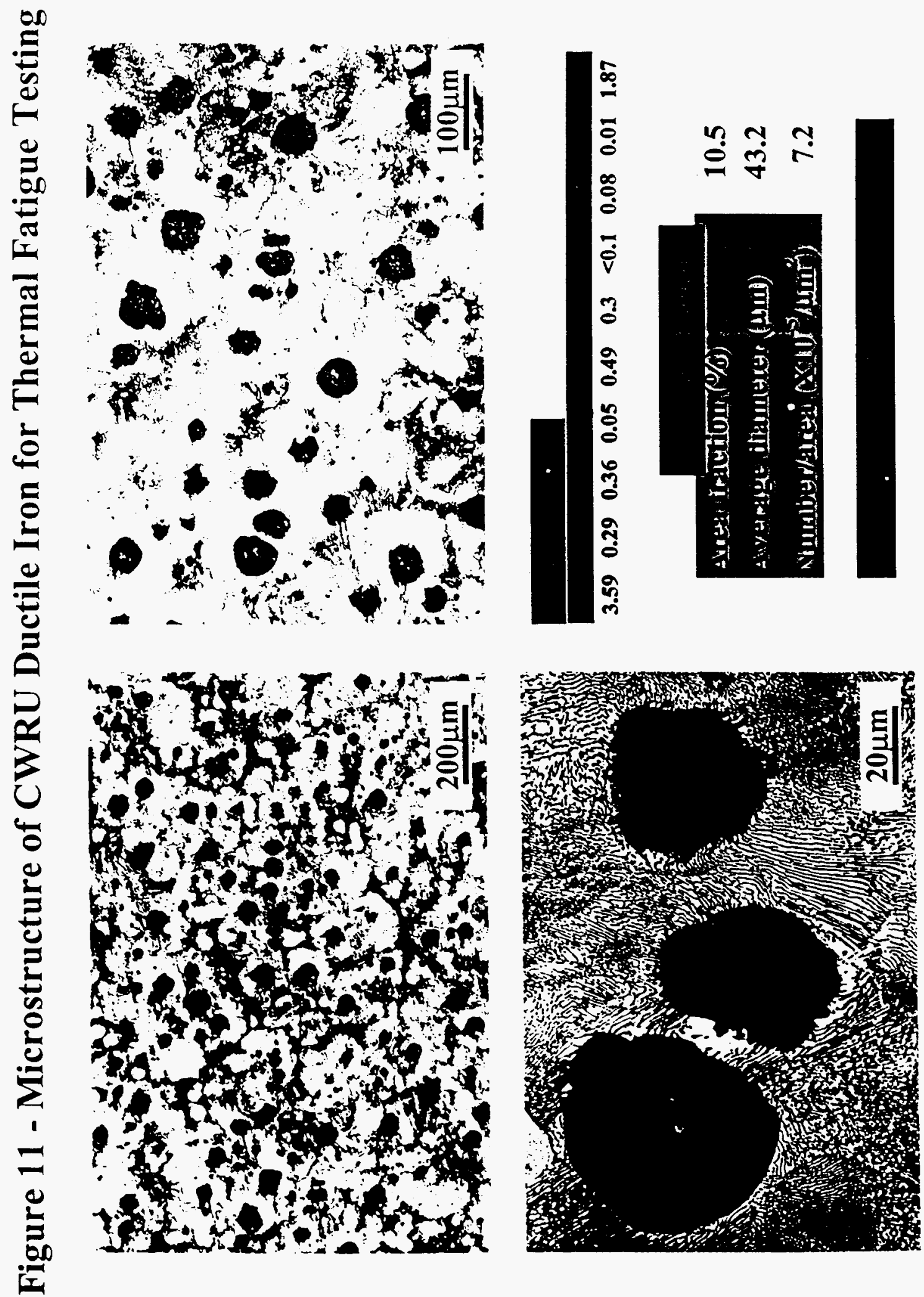




\section{Figure 12 - Segregation in CWRU Ductile Iron for Thermal Fatigue Testing}
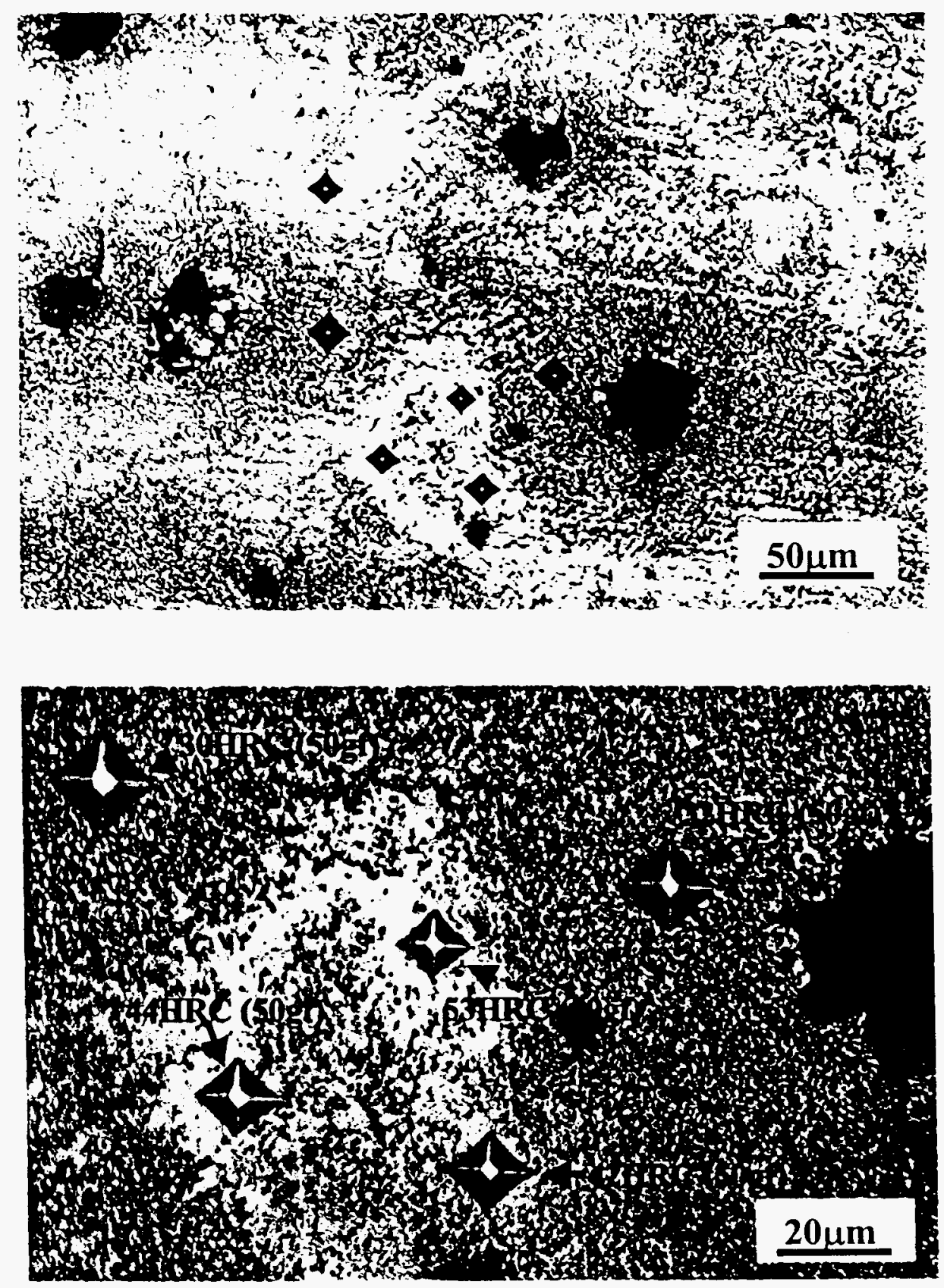
TABLES AND FIGURES FOR PART 2

EVALUATION OF COATINGS FOR PERMANENT MOLDS 
Table 1 - Typical Compositions of Coatings for Permanent Molds

\begin{tabular}{|c|c|c|c|c|c|c|c|c|c|c|}
\hline $\begin{array}{l}\text { Coating } \\
\text { No. }\end{array}$ & $\begin{array}{l}\text { Sodium } \\
\text { silicate }\end{array}$ & $\begin{array}{l}\text { White } \\
\text { Coating }\end{array}$ & Fire-clay & $\begin{array}{l}\text { Metal } \\
\text { oxide }\end{array}$ & \begin{tabular}{|c|}
$\begin{array}{c}\text { Diatomaceous } \\
\text { earth }\end{array}$ \\
\end{tabular} & $\begin{array}{l}\text { Soap - } \\
\text { stone(a) }\end{array}$ & Talc (a) & Mica (a) & Graphite & $\begin{array}{l}\text { Boric } \\
\text { acid }\end{array}$ \\
\hline 1 & 2 & & 4 & & & & & & 1 & \\
\hline 2 & & & 4 & & & & & & & \\
\hline 3 & 8 & 7 & & & & & & & & 7 \\
\hline $4(b)$ & 12 & 9 & & & & & & & & \\
\hline 5 & 5 & 11 & & 2 & & & 5 & & & \\
\hline 6 & 9 & & 4 & & & 14 & & & & \\
\hline 7 & 11 & & & 17 & & & & & & \\
\hline 8 & & & 4 & & & 23 & & 5 & & \\
\hline 9 & 7 & & $\cdot \quad 1$ & & 5 & 23 & & 2 & & \\
\hline 10 & 23 & & & & 41 & & 20 & & & \\
\hline 11 & 30 & & & & & & 10 & & & \\
\hline 12 & 18 & & & & & & & & & \\
\hline 13 & 8 & & & 60 & & & & & & \\
\hline 14 & 7 & & & & & & 62 & & & \\
\hline 15 & 20 & 53 & & & & & & & & \\
\hline
\end{tabular}

(a) Serve also as an insulator (b) Plus silicon carbide, $2 \%$ by weight, for wear resistance Composition, \% by weight (remainder, water) 
Table 2 - Properties of Typical Refractory Fillers

\begin{tabular}{|c|c|c|c|}
\hline Refractory & Chemical formula & Density $(\mathrm{g} / \mathrm{cC})$ & Melting point $\left({ }^{\circ} \mathrm{C}\right)$ \\
\hline Coke & $\mathrm{C}$ & $1.6-1.8$ & $\mathrm{~N} / \mathrm{A}$ \\
\hline Graphite & $\mathrm{C}$ & $2.1-2.3$ & 3000 \\
\hline Silica & $\mathrm{SiO}_{2}$ & 2.65 & 1800 \\
\hline Zircon & $\mathrm{ZrSiO}_{4}$ & $4.0-4.8$ & 2200 \\
\hline Mica & $\mathrm{KAl}_{2}\left(\mathrm{OH}_{1} \mathrm{~F}_{2} \mathrm{Si}_{3} \mathrm{AlO}_{10}\right.$ & 2.3 & $750-1100$ \\
\hline Calcined Magnesite & $\mathrm{MgO}$ & $3.5-3.7$ & 2800 \\
\hline Alumina silicate & $\mathrm{Al}_{2} \mathrm{O}_{3} \mathrm{SiO}_{2}$ & $2.6-3.0$ & $\mathrm{~N} / \mathrm{A}$ \\
\hline Chamotte & $\mathrm{N} / \mathrm{A}$ & 2.6 & 1700 \\
\hline Alumina & $\mathrm{Al}_{2} \mathrm{O}_{3}$ & 3.9 & 2050 \\
\hline
\end{tabular}


Table 3 - Suitability of Fillers for Common Casting Alloys

\begin{tabular}{|c|c|c|c|c|c|c|c|c|c|}
\hline Alloy & Coke & Graphite & Silica & Zircon & Mica & Talc & Calcined Alumina & Magnesite & Chamotte \\
\hline Cast Steel & 0 & 0 & 0 & + & - & - & 0 & - \\
\hline Alloy Cast Steel & 0 & 0 & 0 & - & - & - & + & + \\
\hline Cast Iron & - & + & - & + & 0 & + & - & 0 & - \\
\hline Nodular Iron & - & + & + & + & 0 & + & - & 0 & - \\
\hline Malleable Iron & + & + & + & + & 0 & + & - & 0 & - \\
\hline Copper Alloy & + & + & - & 0 & + & + & 0 & + \\
\hline Al Alloy & - & + & - & + & + & + & - & 0 \\
\hline Mg Alloy & 0 & 0 & - & - & + & + & + & - \\
\hline
\end{tabular}

+ Applicable

O Possible under certain conditions

- Unsuitable or uncommon 
Table 4. Chemical Composition of H-13 Steel

\begin{tabular}{|c|c|}
\hline Element & Composition in Weight Percent \\
\hline $\mathrm{C}$ & 0.39 \\
\hline $\mathrm{Mn}$ & 035 \\
\hline $\mathrm{Si}$ & 1.00 \\
\hline $\mathrm{Cr}$ & 5.25 \\
\hline $\mathrm{Mo}$ & 1.45 \\
\hline $\mathrm{V}$ & 1.00 \\
\hline $\mathrm{S}$ & $0.005 \max$. \\
\hline $\mathrm{P}$ & $0.025 \max$. \\
\hline
\end{tabular}


Table 5 - Physical Properties and Characteristics of Coatings

\begin{tabular}{|c|c|c|c|c|c|}
\hline Coating & Composition & Insulation & Durability & Texture & Application \\
\hline DYCOTE 11 & Graphite & Very Low & Low & Smooth & Release \\
\hline DYCOTE 8 & N/A & Very High & Very High & Medium/Rough & Risers \\
& Gates / Primer \\
\hline DYCOTE & $\begin{array}{c}\text { Talc } \\
\text { Mica } \\
\text { Magnesium } \\
\text { Oxide }\end{array}$ & High & Medium & Rough & Aids Metal Flow \\
& $\begin{array}{c}\text { Zircon } \\
\text { Iron Oxide }\end{array}$ & N/A & Very High & Smooth & Base Coat \\
\hline DYCOTE R87 & & & & Top Coat \\
\hline
\end{tabular}




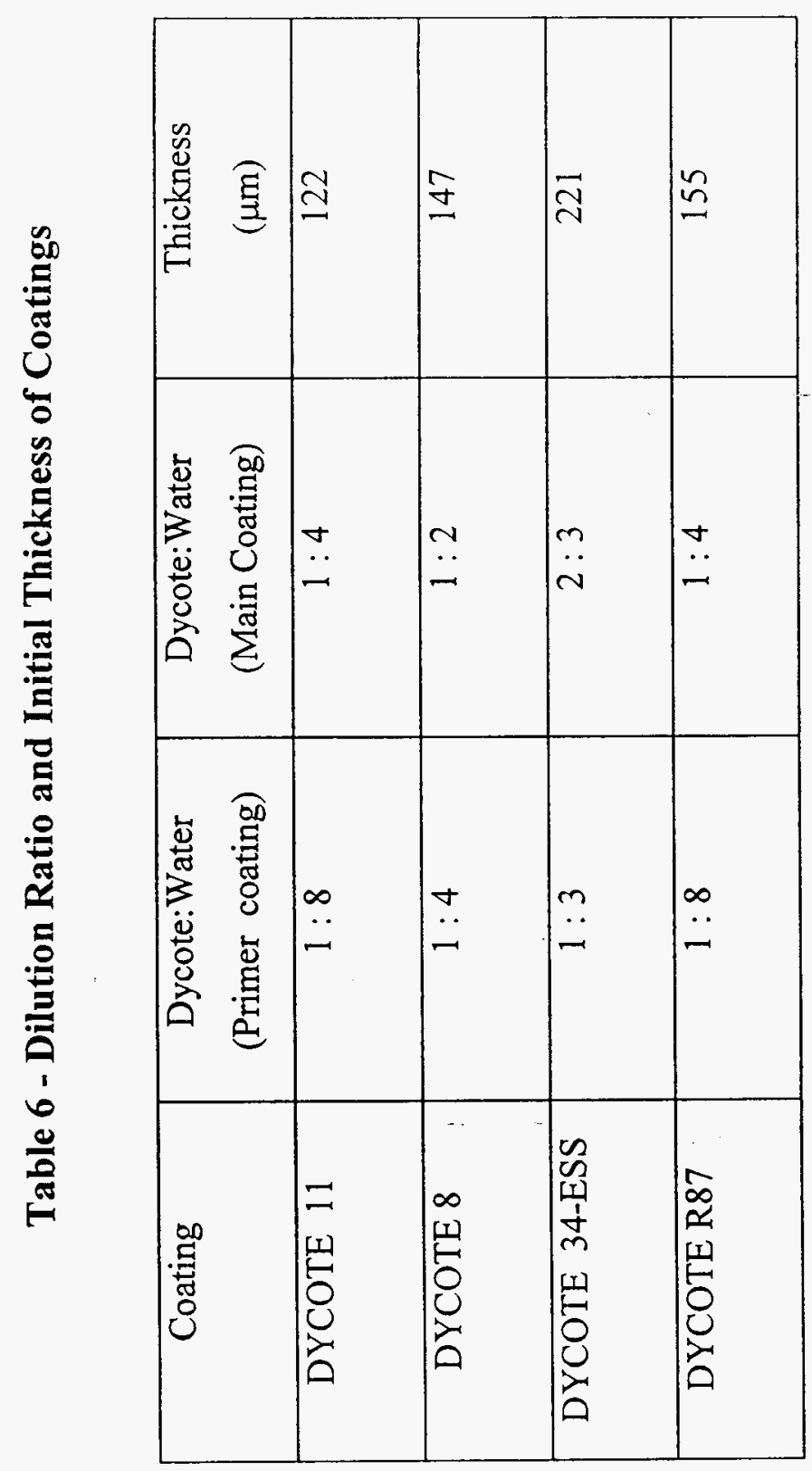


Table 7 - Temperature Difference of Uncoated Corner During Immersion

\begin{tabular}{|c|c|c|c|c|c|}
\hline \multirow[t]{2}{*}{ Coating } & \multirow[t]{2}{*}{ Cycles } & \multicolumn{3}{|c|}{ Average Temperature of Uncoated Corner $\left({ }^{\circ} \mathrm{F}\right)$} & \multirow{2}{*}{$\begin{array}{c}\text { Molten Al Temperature } \\
\left({ }^{\circ} \mathrm{F}\right)\end{array}$} \\
\hline & & Maximum & Minimum & Difference & \\
\hline \multirow{4}{*}{ Dycote 8} & $11-20$ & 829 & 251 & 578 & 1357 \\
\hline & $51-60$ & 813 & 255 & 558 & 1350 \\
\hline & $91-100$ & 803 & 254 & 549 & 1345 \\
\hline & average & 815 & 253 & 562 & 1351 \\
\hline \multirow{4}{*}{$\begin{array}{l}\text { Dycote } \\
\text { 34-ESS }\end{array}$} & $11-20$ & 808 & 258 & 550 & 1353 \\
\hline & $51-60$ & 799 & 259 & 540 & 1347 \\
\hline & $91-100$ & 783 & 256 & 527 & 1330 \\
\hline & average & 797 & 250 & 566 & 1346 \\
\hline \multirow{4}{*}{$\begin{array}{l}\text { Dycote } \\
\text { R87 }\end{array}$} & $11-20$ & 854 & 253 & 601 & 1357 \\
\hline & $51-60$ & 768 & 249 & 519 & 1351 \\
\hline & $91-100$ & 767 & 251 & 516 & 1347 \\
\hline & average & 796 & 251 & 545 & 1352 \\
\hline \multirow{4}{*}{ Dycote 11} & $11-20$ & 791 & 234 & 557 & 1369 \\
\hline & $51-60$ & 743 & 235 & 508 & 1339 \\
\hline & $91-100$ & 758 & 233 & 525 & 1344 \\
\hline & average & 764 & 234 & 530 & 1351 \\
\hline
\end{tabular}


Table 8 - Coating Characteristic \&Temperature Difference of Coated Corner During Immersion

\begin{tabular}{|c|c|c|c|c|c|c|c|c|}
\hline \multirow[t]{2}{*}{ Coating } & \multirow[t]{2}{*}{ Cycles } & \multicolumn{3}{|c|}{ Average Temperature of Coated Corner $\left({ }^{\circ} \mathrm{F}\right)$} & \multirow{2}{*}{$\begin{array}{c}\text { Roughness } \\
\text { um }\end{array}$} & \multicolumn{2}{|c|}{ Coating Thickness (um) } & \multirow[t]{2}{*}{ Adhesion } \\
\hline & & Maximum & Minimum & Difference & & Initial & Final & \\
\hline \multirow{4}{*}{ Dycote 8} & $11-20$ & 298 & 158 & 140 & \multirow{4}{*}{50} & \multirow{4}{*}{147.3} & \multirow{4}{*}{58} & \multirow{4}{*}{ good } \\
\hline & $51-60$ & 371 & 168 & 203 & & & & \\
\hline & $91-100$ & 369 & 175 & 194 & & & & \\
\hline & average & 346 & 167 & 179.0 & & & & \\
\hline \multirow{4}{*}{$\begin{array}{l}\text { Dycote } \\
\text { 34-ESS }\end{array}$} & $11-20$ & 361 & 175 & 186 & \multirow{4}{*}{63} & \multirow{4}{*}{221} & \multirow{4}{*}{84} & \multirow{4}{*}{ good } \\
\hline & $51-60$ & 448 & 187 & 261 & & & & \\
\hline & $91-100$ & 461 & 190 & 271 & & & & \\
\hline & average & 399 & 170 & 229 & & & & \\
\hline \multirow{4}{*}{ Dycote R87 } & $11-20$ & 430 & 154 & 276 & \multirow{4}{*}{20} & \multirow{4}{*}{155} & \multirow{4}{*}{76} & \multirow{4}{*}{ good } \\
\hline & $51-60$ & 536 & 172 & 364 & & & & \\
\hline & $91-100$ & 578 & 180 & 398 & & & & \\
\hline & average & 515 & 169 & 346 & & & & \\
\hline \multirow{4}{*}{ Dycote 11} & $11-20$ & 539 & 187 & 352 & \multirow{4}{*}{31} & \multirow{4}{*}{122} & \multirow{4}{*}{30} & \multirow{4}{*}{ good } \\
\hline & $51-60$ & 545 & 190 & 355 & & & & \\
\hline & $91-100$ & 569 & 179 & 390 & & & & \\
\hline & average & 551 & 185 & 366 & & & & \\
\hline
\end{tabular}



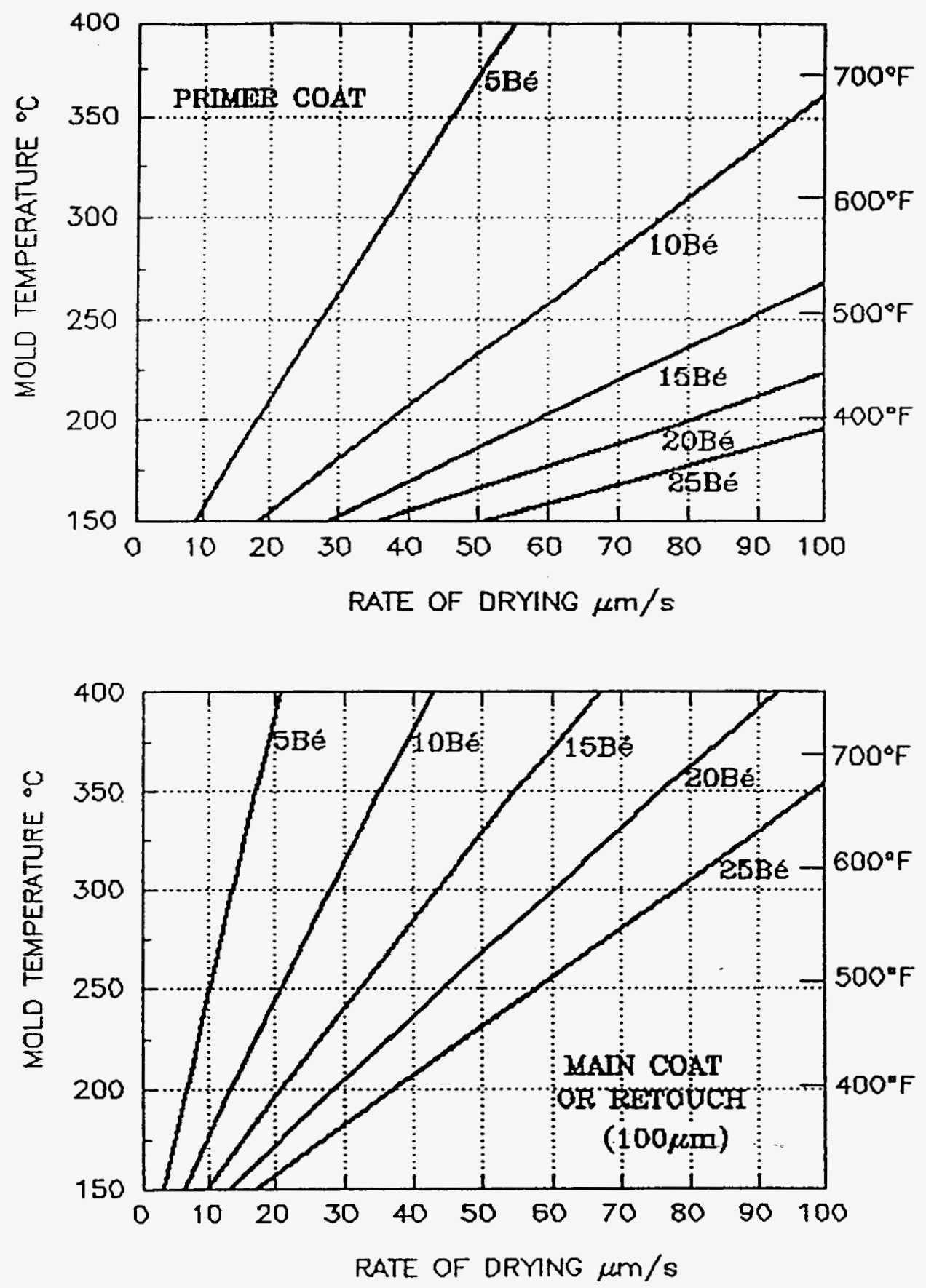

Figure 1 - Relationships between slurry density, mold temperature and rate of drying for the initial passes (top) and after a $100 \mu \mathrm{m}$ build-up (bottom) - Talc based coating $(d=2.7)$ 
Eliptical halo $39 \times 13 \mathrm{cn}=306.15 \mathrm{~cm}$ - Spray pun flow rutec $60 \mathrm{~cm} 3 \mathrm{~min}$ Slurry solld $\%$ by colunc= $.07 \%$ ( 15.4 be for $d=2.7$ ) - 260 in droplets

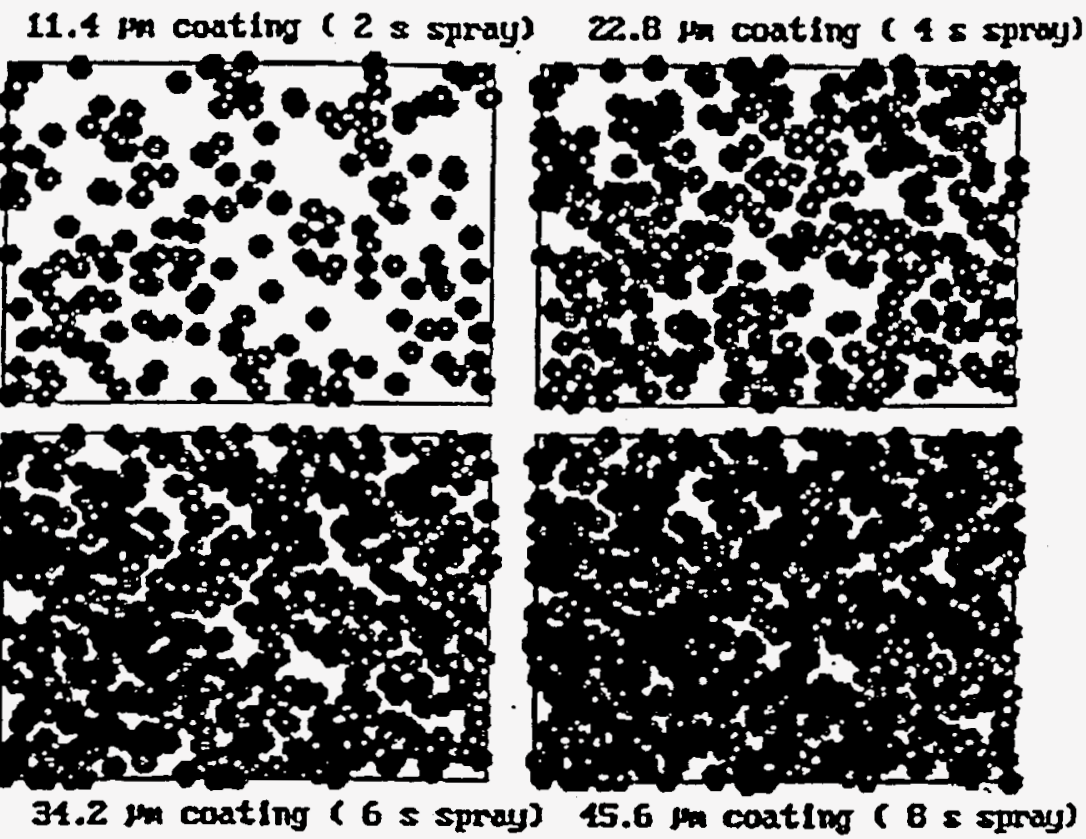

Ell Iptical halo $39 \times 13 \mathrm{~cm}=306.15 \mathrm{~cm}$ - Spray gun flou rate $=69 \mathrm{ck} 3 \mathrm{~min}$ Slurry solid \% by coluac= .67 \% ( 15.4 be for $d=2.7)-100$ on droplets

$11.4 \mathrm{~m}$ coating ( 2 s spray) $22.8 \mathrm{~m}$ coating ( $1 \times$ spray)
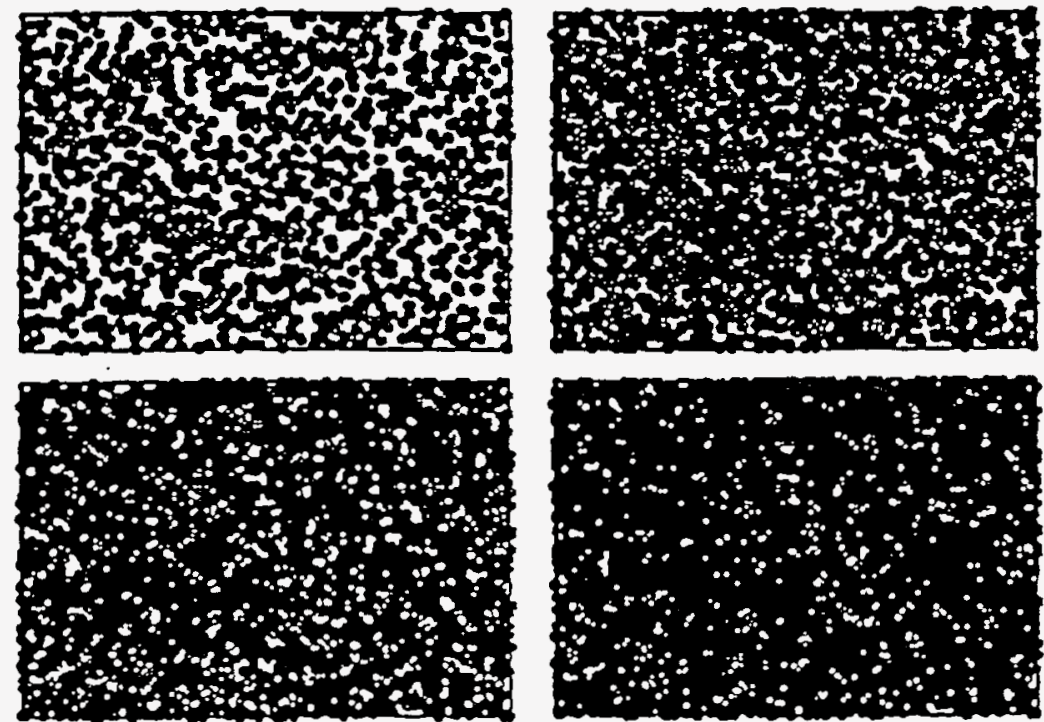

$34.2 \mathrm{~m}$ costing ( 6 s sproy) $45.6 \mathrm{~mm}$ conting ( 8 s spray)

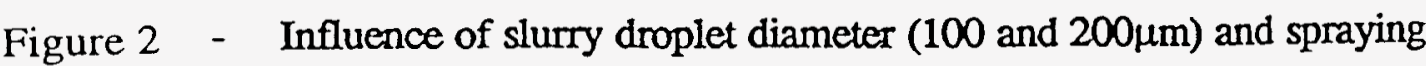
parameters on mold covering. 


\section{ROTATION OF SPRAY GUN}
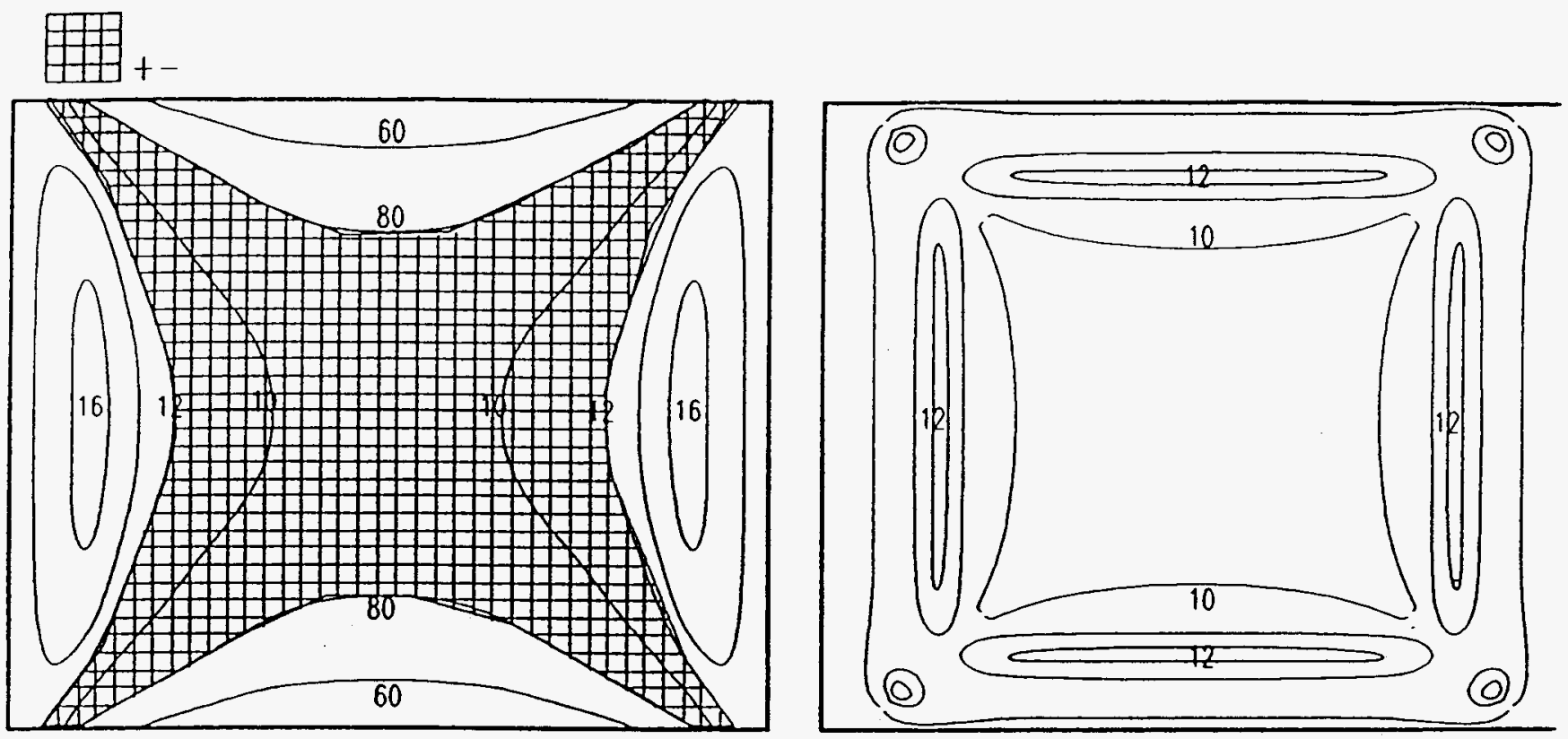

HORIZONTAL PASSES

CROSSED

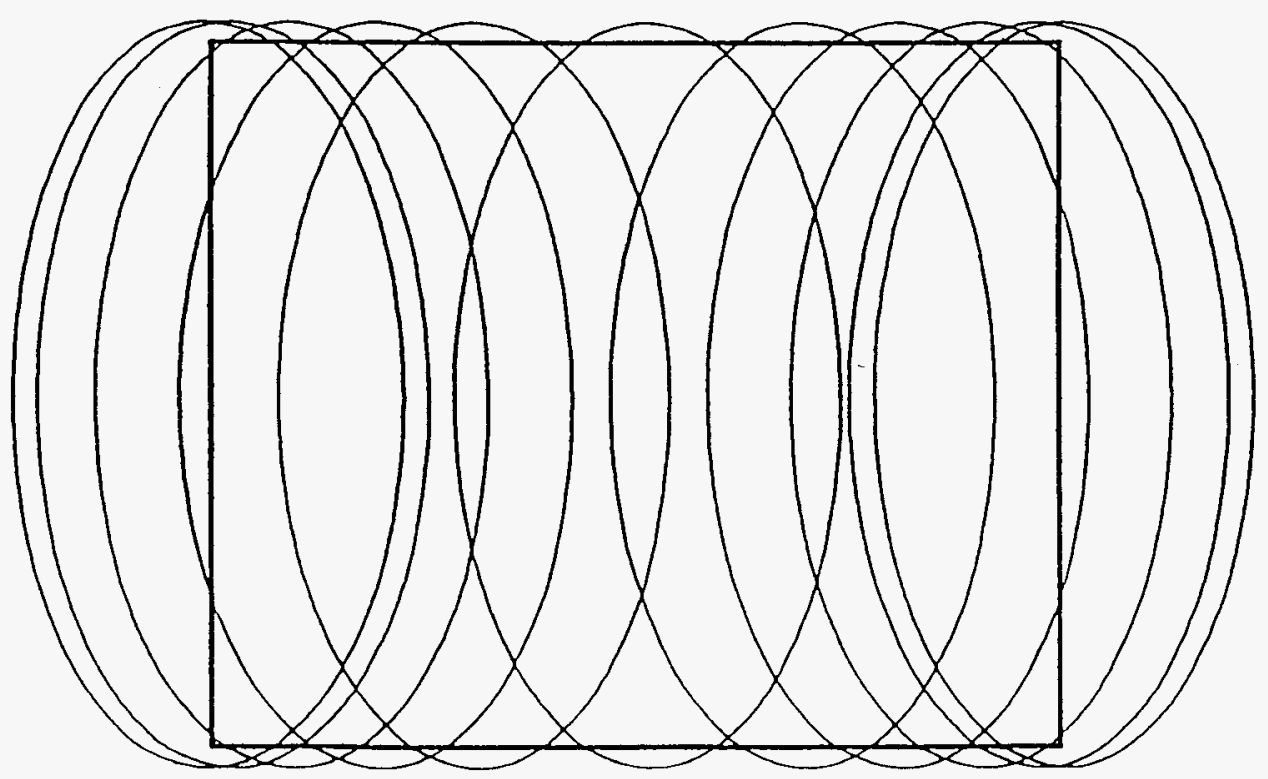

Figure 3 - Distribution of the coating deposit when the spray nozzle is rotated about a vertical axis, in \% of average thickness. Note more even distribution when crossing passes. 
TRANSLATION OF SPRAY GUN

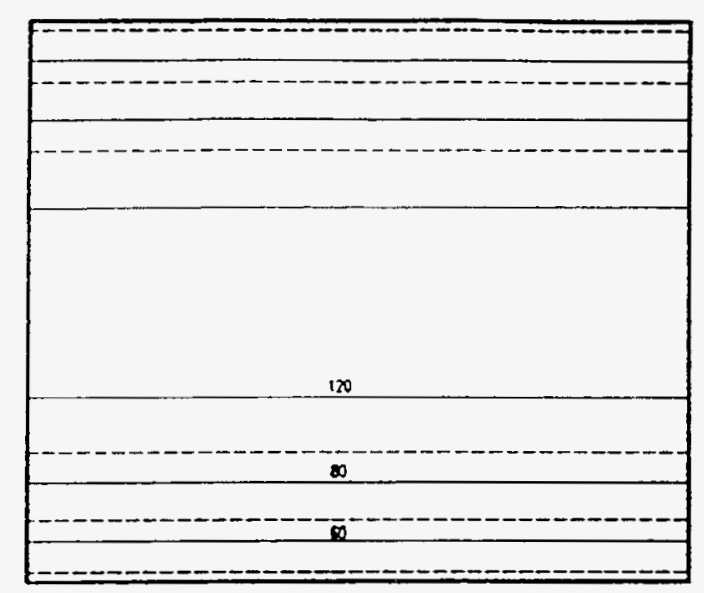

HORZONTAL PASSES

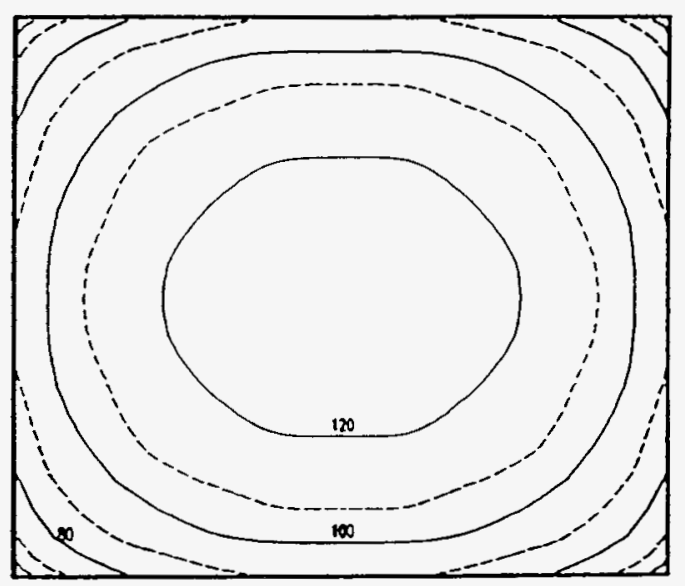

CROSSED PASSES

CONSTANT SPRAY GUN VELOCTY

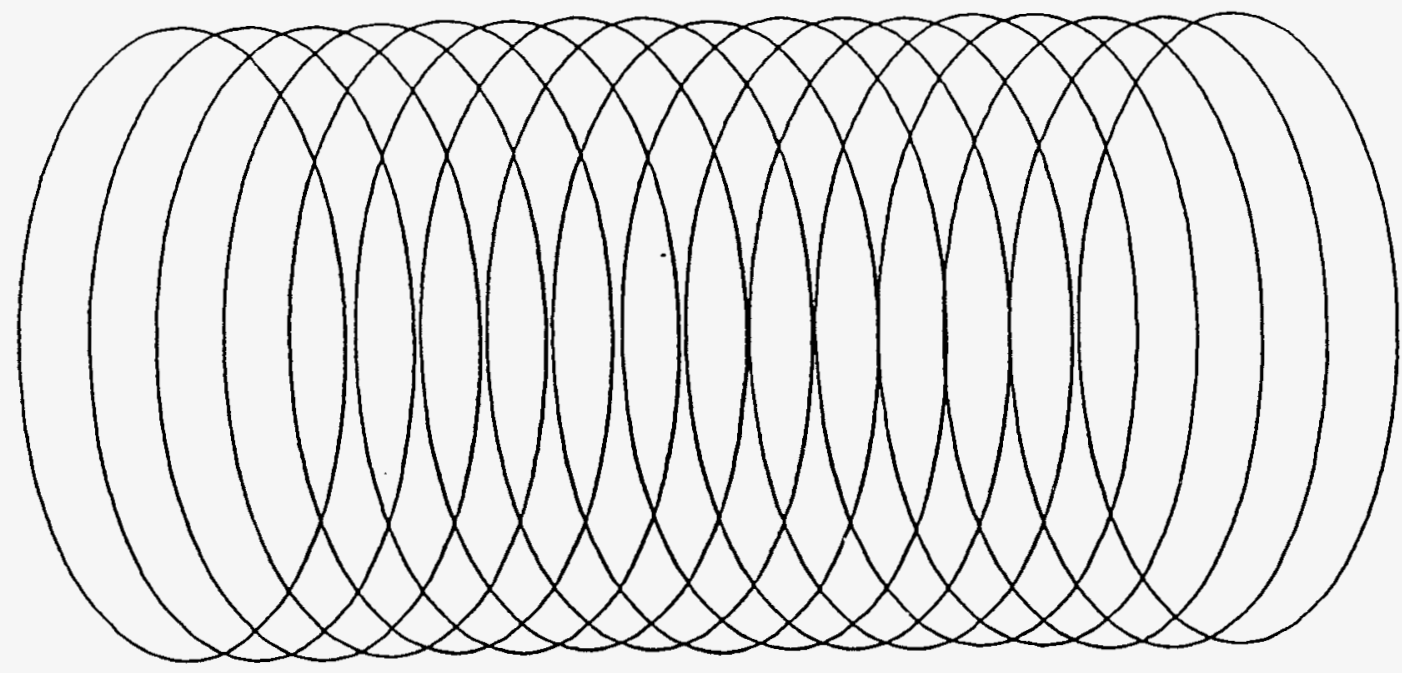

Figure 4 - Distribution of the coating deposit when the spray nozzle is moved at a constant velocity . Influence of crossing passes. 


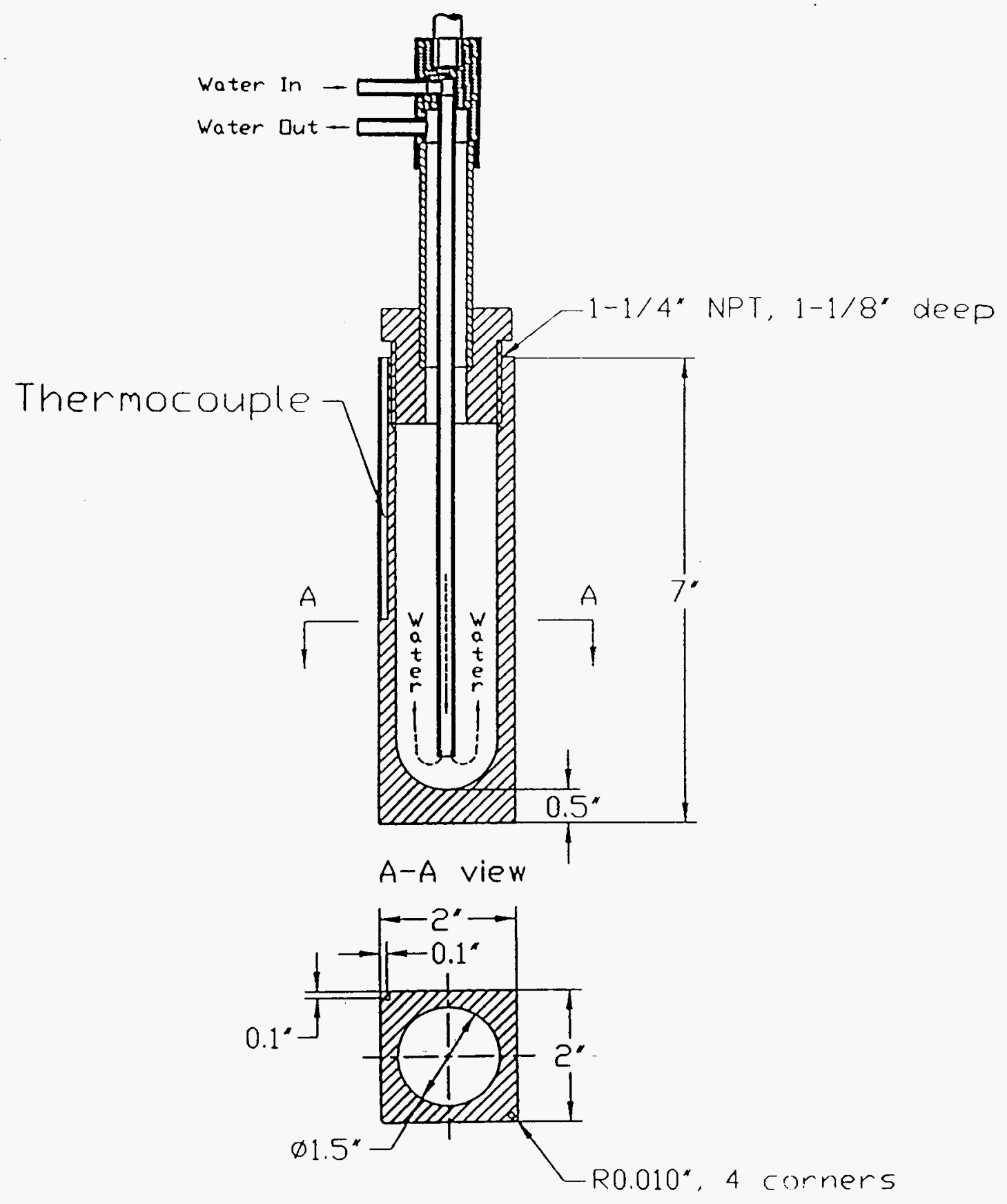

Figure-5 Configuration of the specimen 


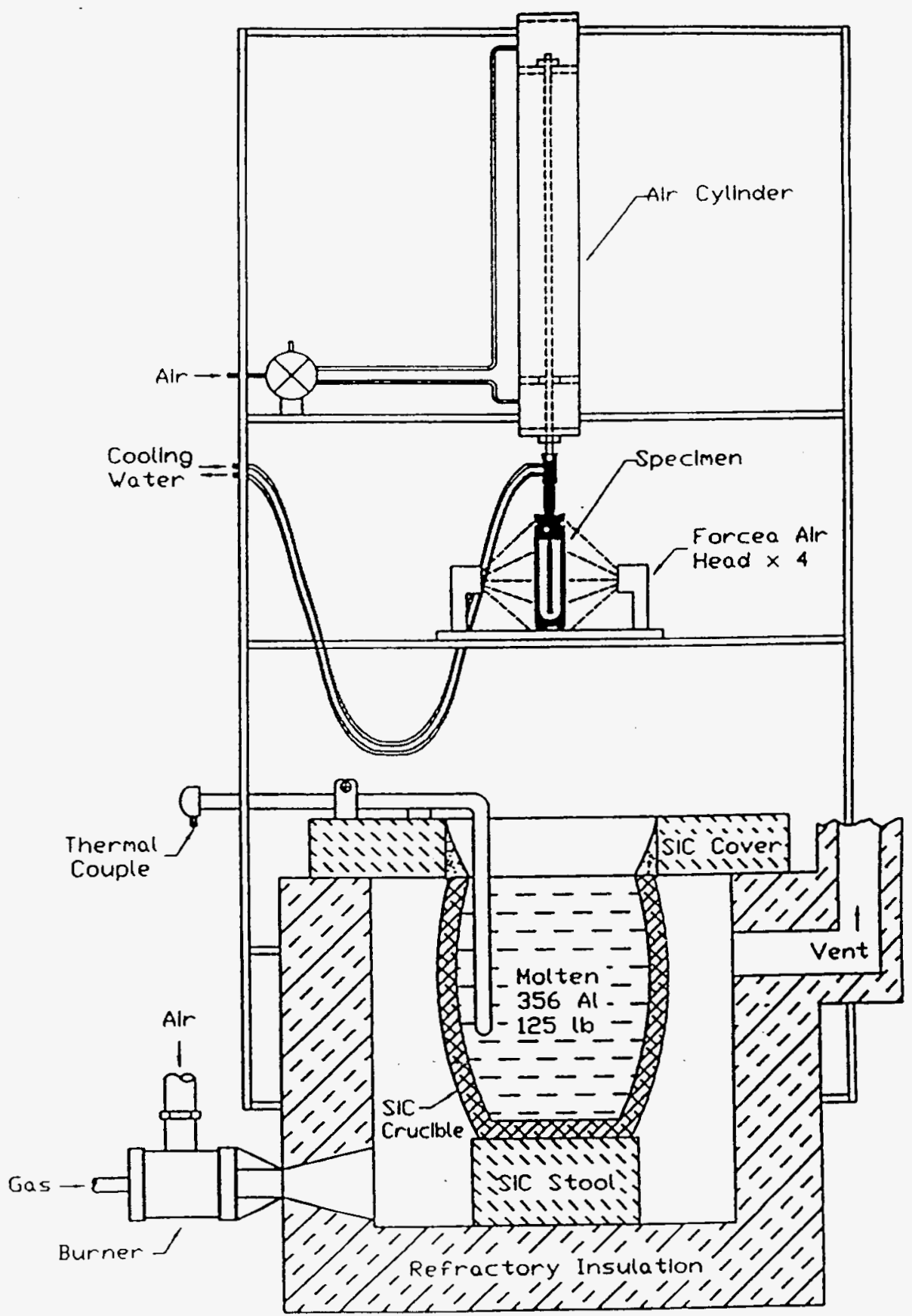

Figure 6 , Thermal test specimen and equipment for the coating testing 


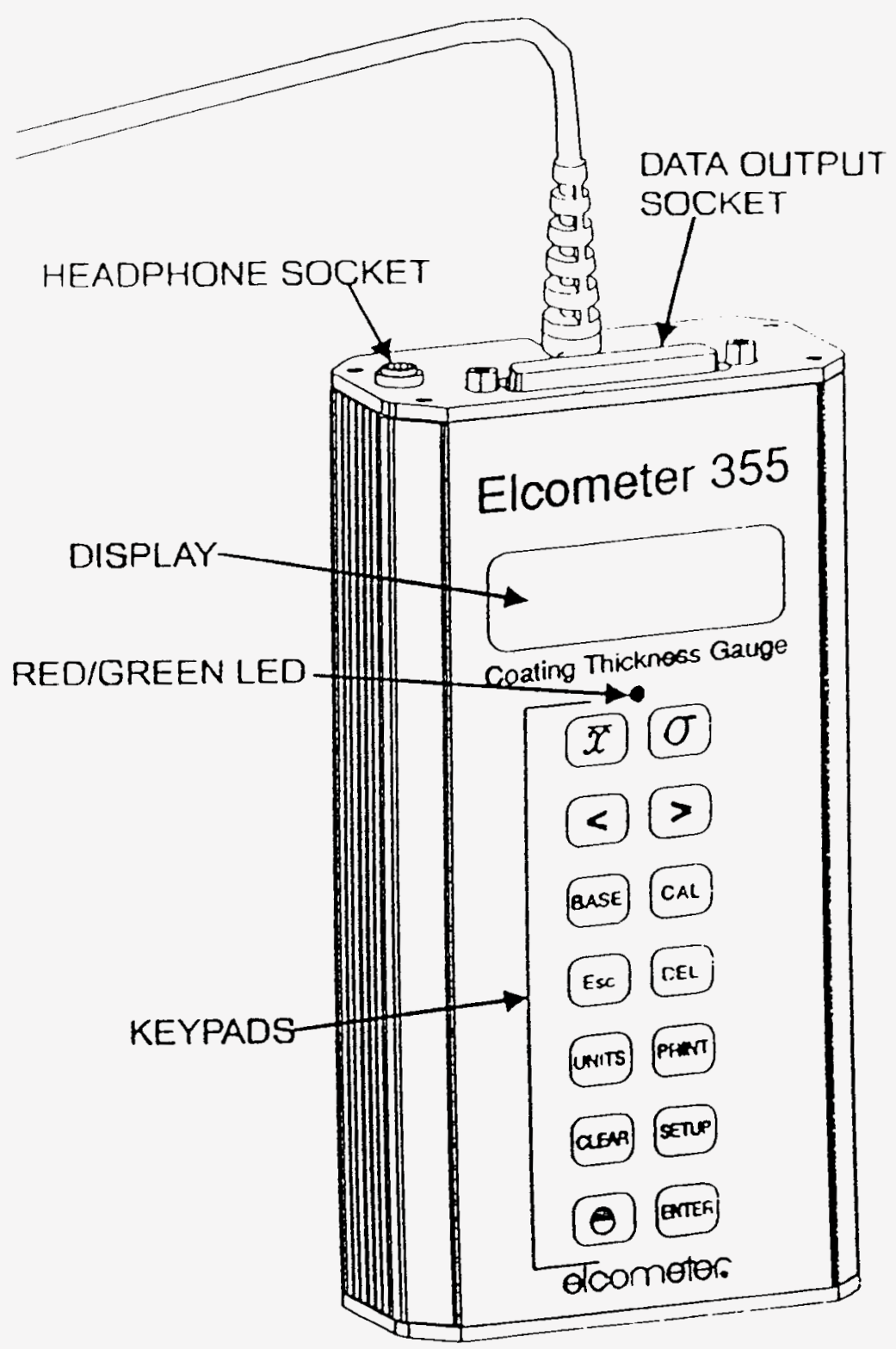

Figure 7, Sketch of Elcometer 355 
Figure 8 - Effect of Increasing Immersion Cycles on Maximum Temperature Difference Attaned During Immersion Cycles For Different Coatings

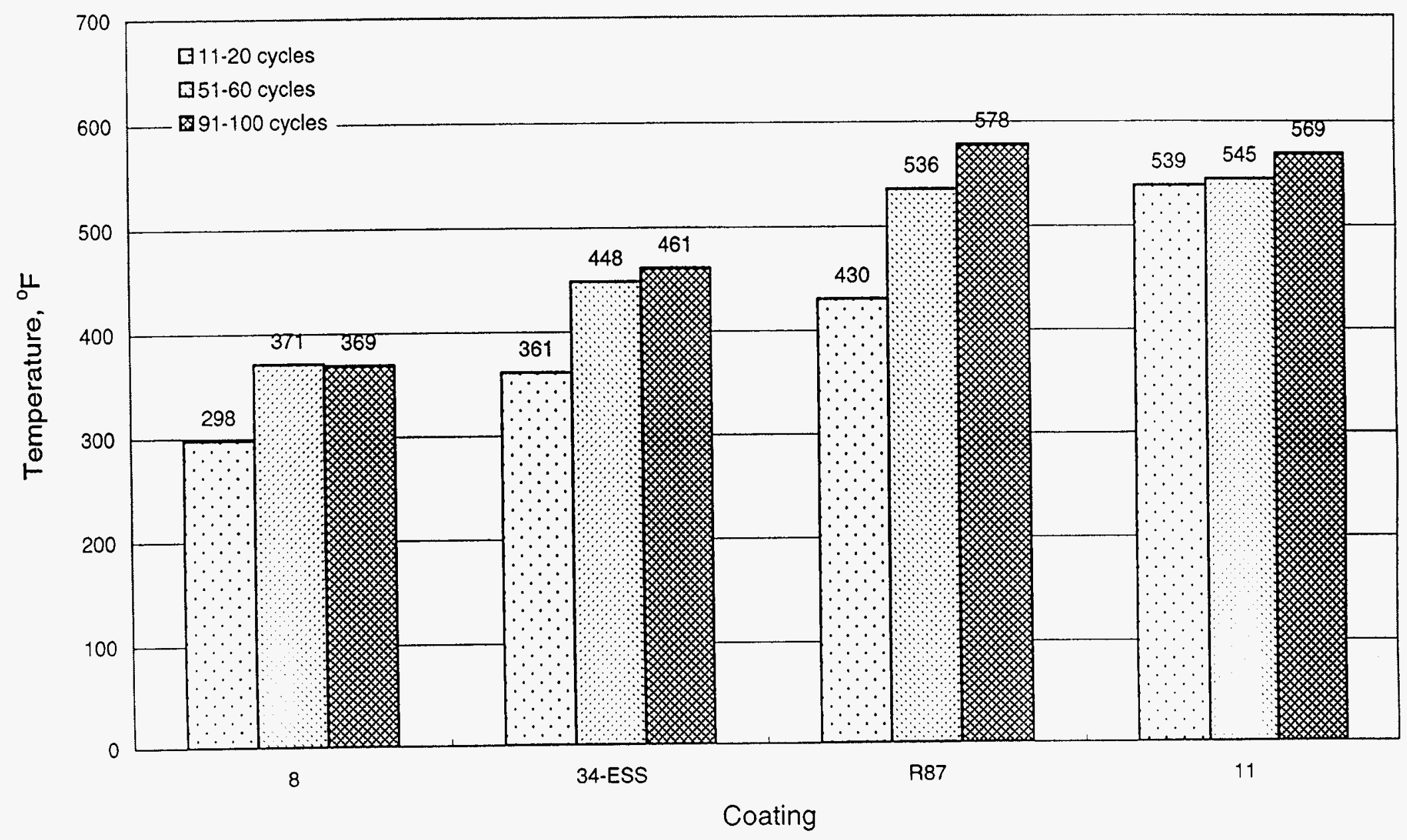


Figure 9 - Average Difference Between Maximum and Minimum Cycles Temperature in Coated Corner

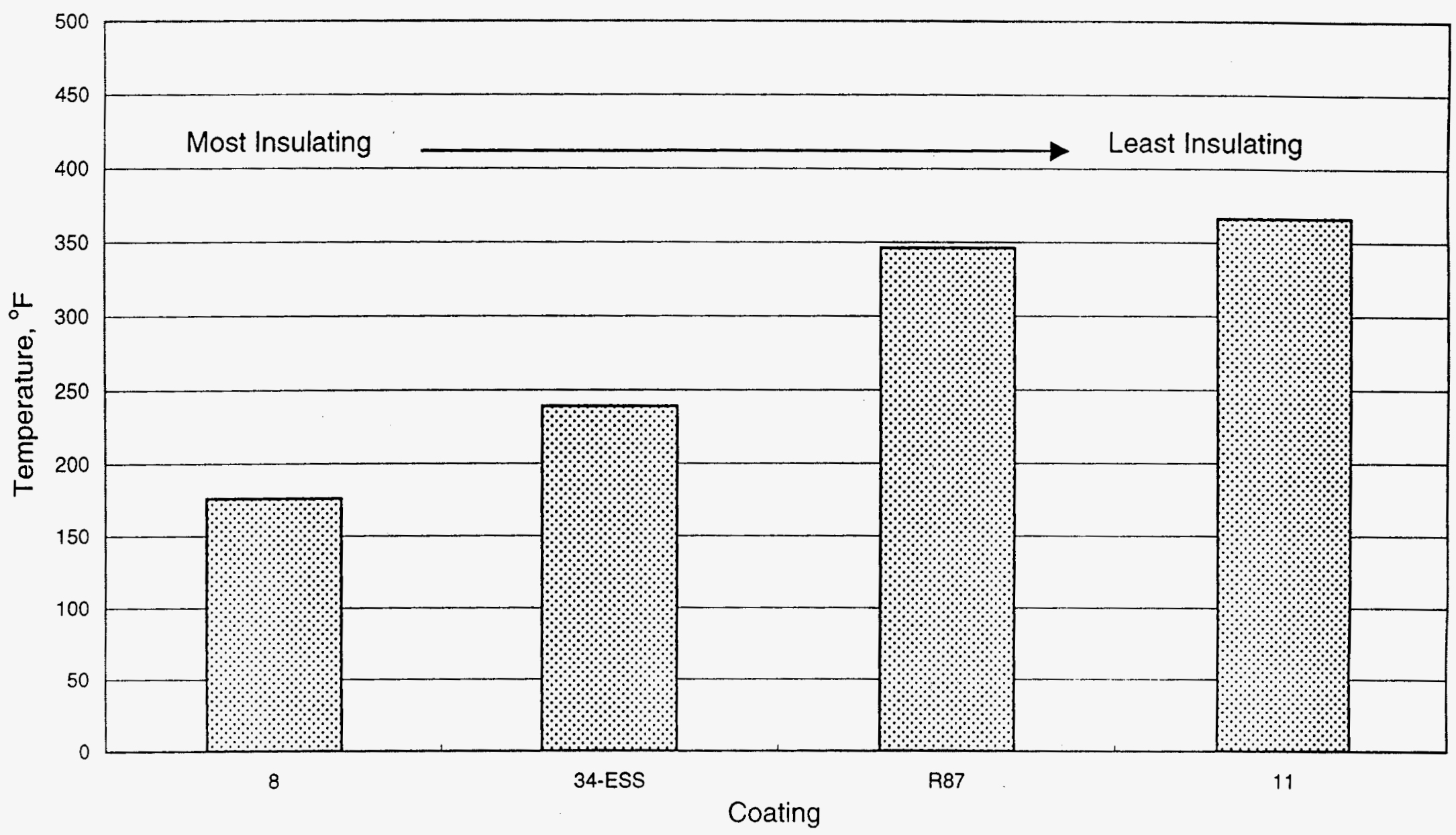




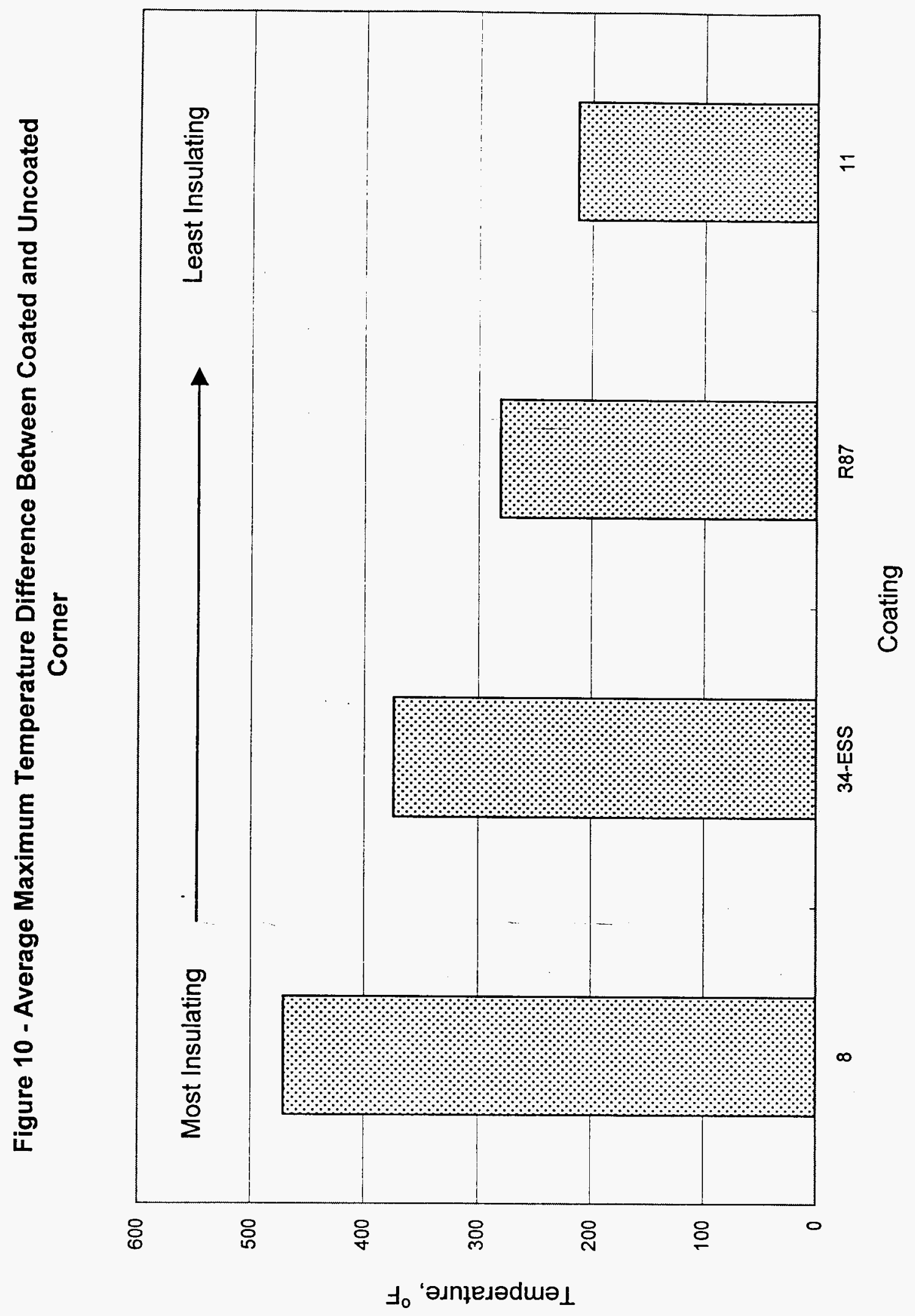




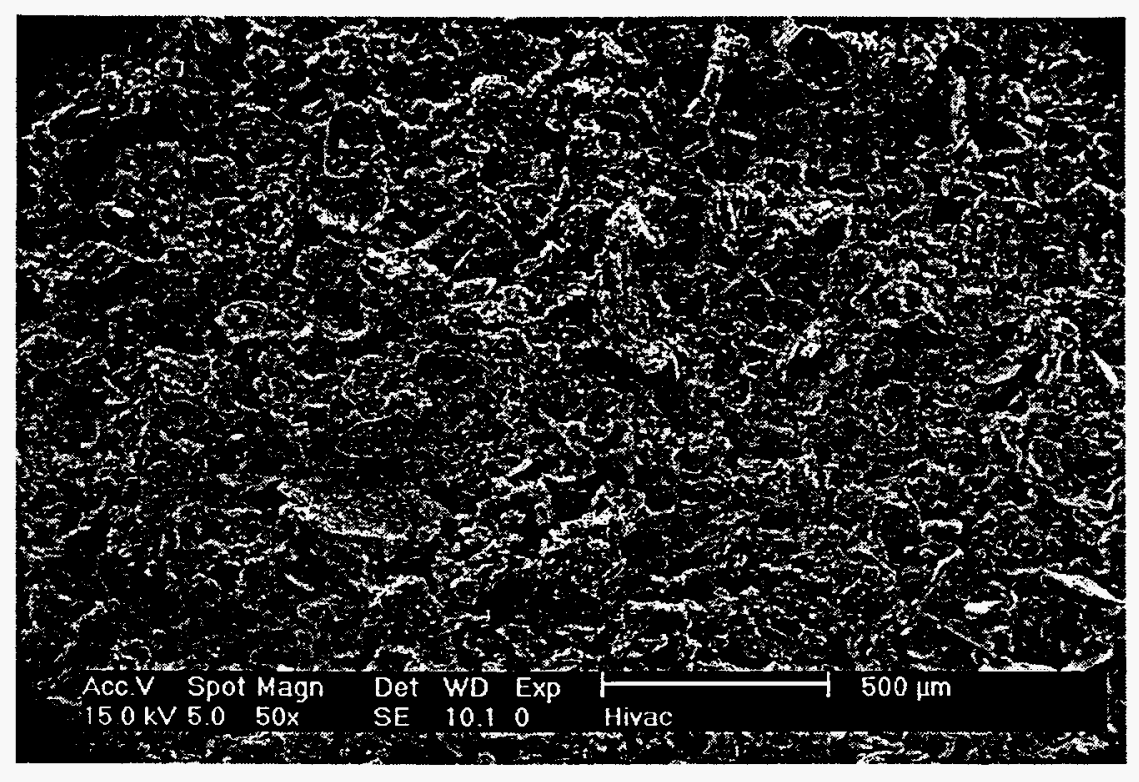

Figure 11(a)

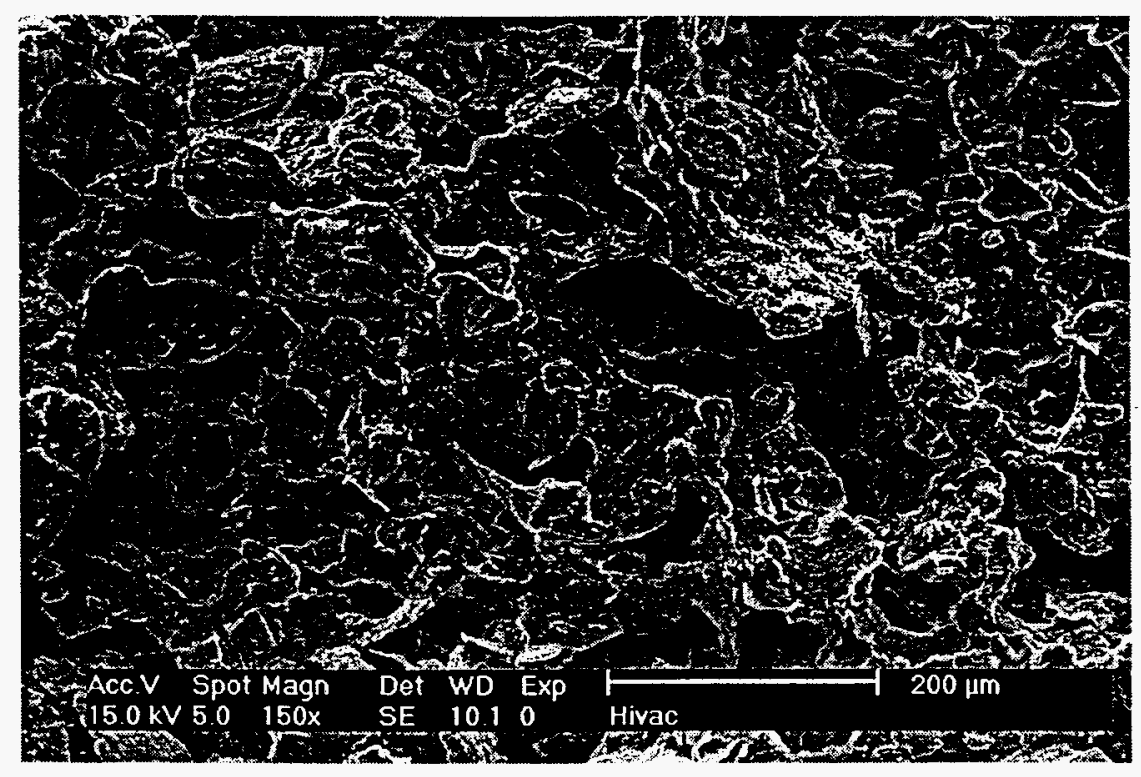

Figure 11(b) 


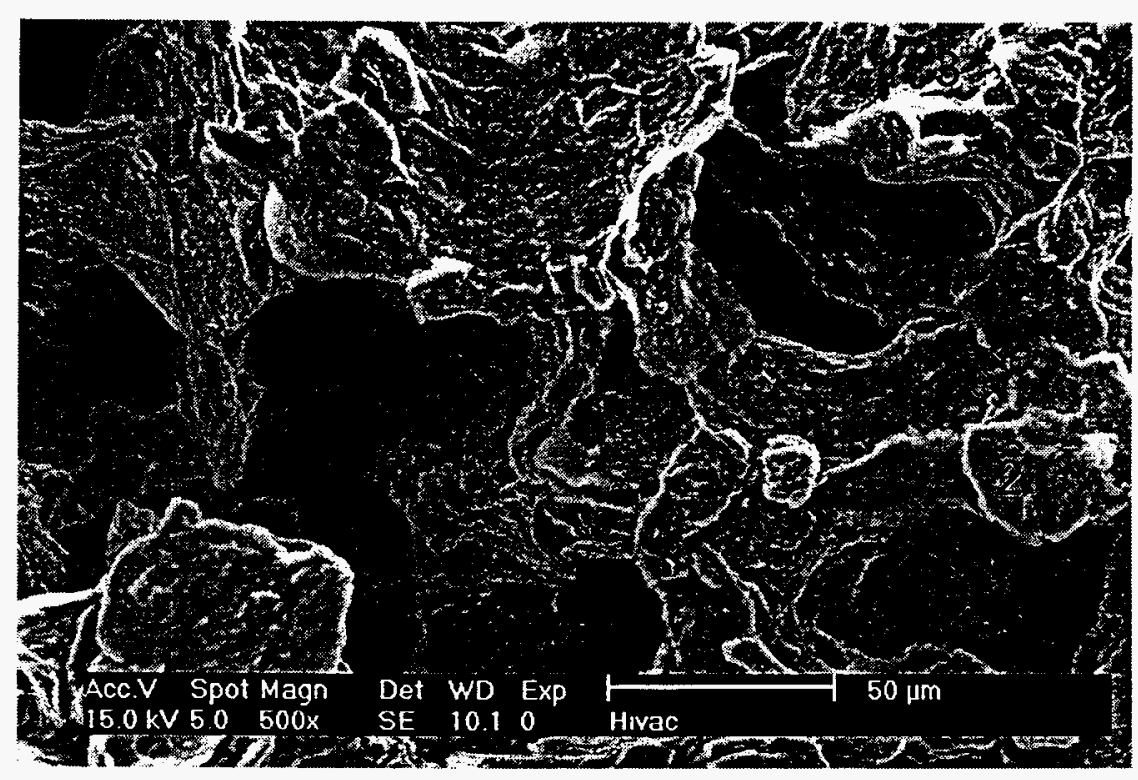

Figure 11(c)

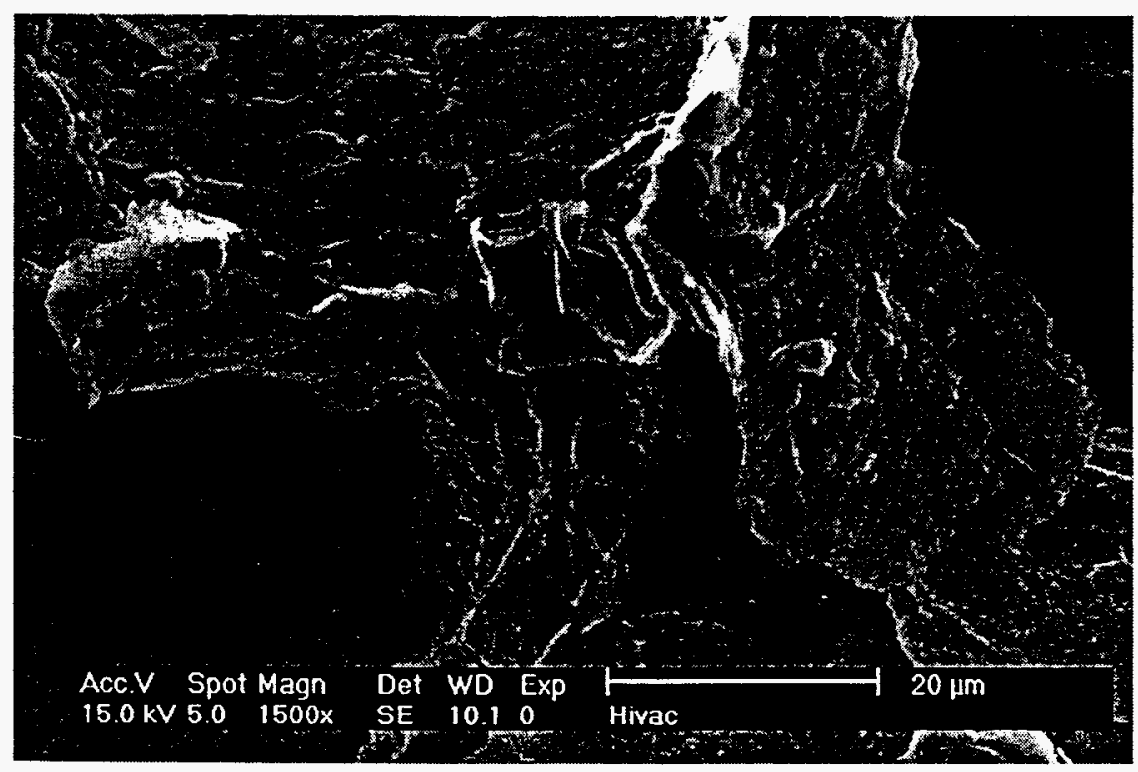

Figure 11(d)

Figure 11 -Morphology of Dycote 8 at Different Magnifications 


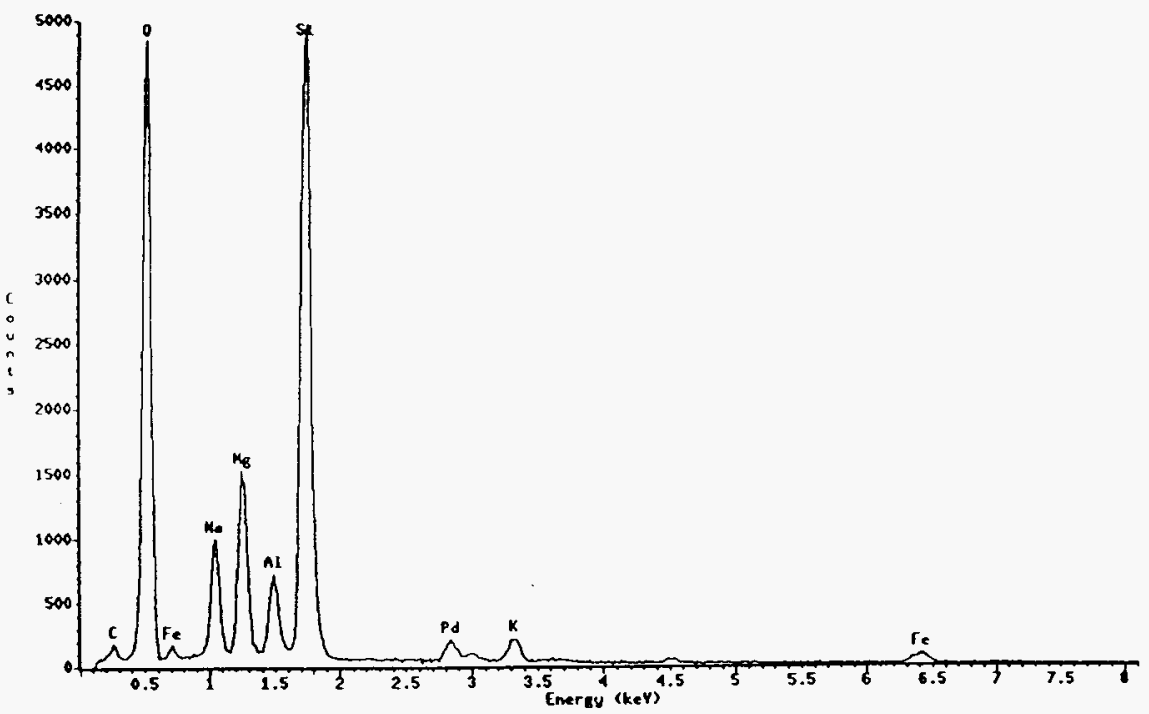

Figure 12(a) - EDS of Dycote 8 for Area 1 and Area 2

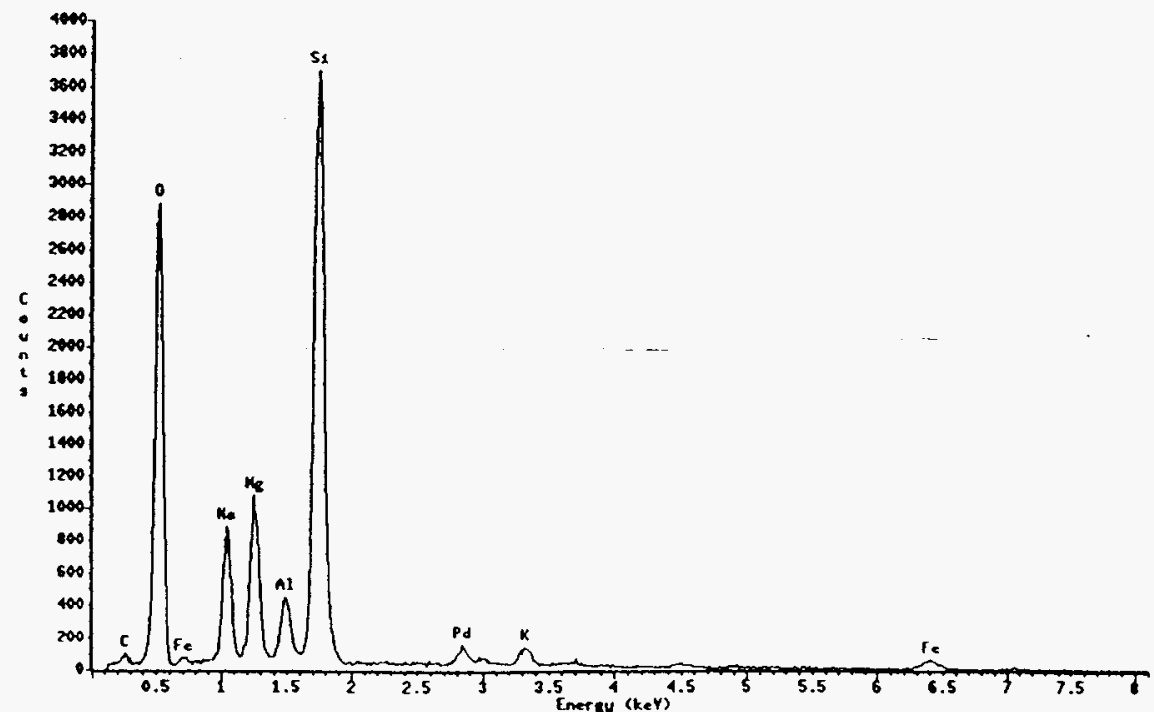

Figure 12(b) - EDS of Dycote 8 for Area 3 


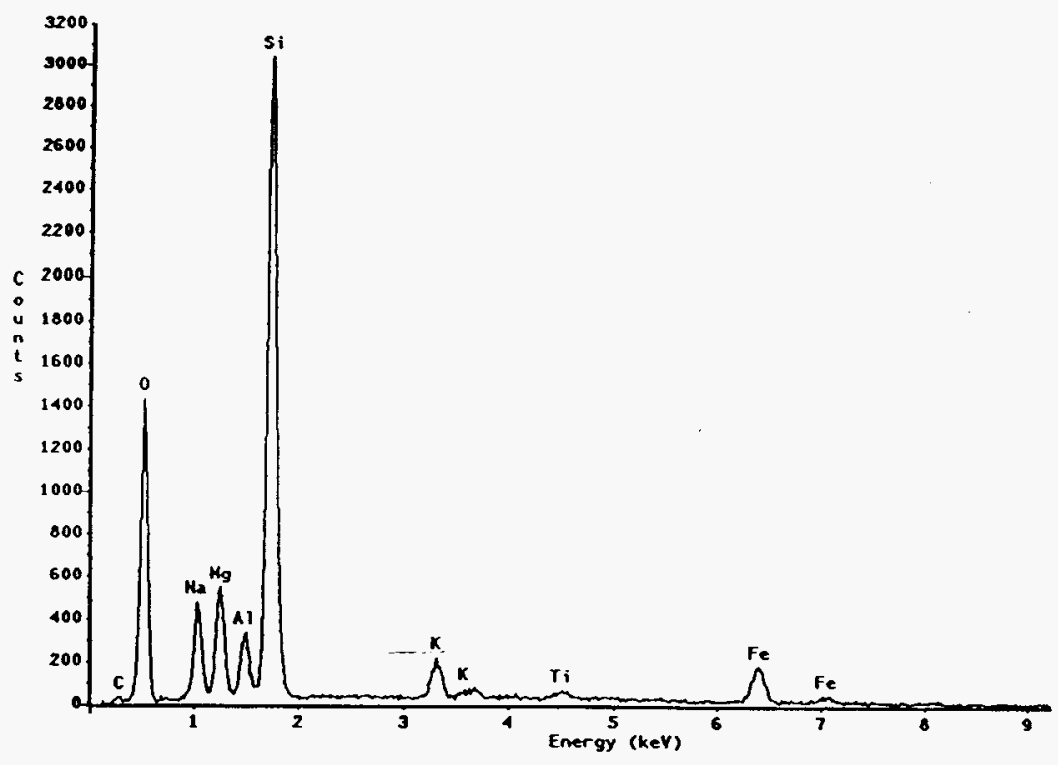

Figure 13 - The Overview EDS for Coating Dycote 8

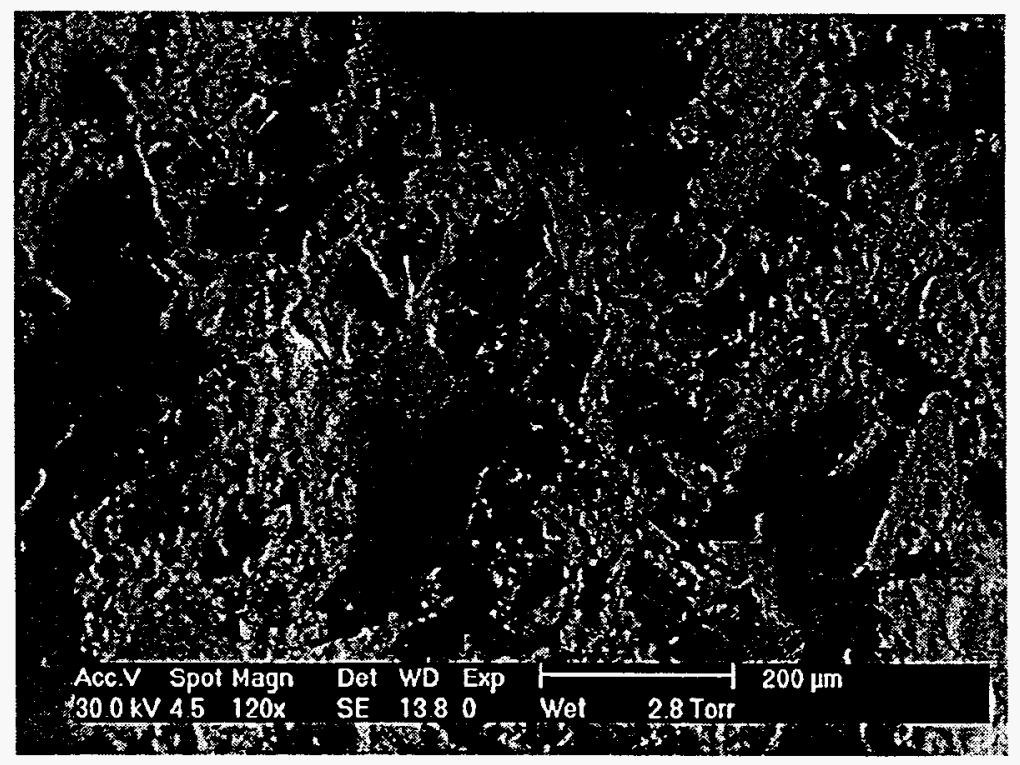

Figure 14 - Morphology of Dycote 8 With Oxide Particles 


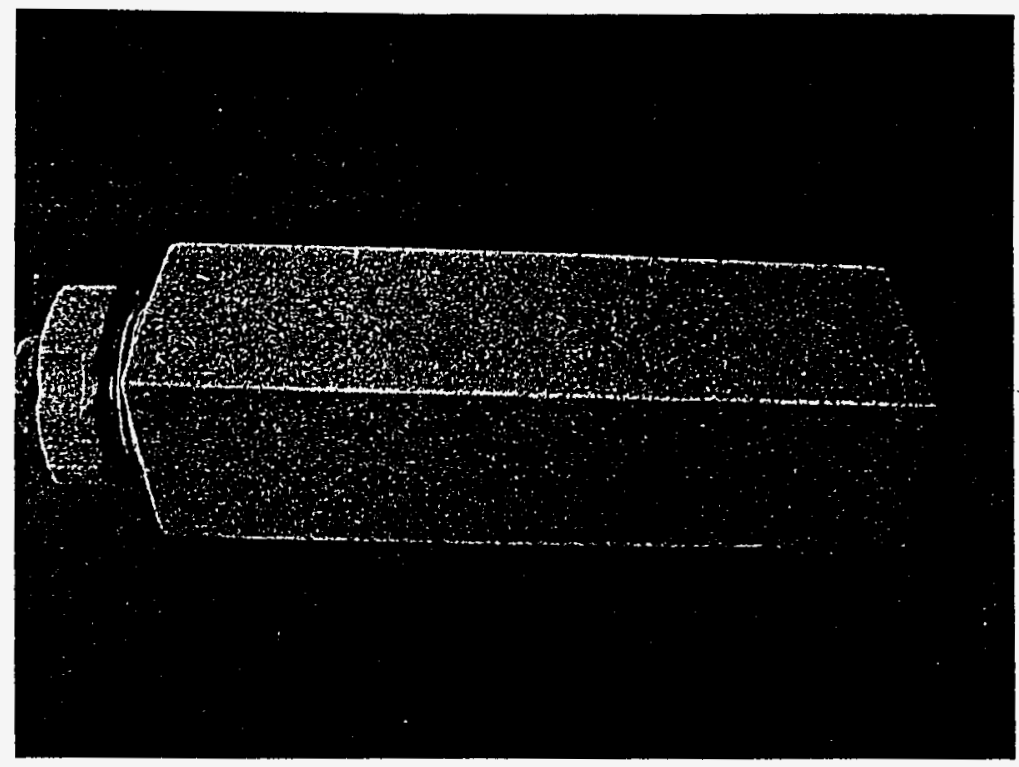

Figure 15 - Coated Corner with Dycote 8 before Cyclic Immersion

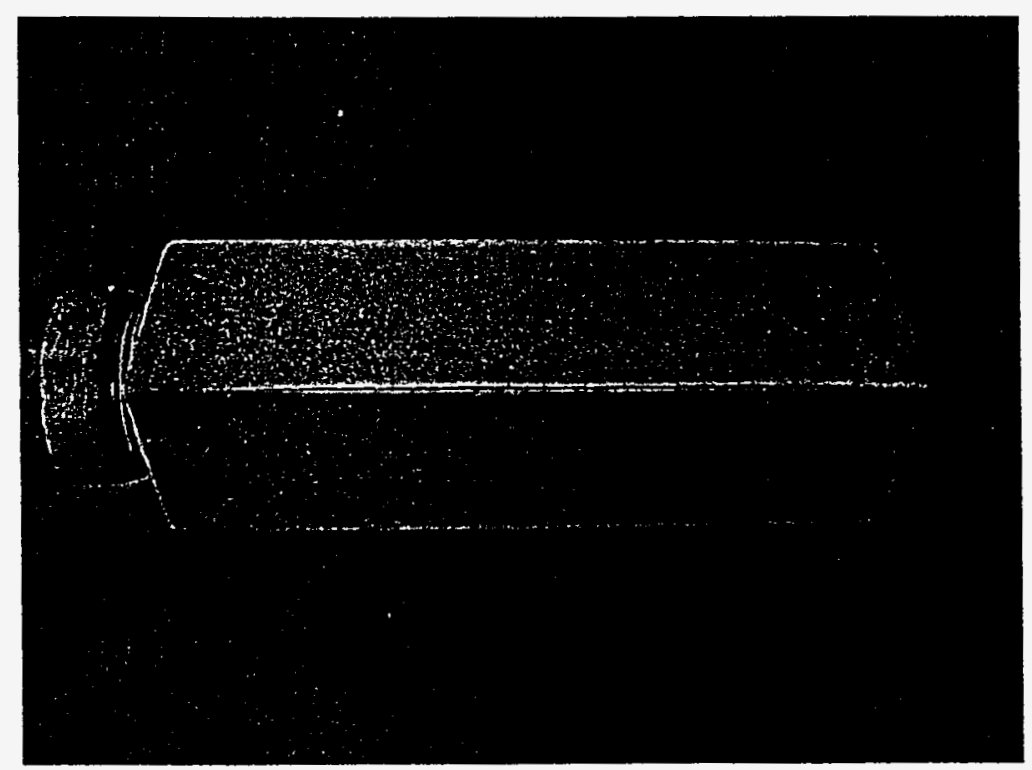

Figure 16 - Coated Corner with Dycote 8 after Cyclic Immersion 


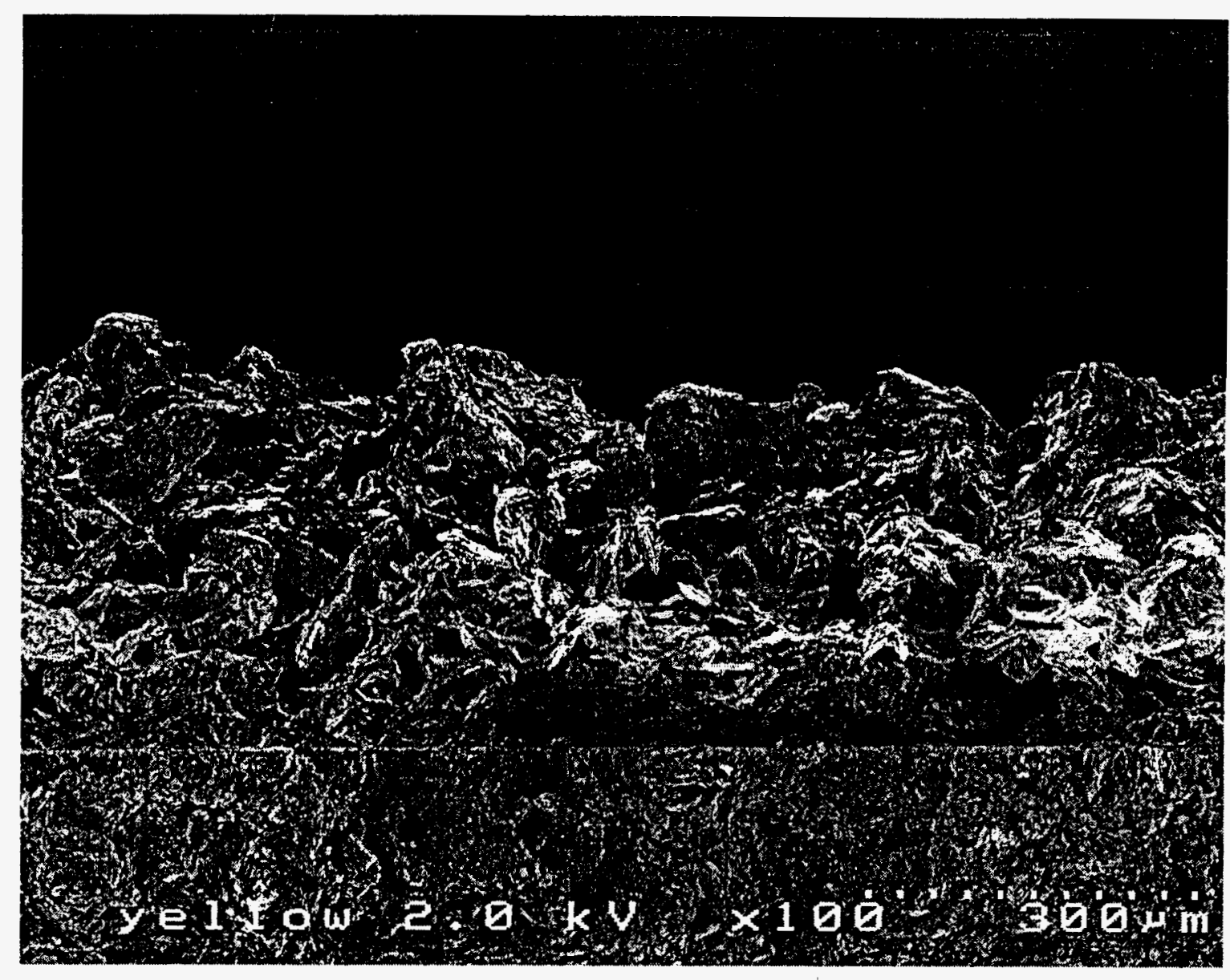

Figure 17 -Photograph of Cross-section for Coating Dycote 8 


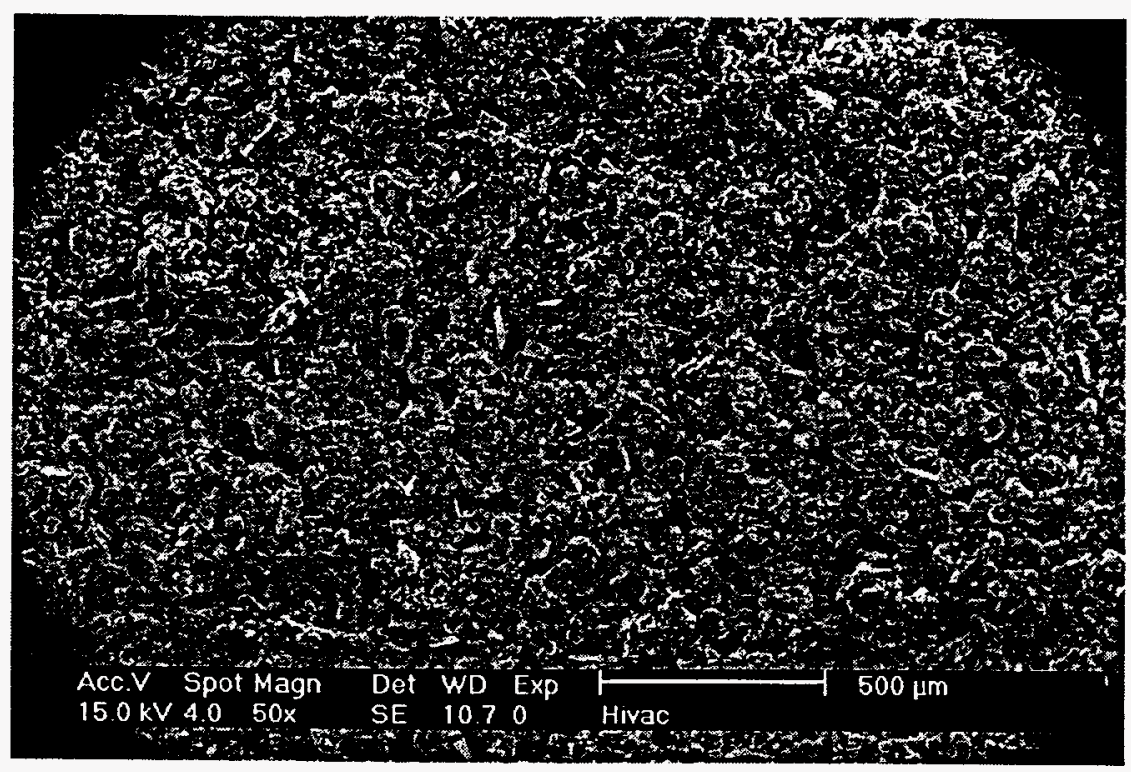

Figure 18(a)

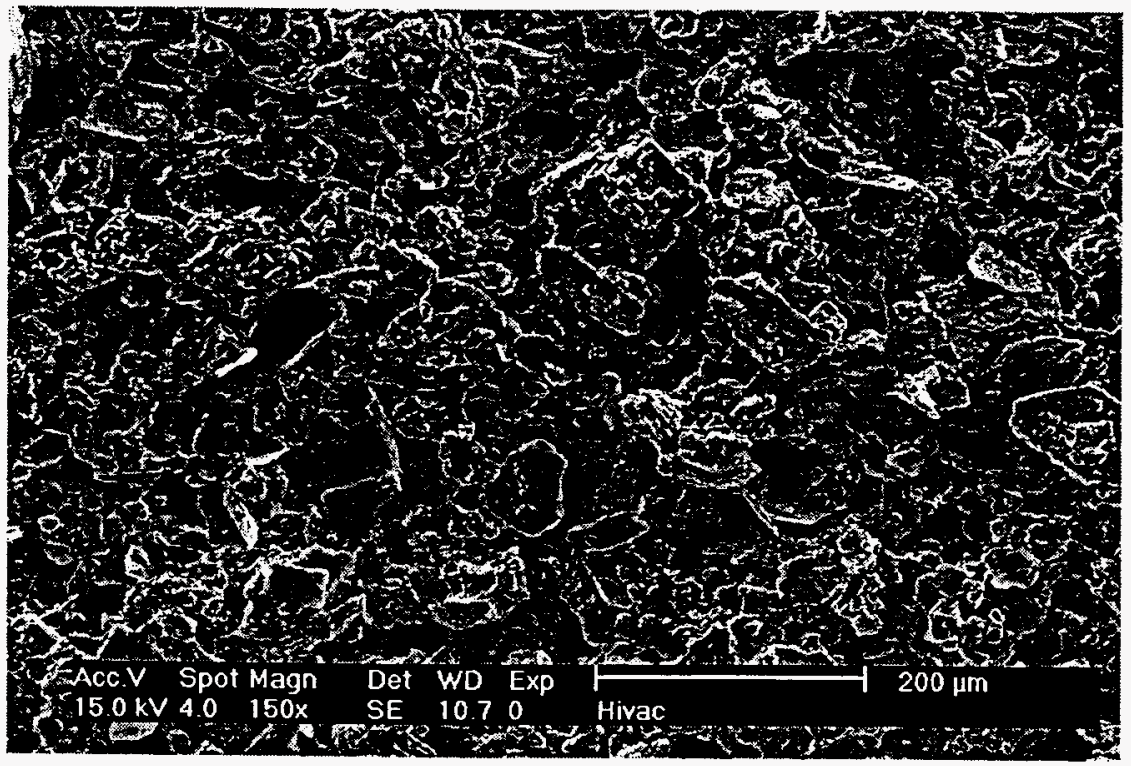

Figure 18(b) 


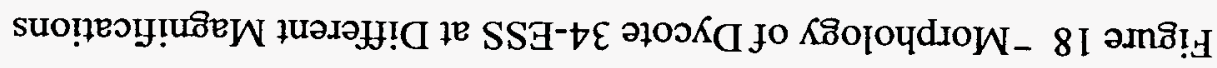

(p)8I ว.กชิ!

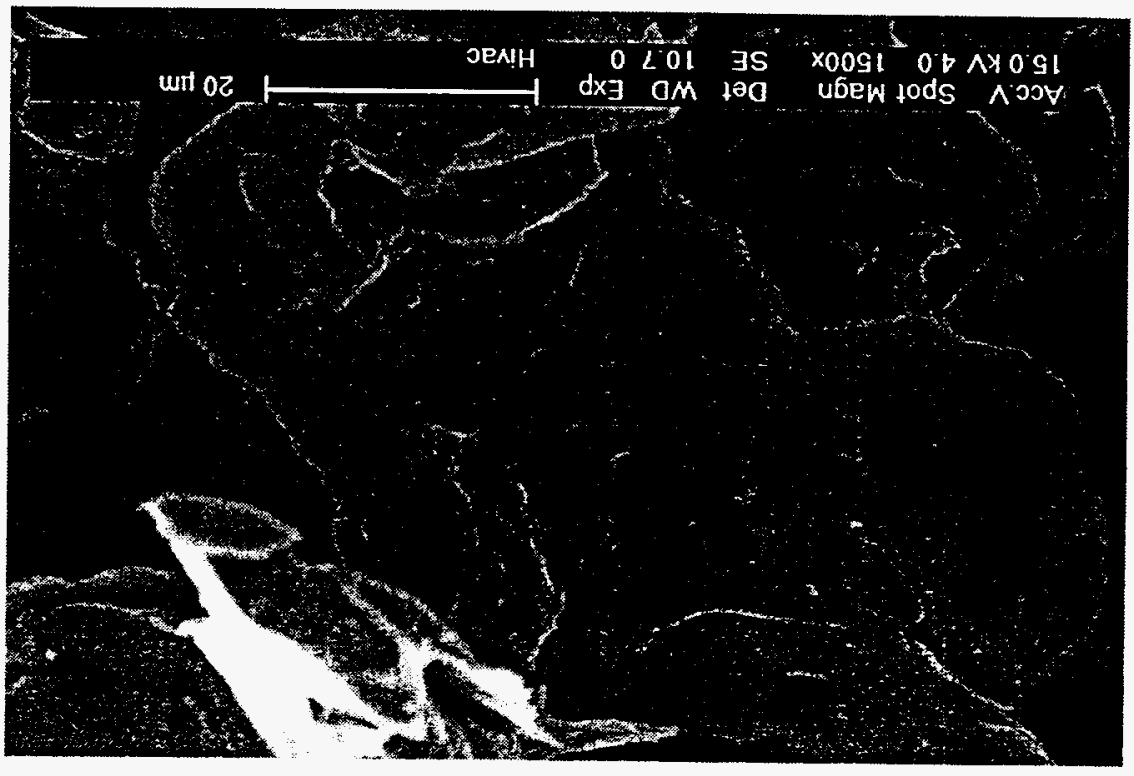

(ว) 8 I ว.mริ!ป

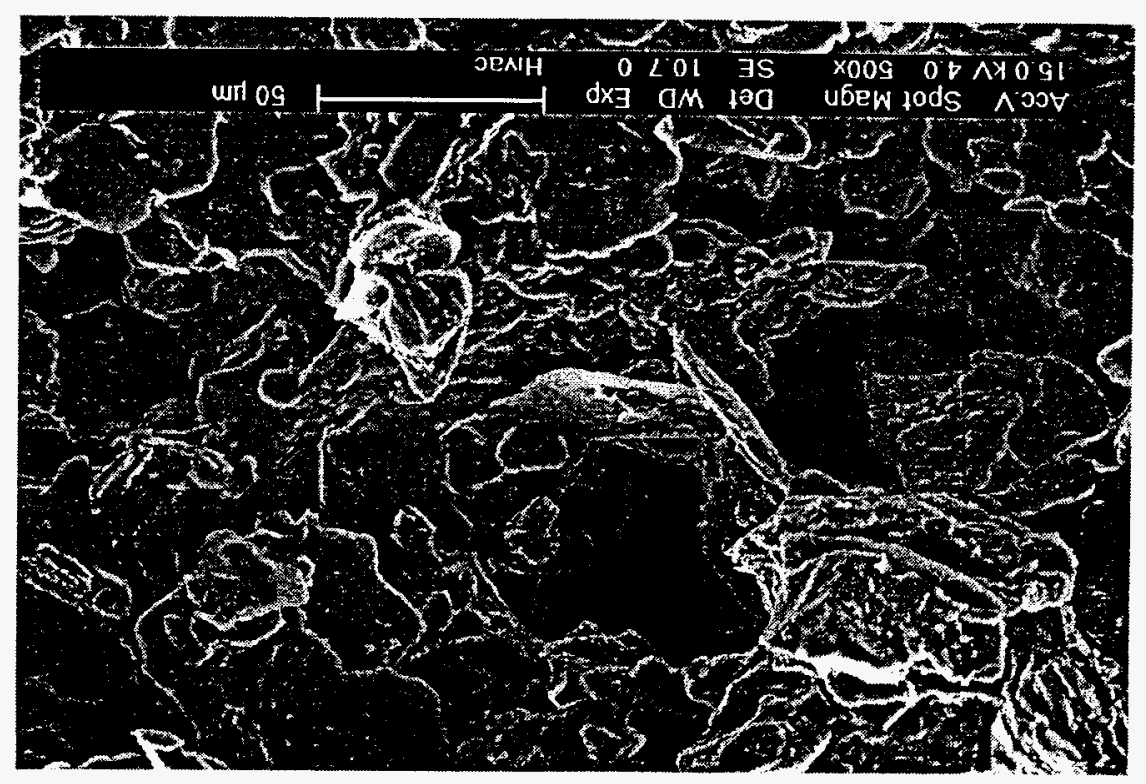




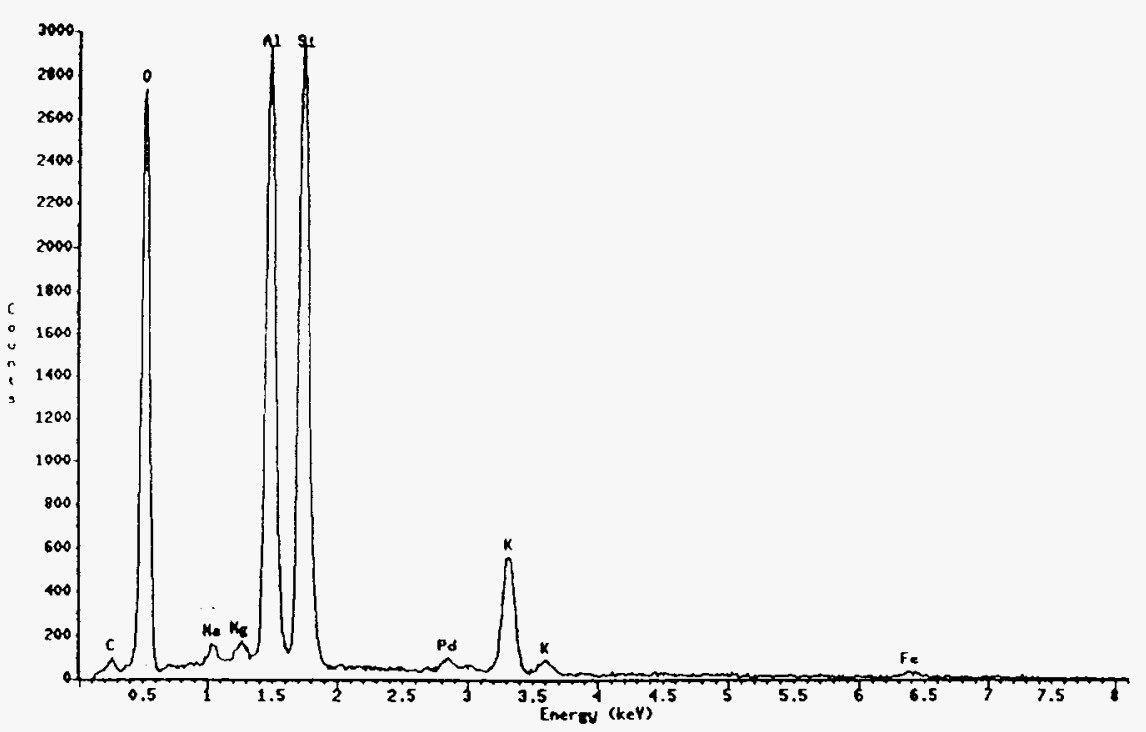

Figure 19 (a) - EDS of Dycote 34-ESS for Area 1

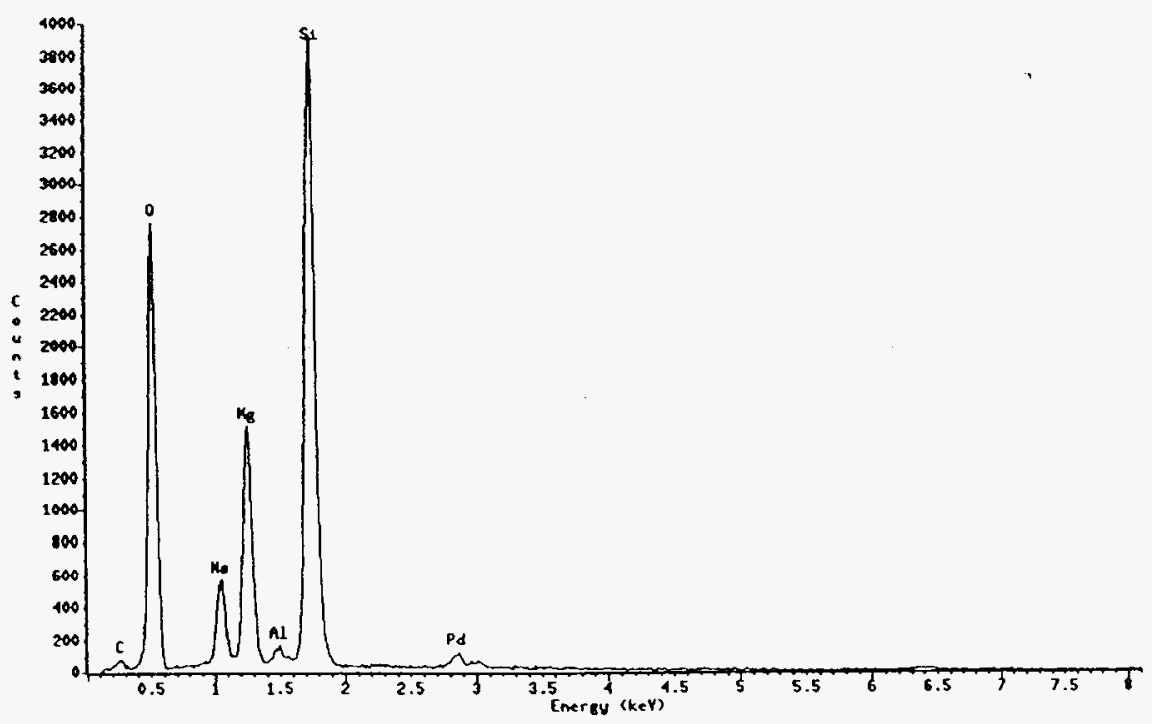

Figure 19 (b) - EDS of Dycote 34-ESS for Area 2 


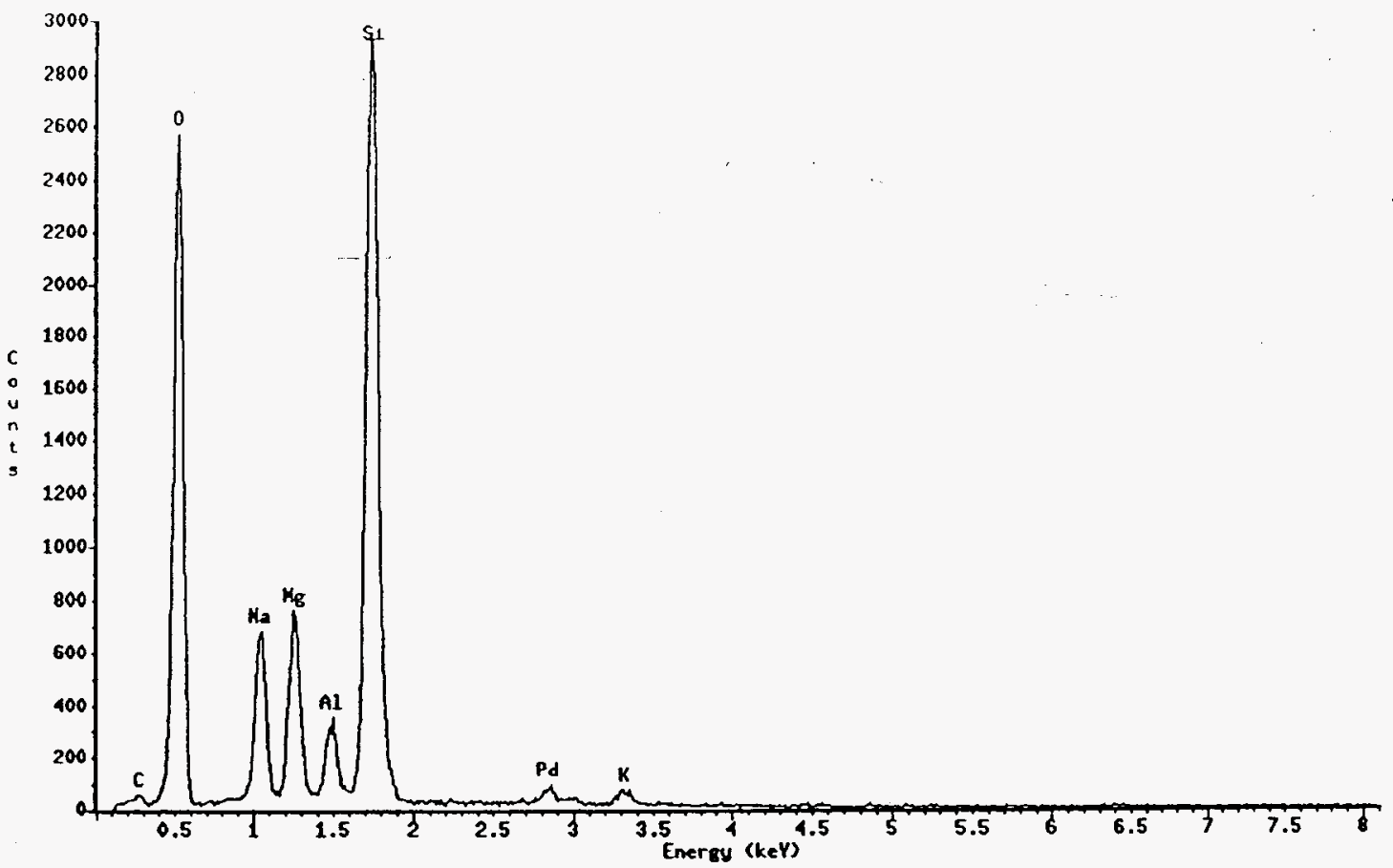

Figure 19 (c) - EDS of Dycote 34-ESS for Area 3 


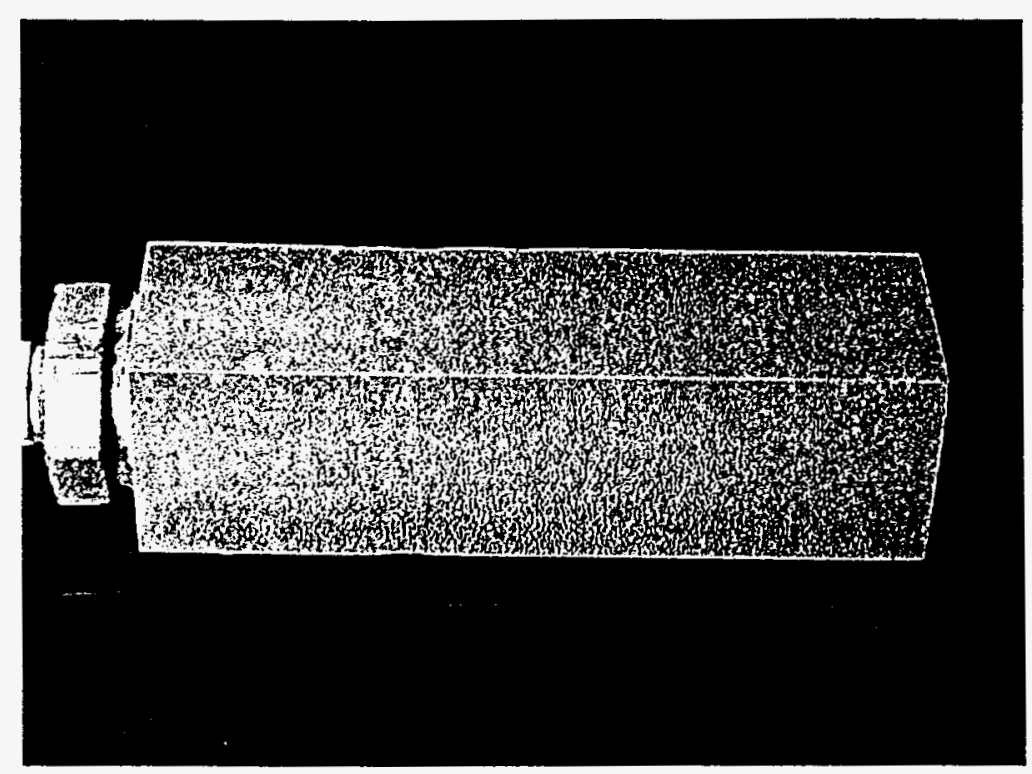

Figure 20 - Coated Corner with Dycote 34-ESS before Cyclic Immersion

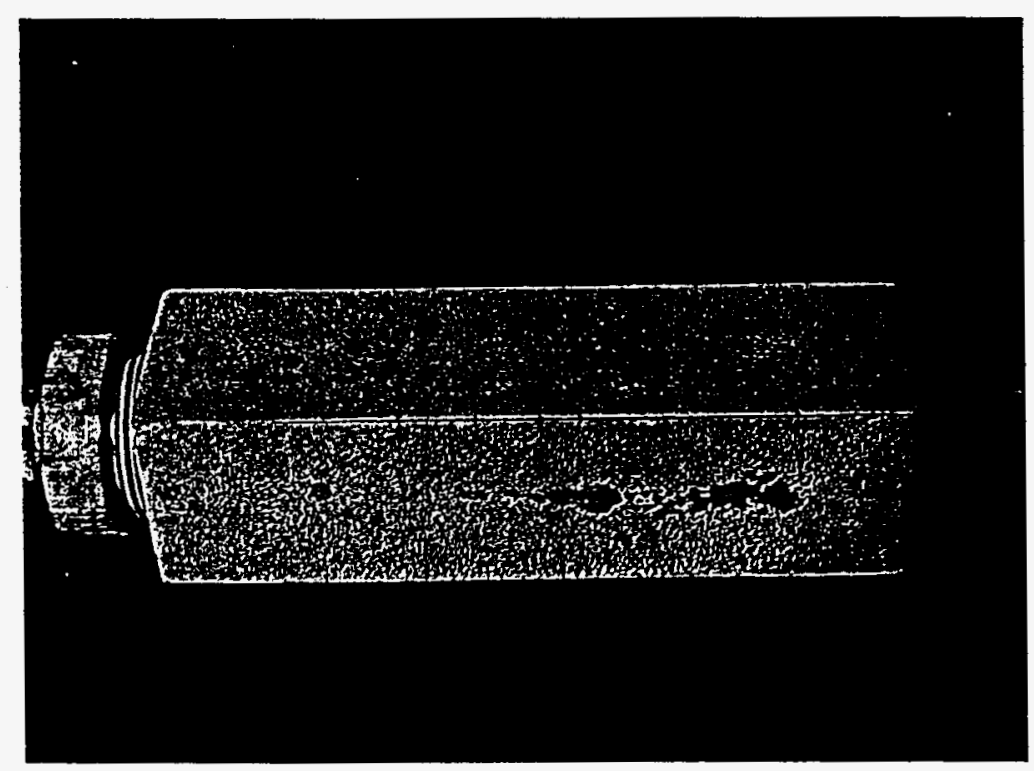

Figure 21 - Coated Corner with Dycote 34-ESS after Cyclic Immersion 


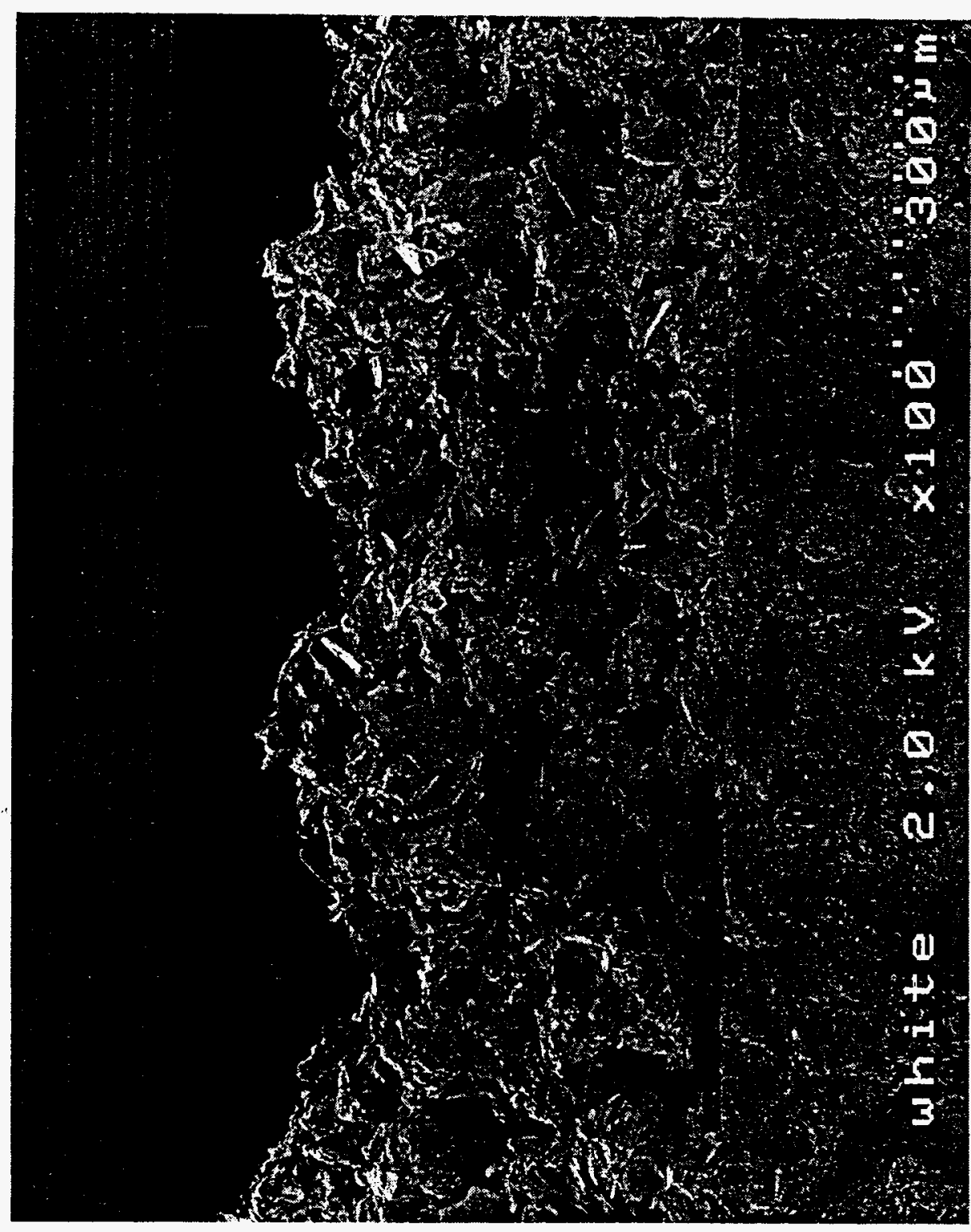

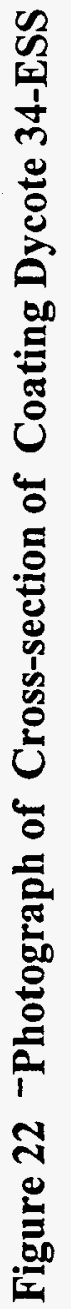




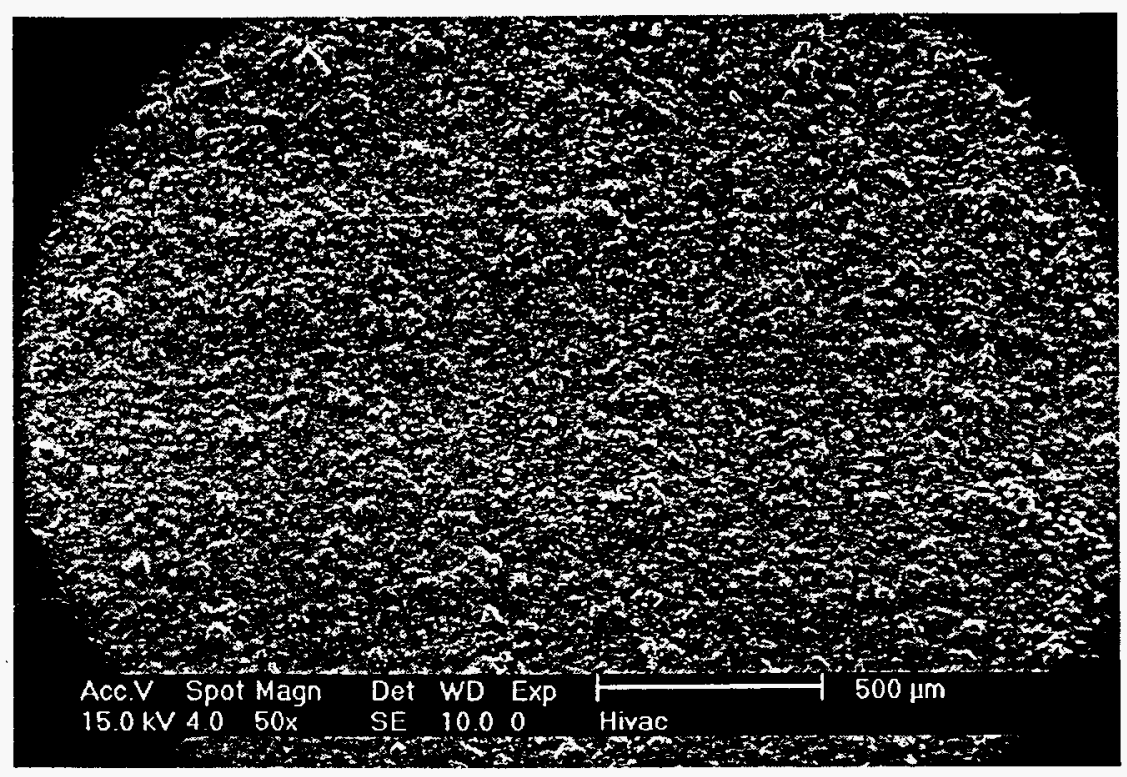

Figure 23(a)

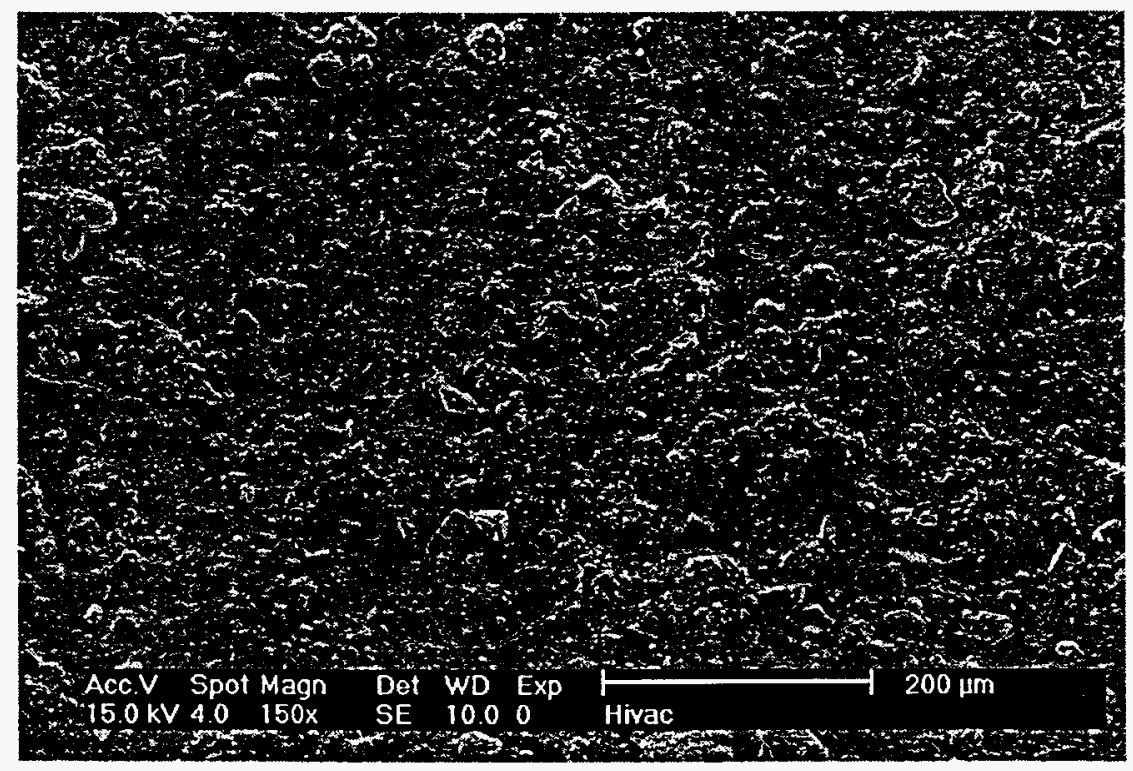

Figure 23(b) 


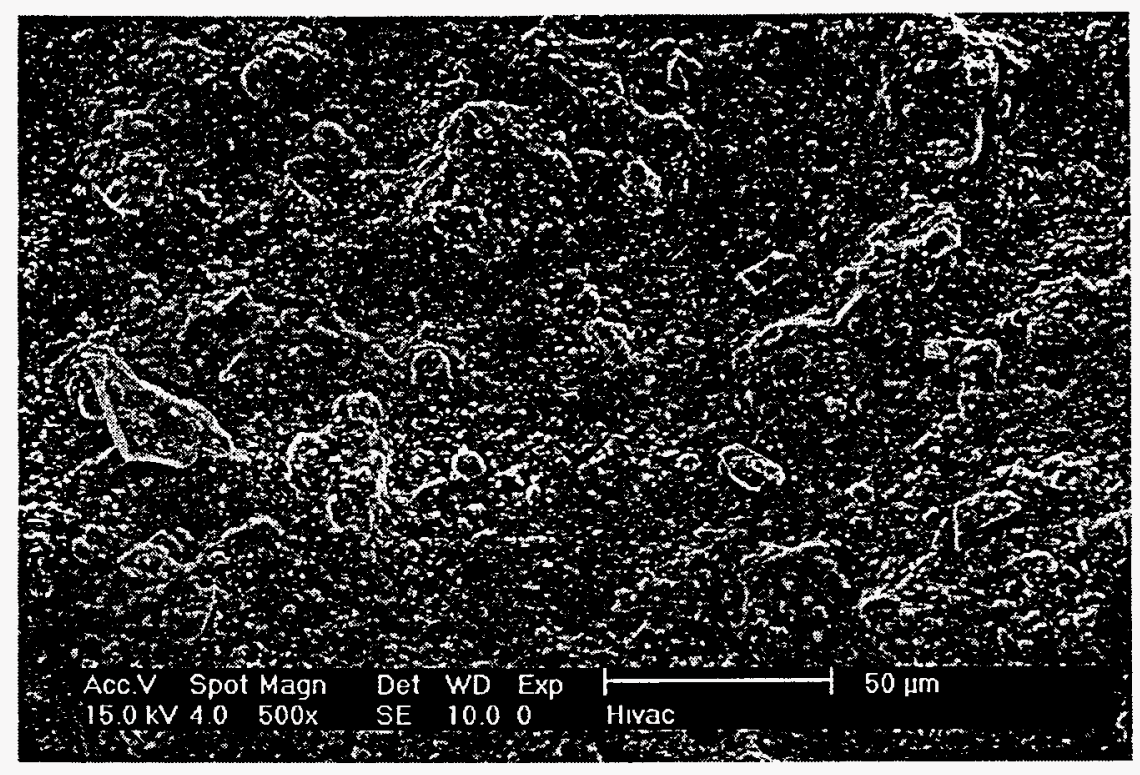

Figure 23(c)

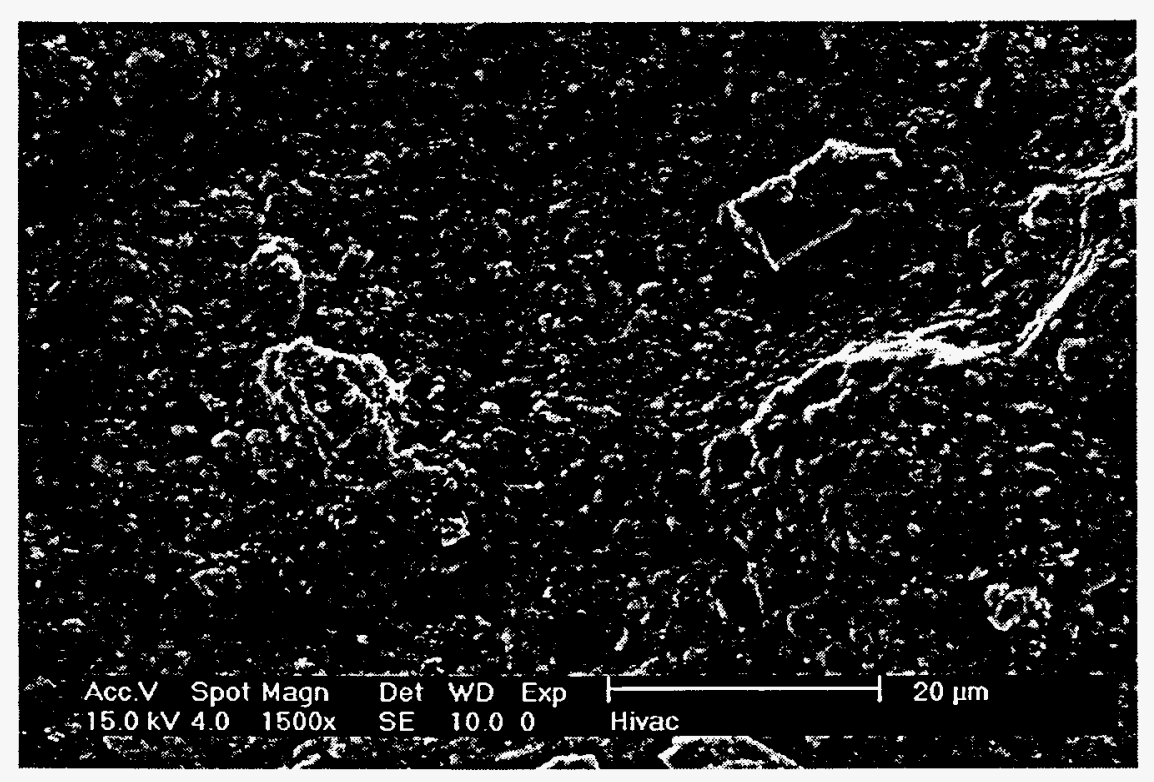

Figure 23(d)

Figure 23 - Morphology of Dycote R87 at Different Magnifications 


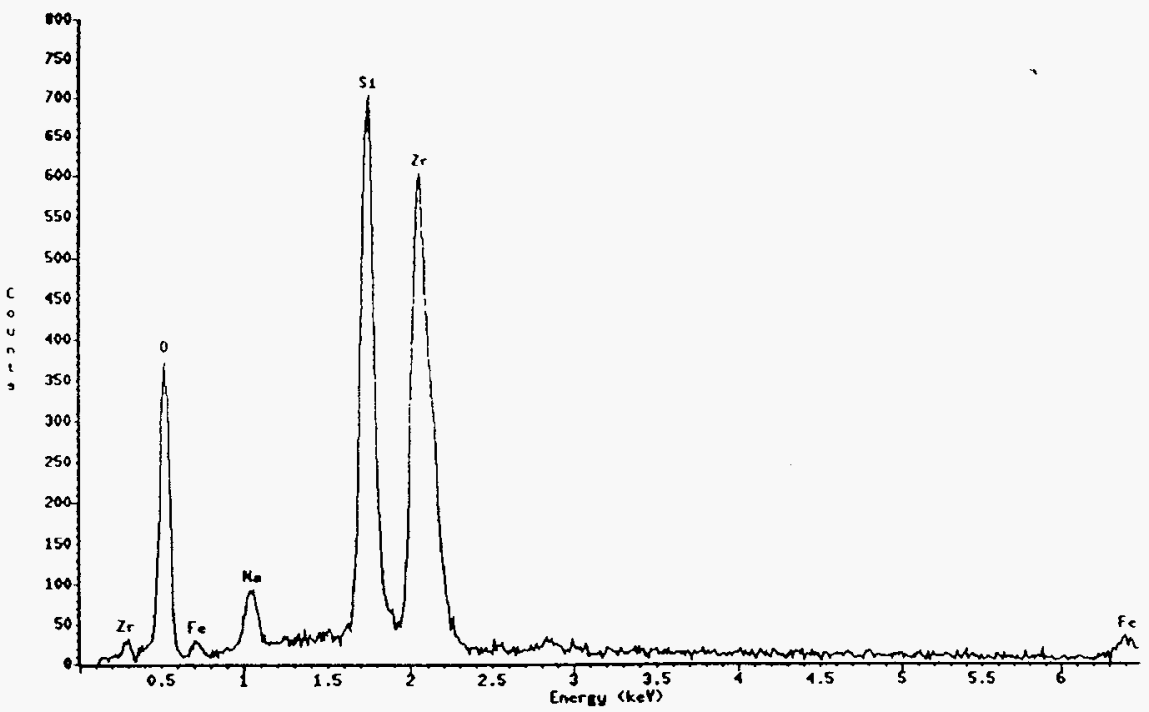

Figure 24 (a) - EDS of Dycote R87 for Area 1

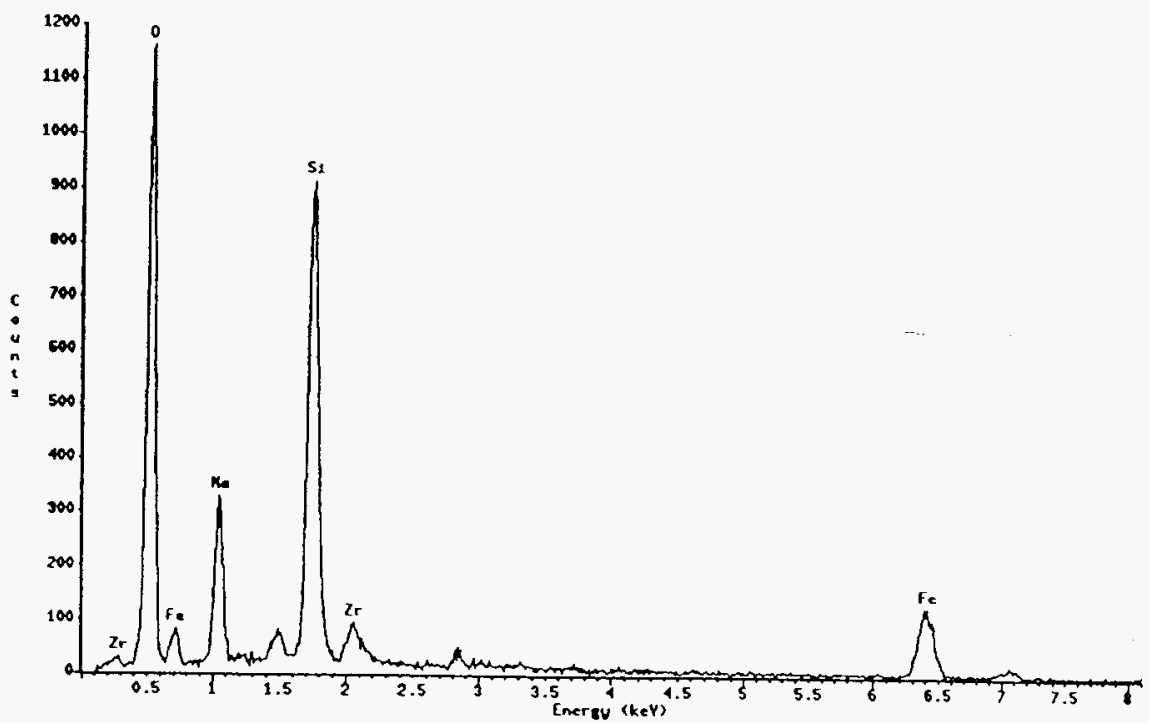

Figure 24 (b) - EDS of Dycote R87 for Area 2 


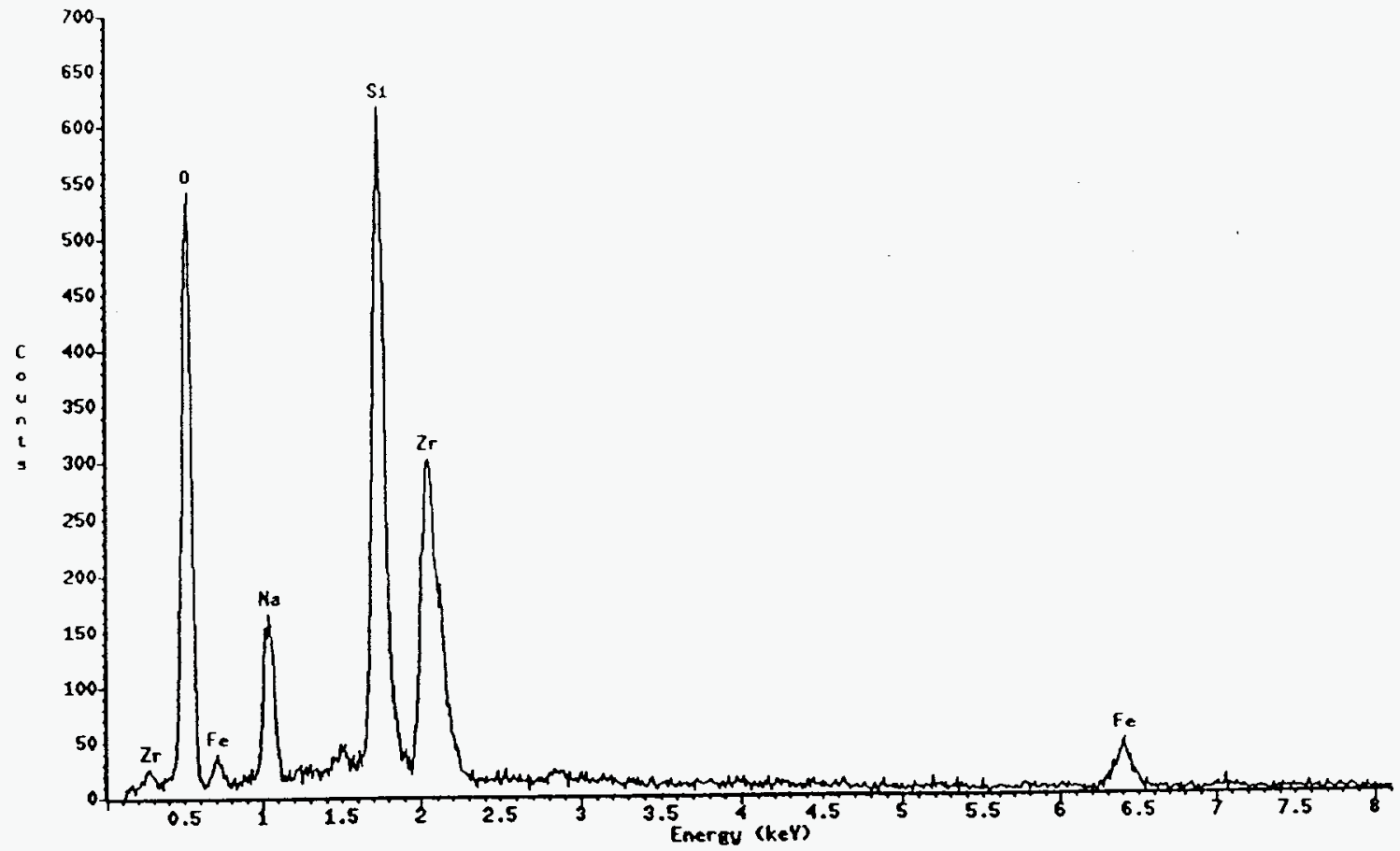

Figure 24 (c) - EDS of Dycote R87 for Area 3 


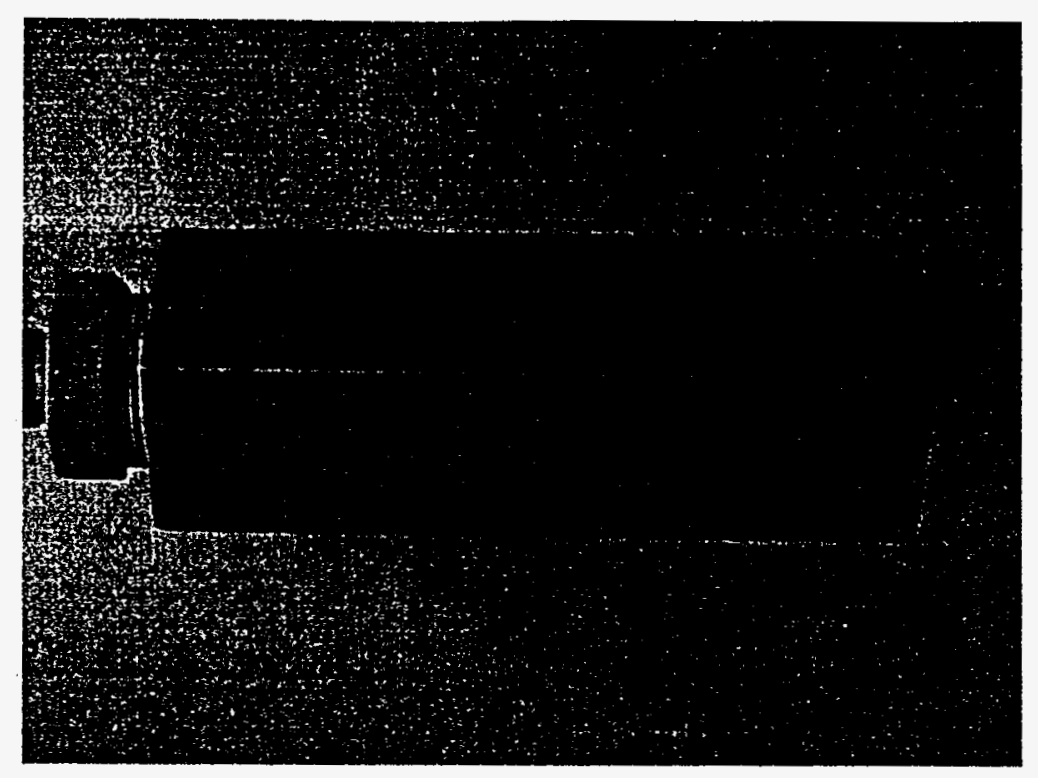

Figure 25 - Coated Corner with Dycote R87 before Cyclic Immersion

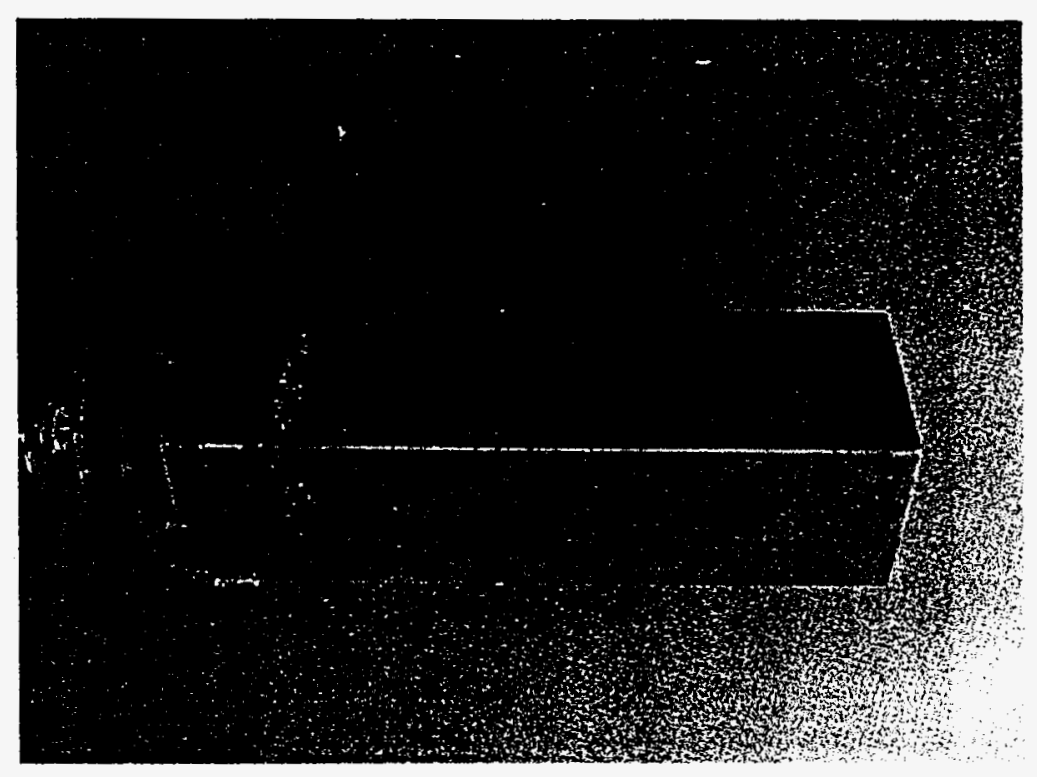

Figure 26 - Coated Corner with Dycote R87 after Cyclic Immersion 


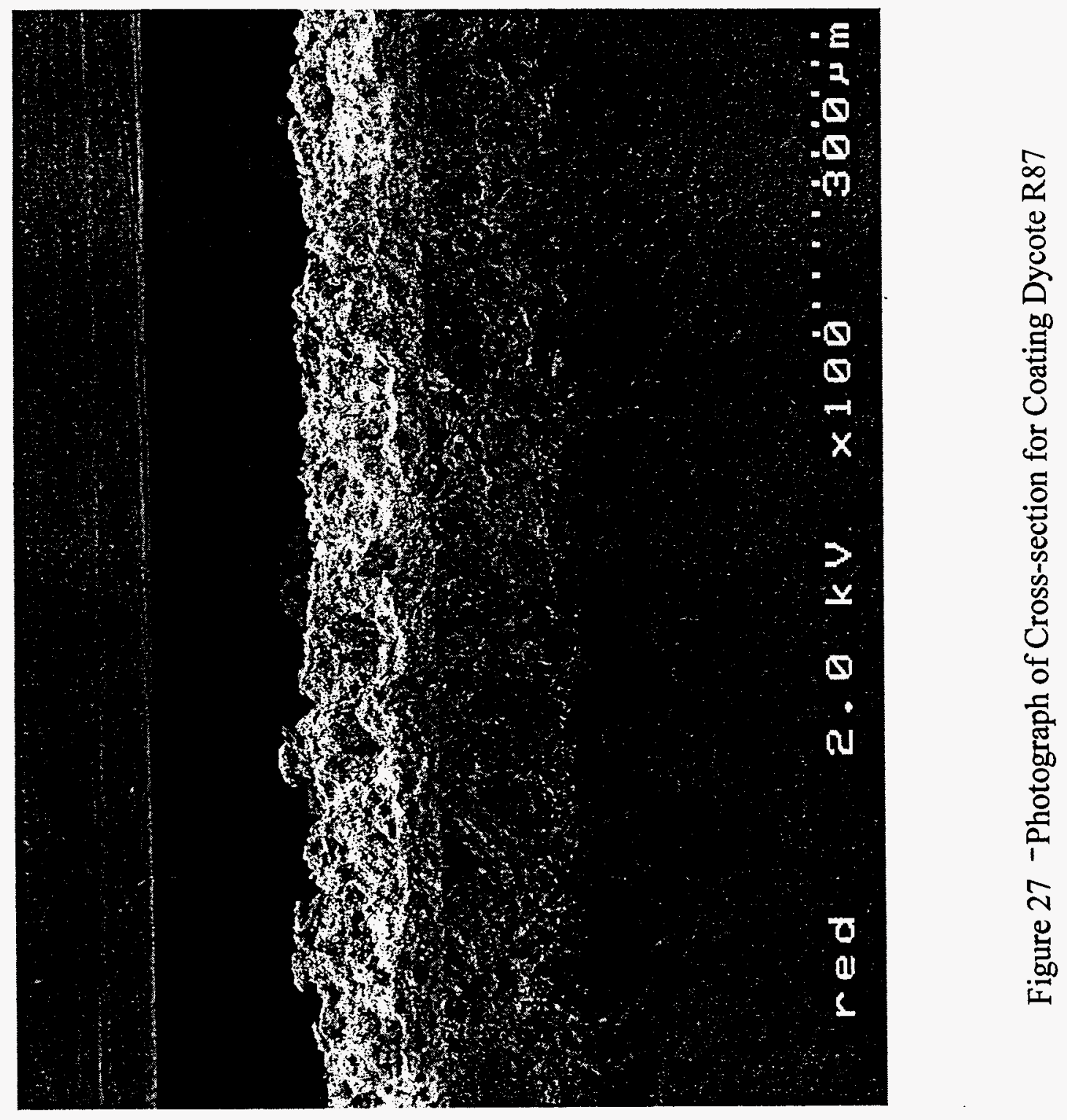




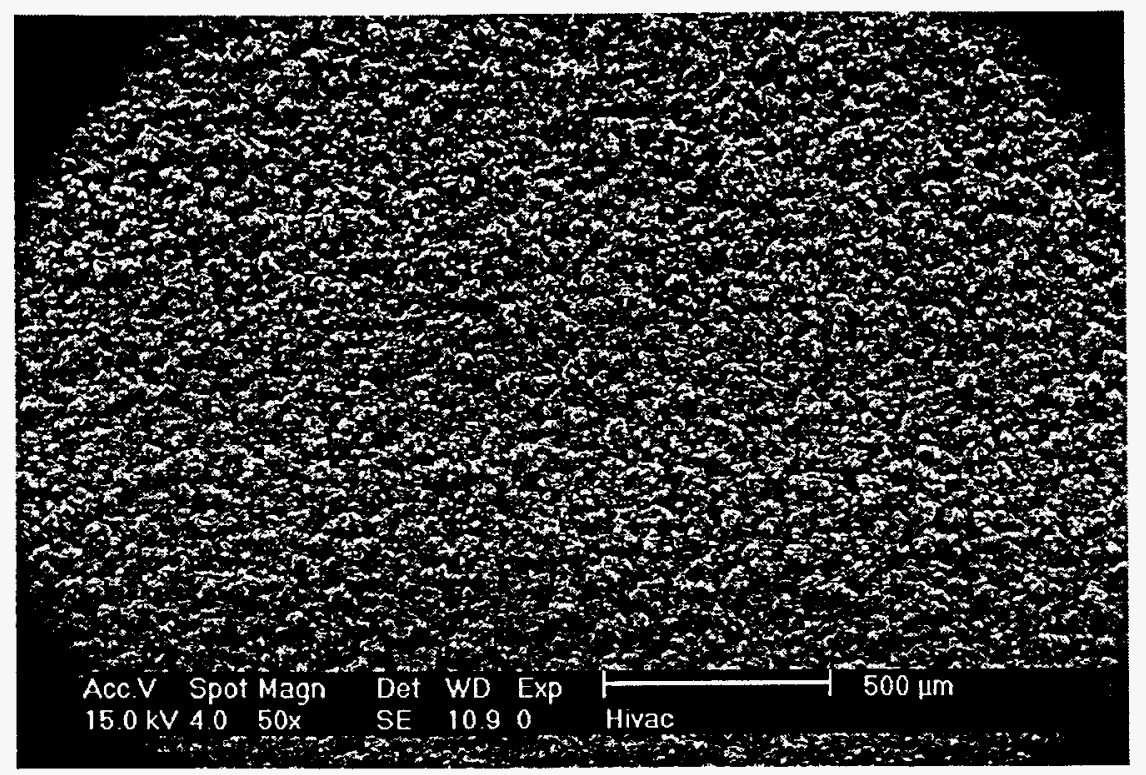

Figure 28(a)

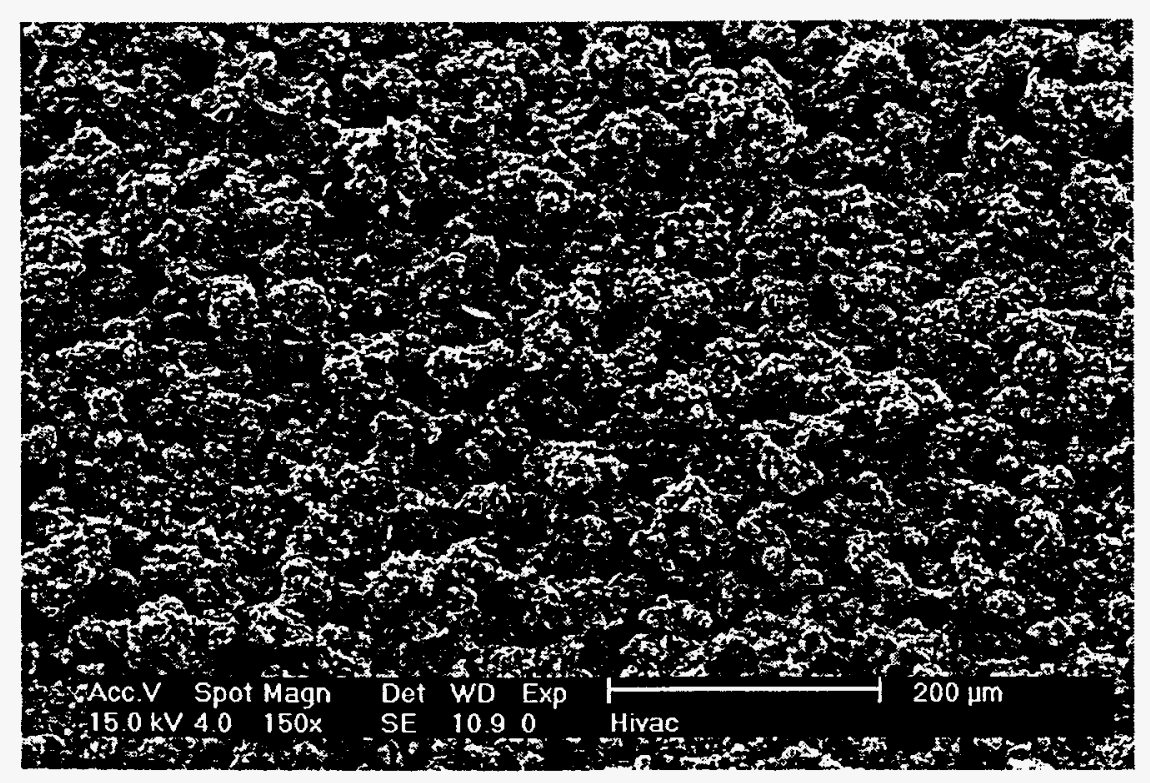

Figure 28(b) 


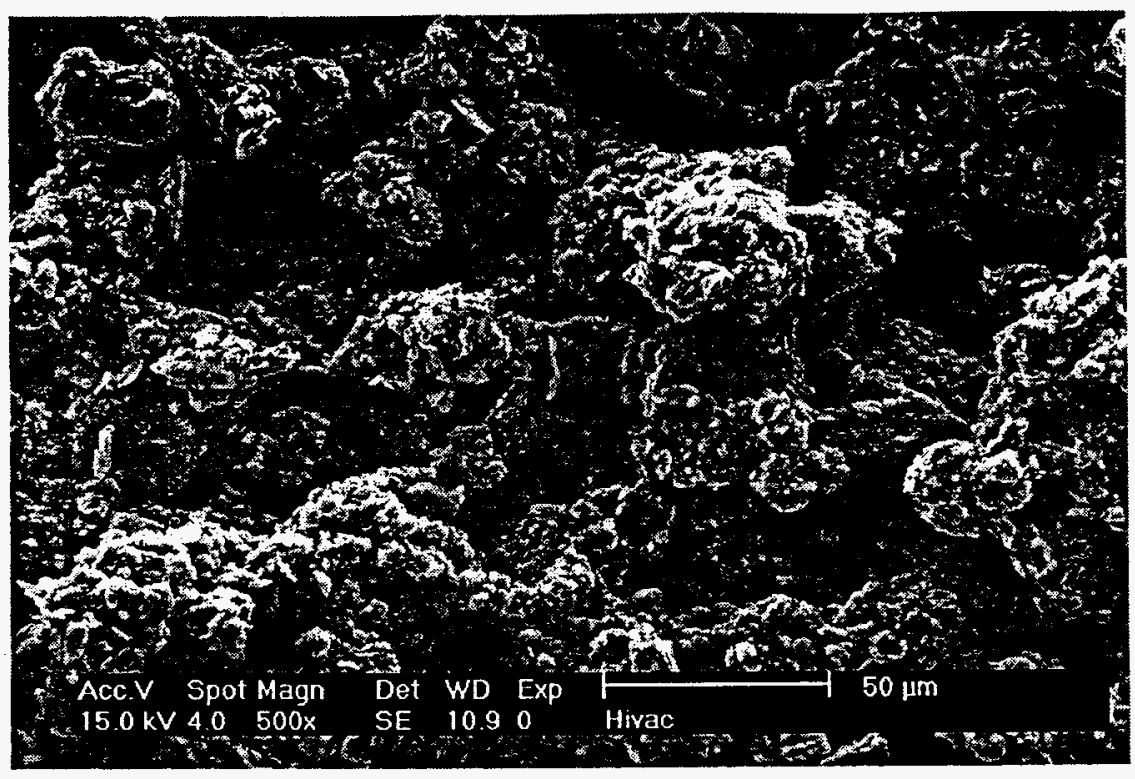

Figure 28(c)

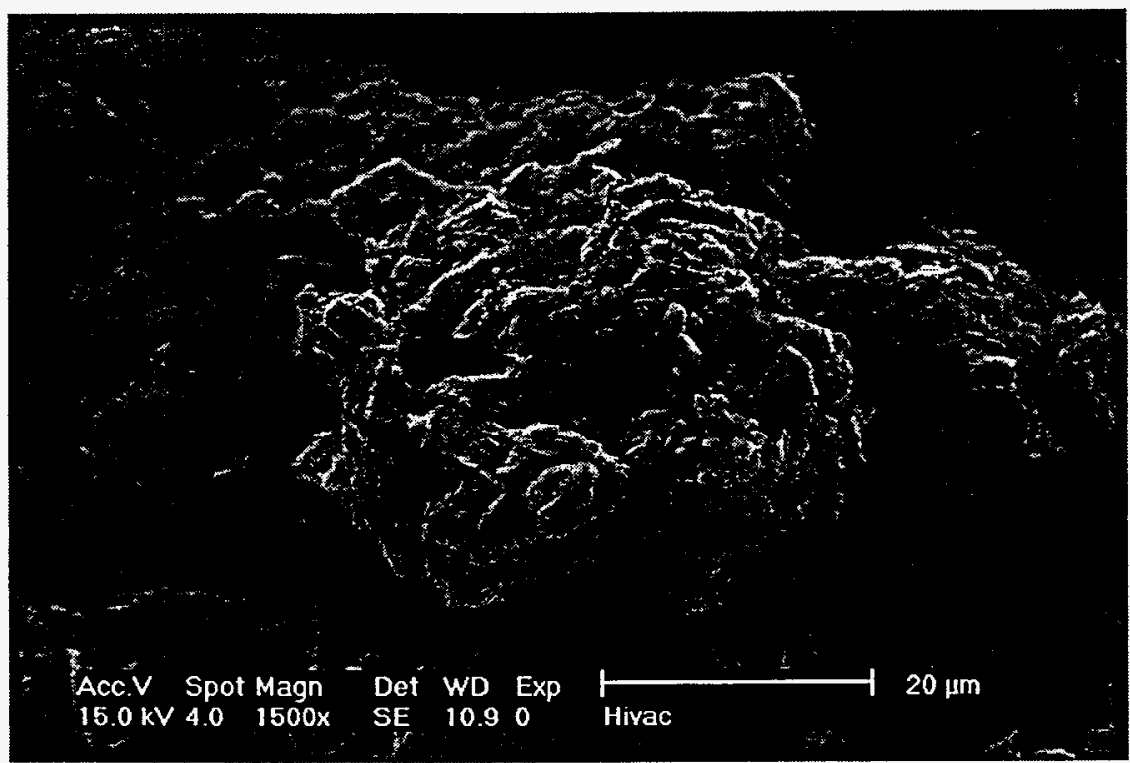

Figure 28(d)

Figure 28 - Morphology of Dycote 11 at Different Magnifications 


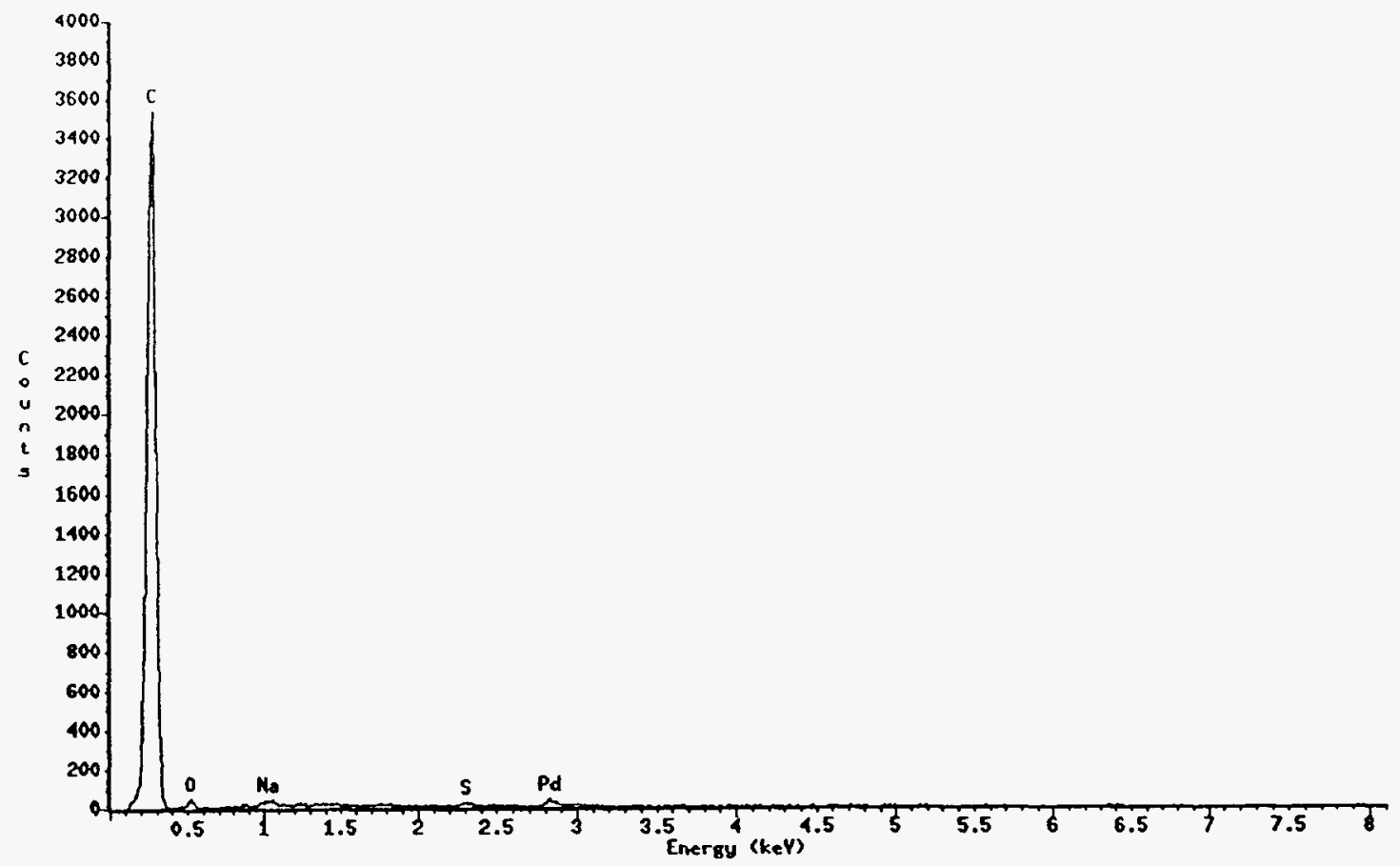

Figure 29 - EDS of Dycote 11 for Area 1 and Area 2 


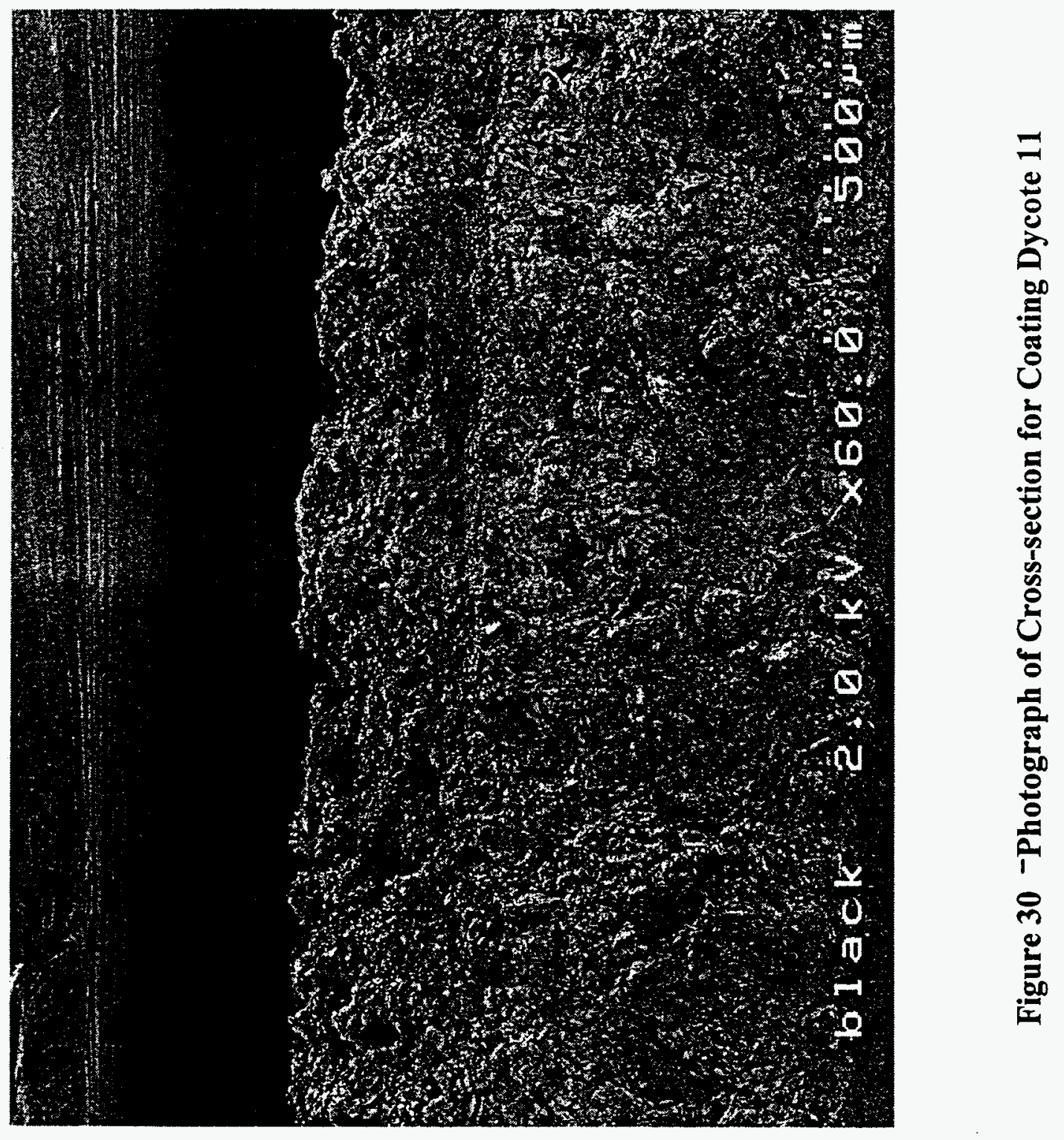




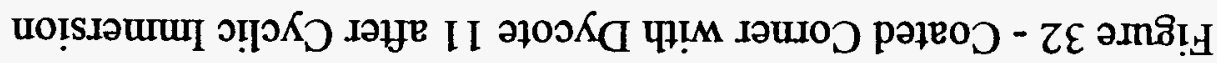

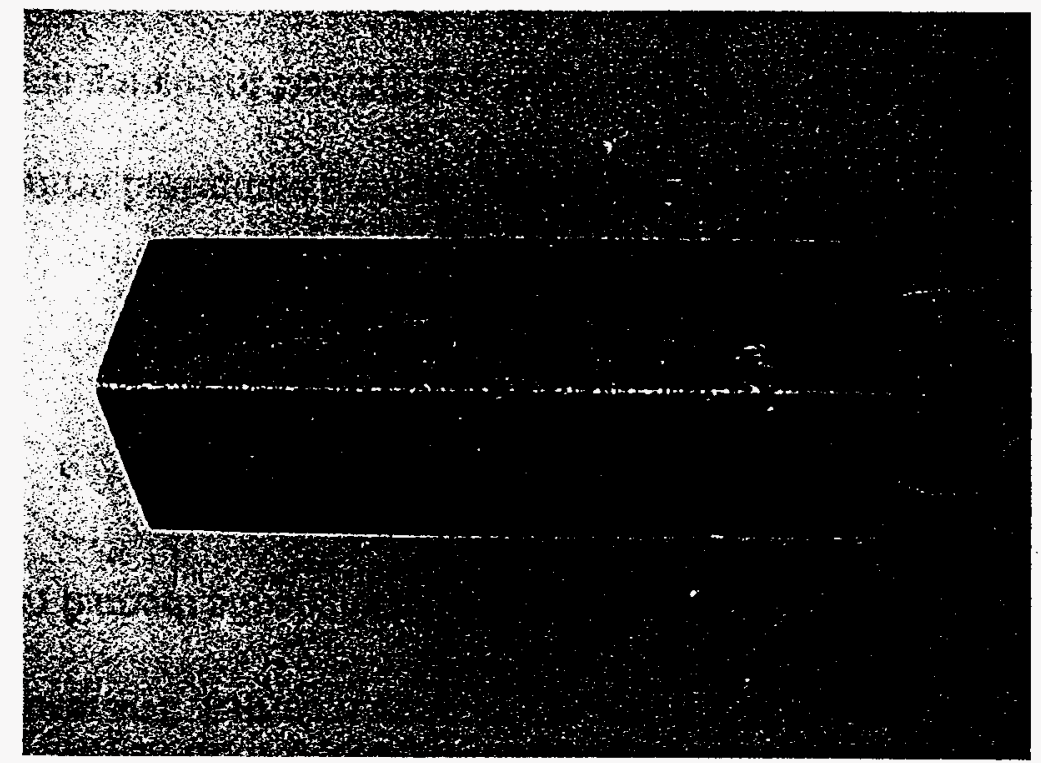

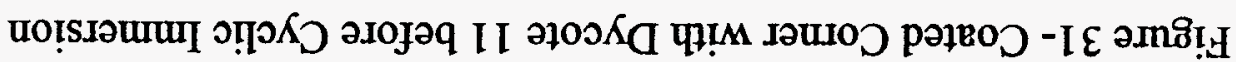

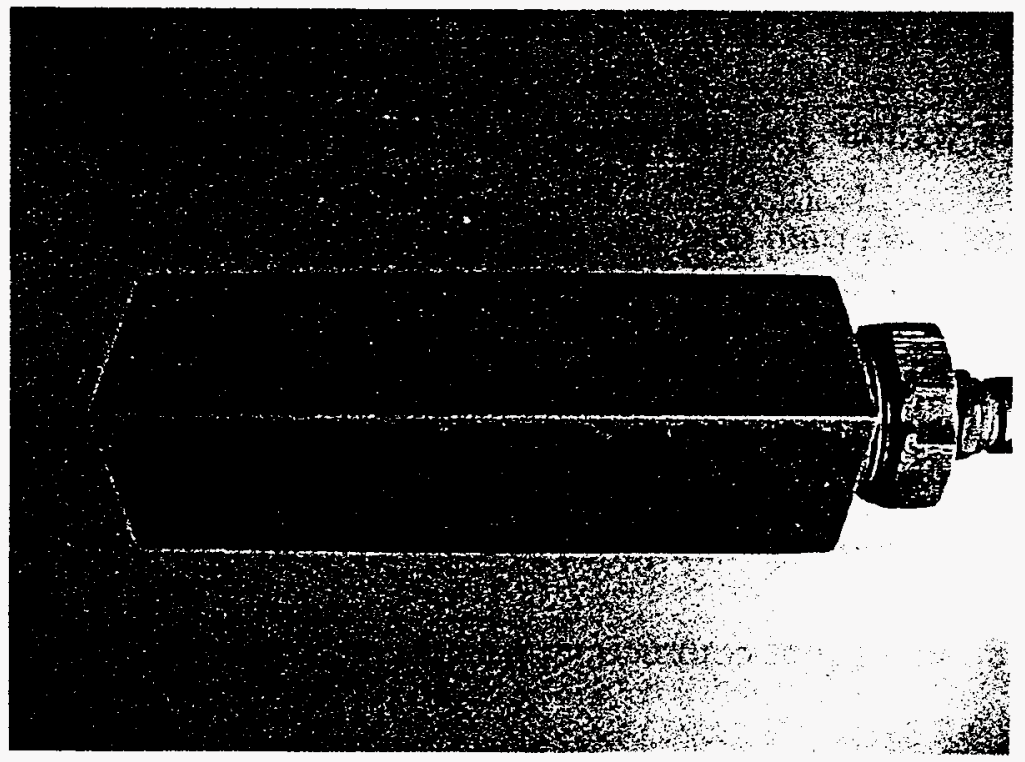


APPENDIX 


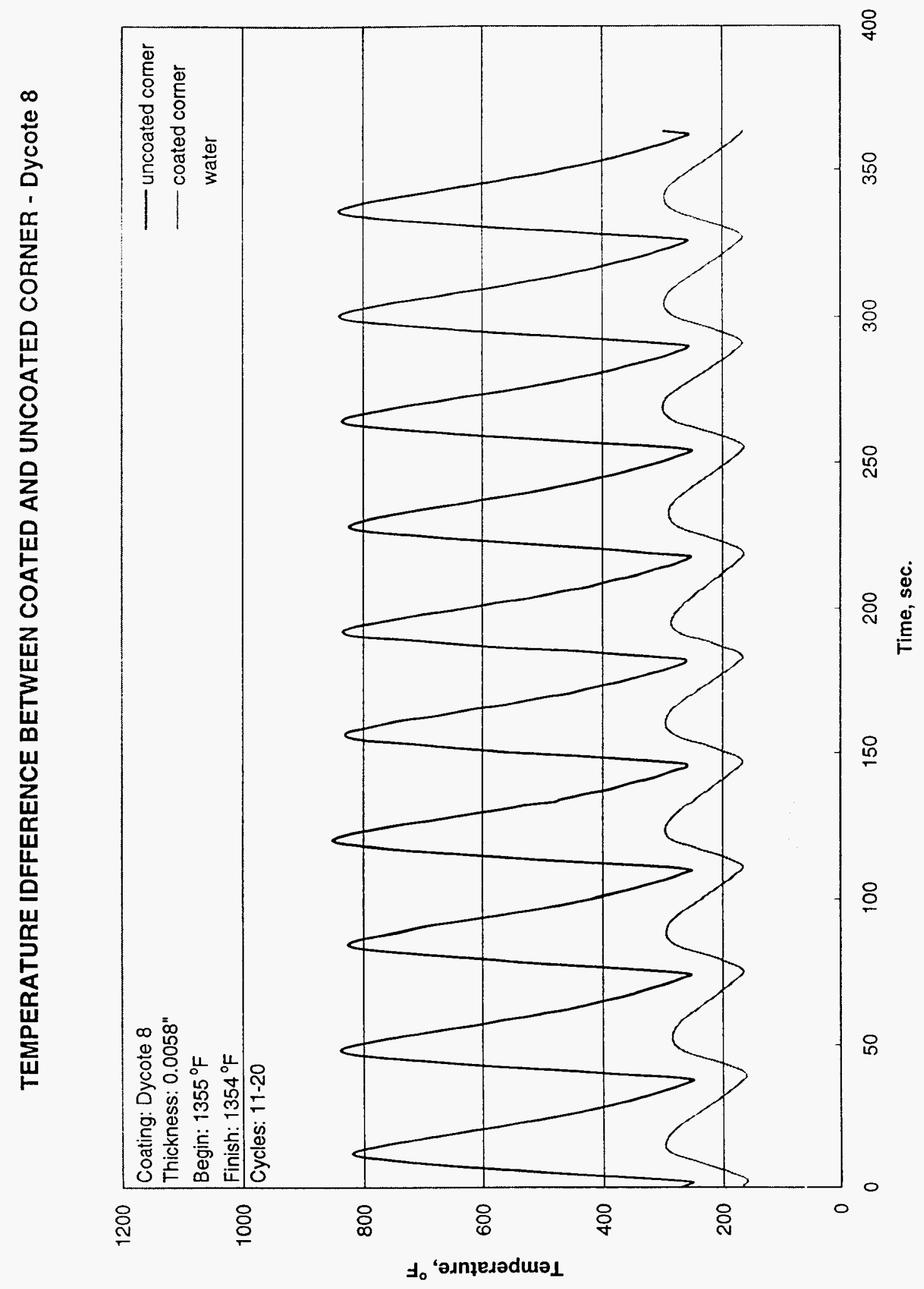




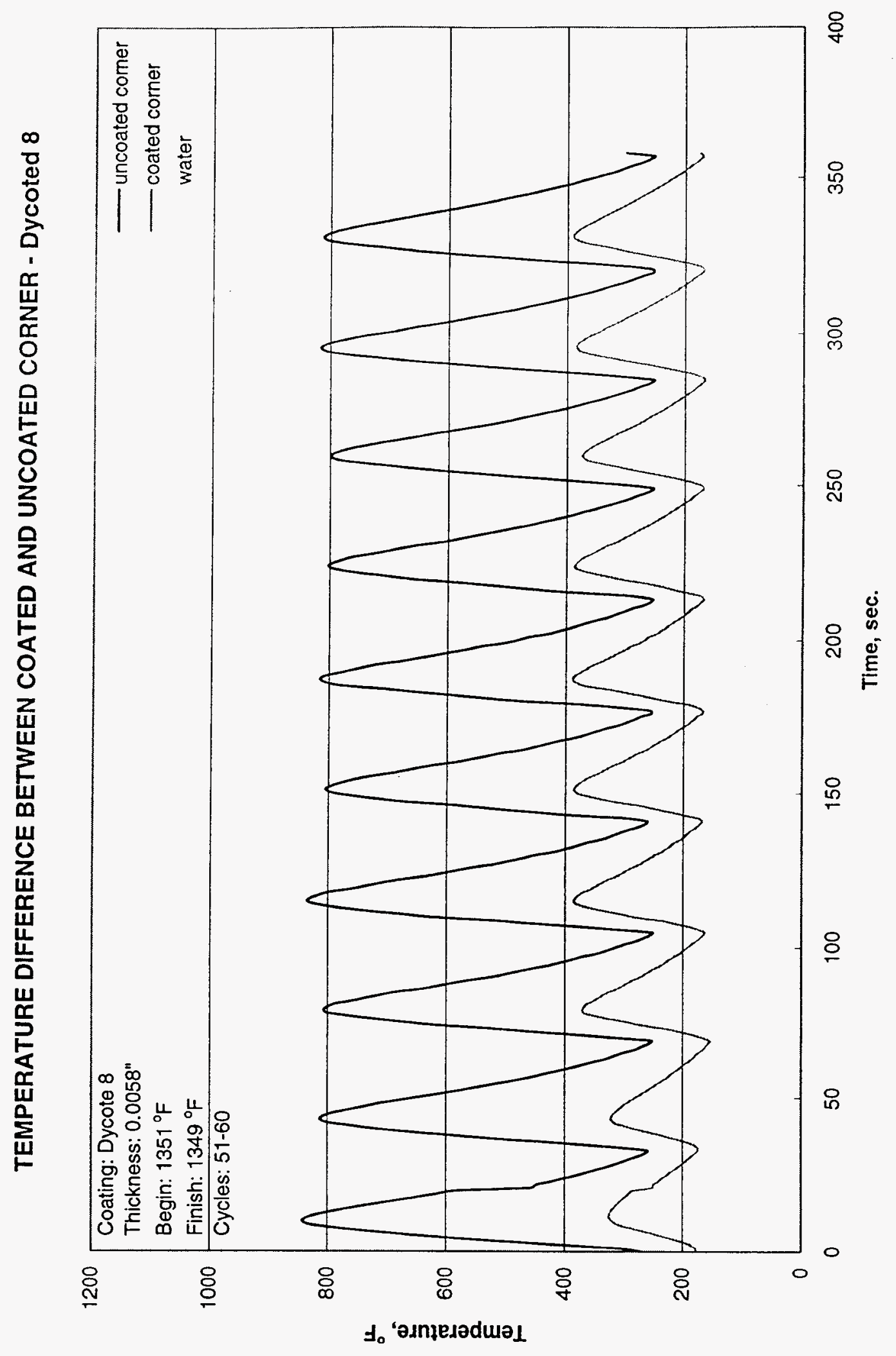




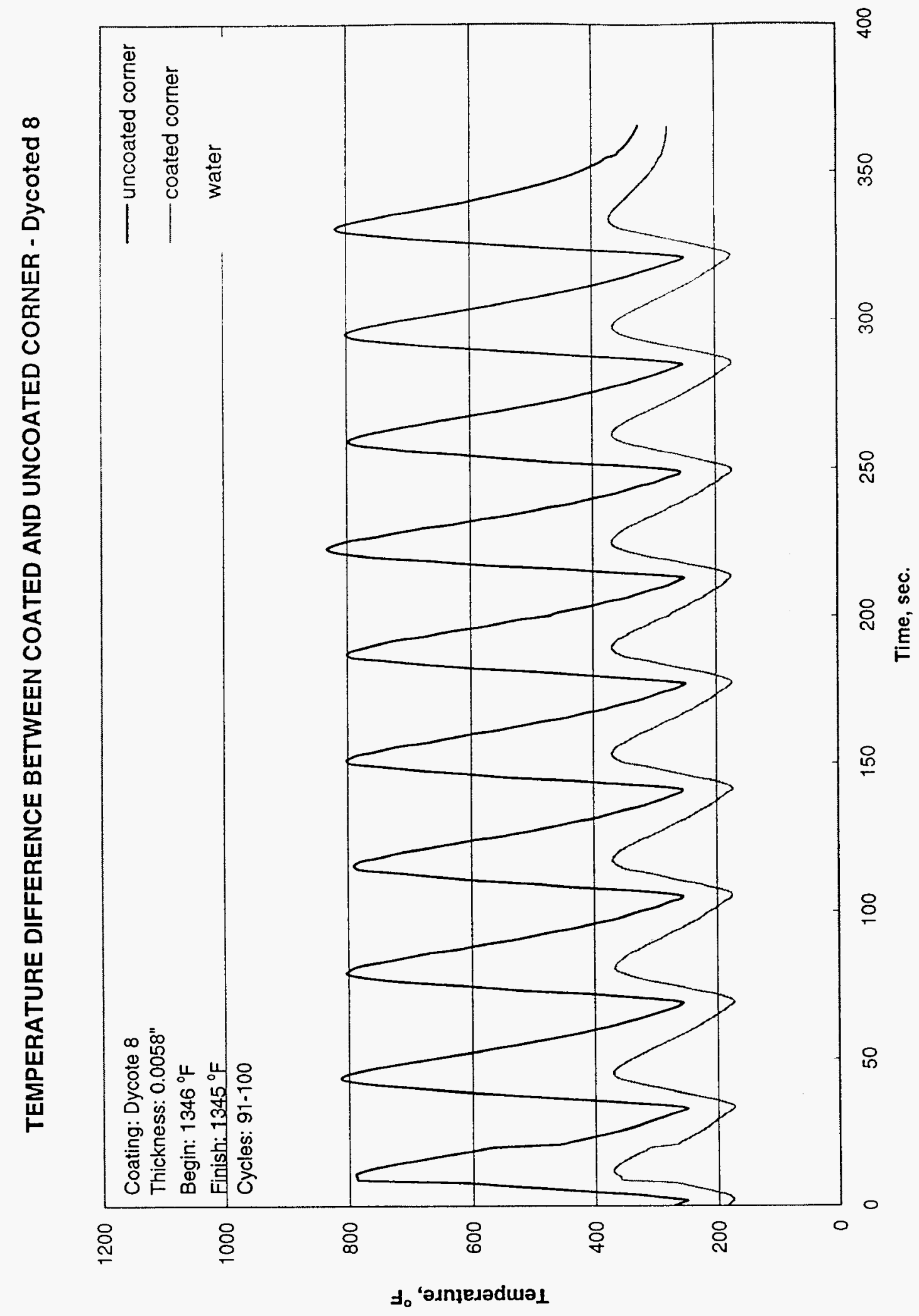




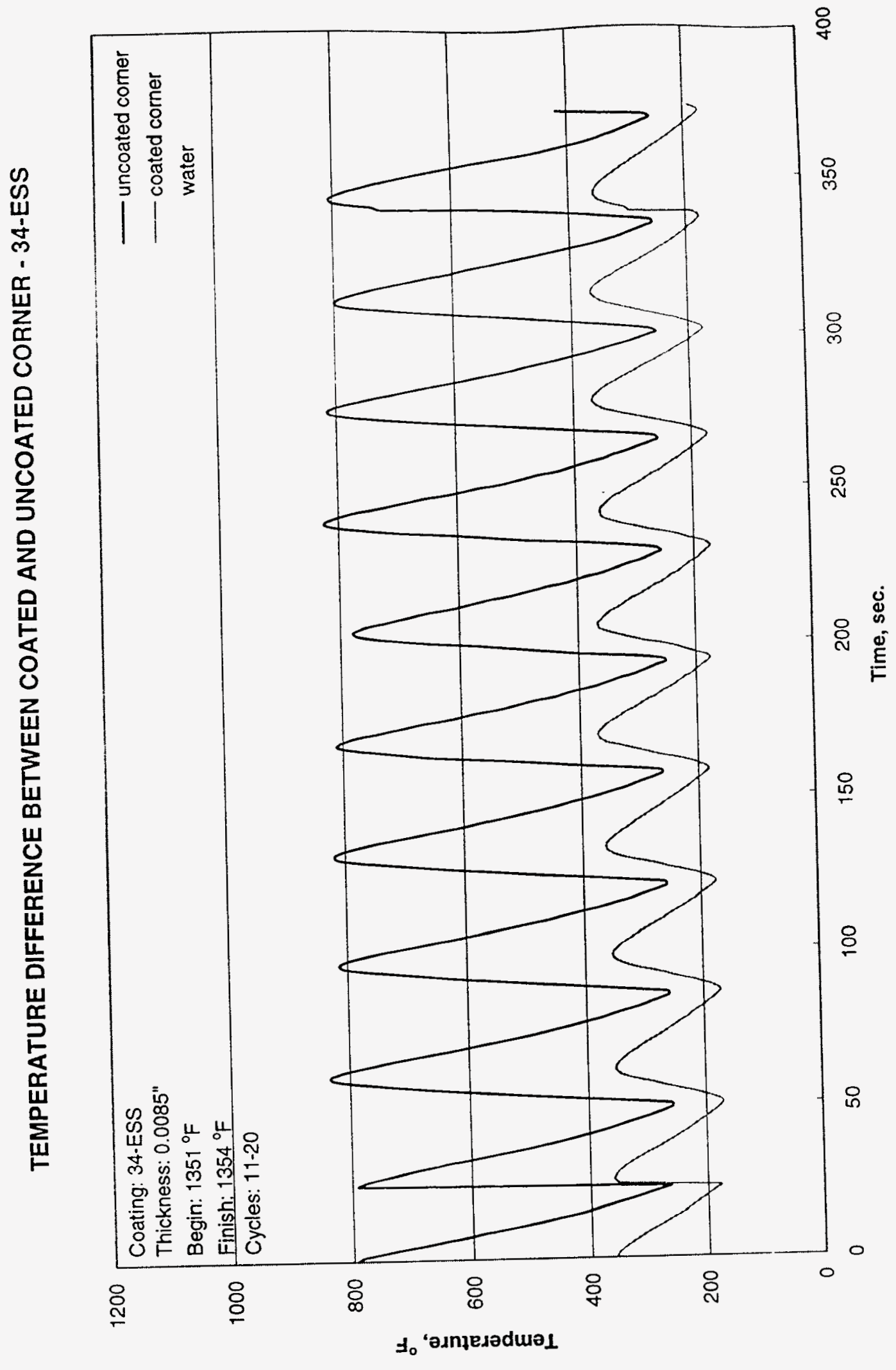




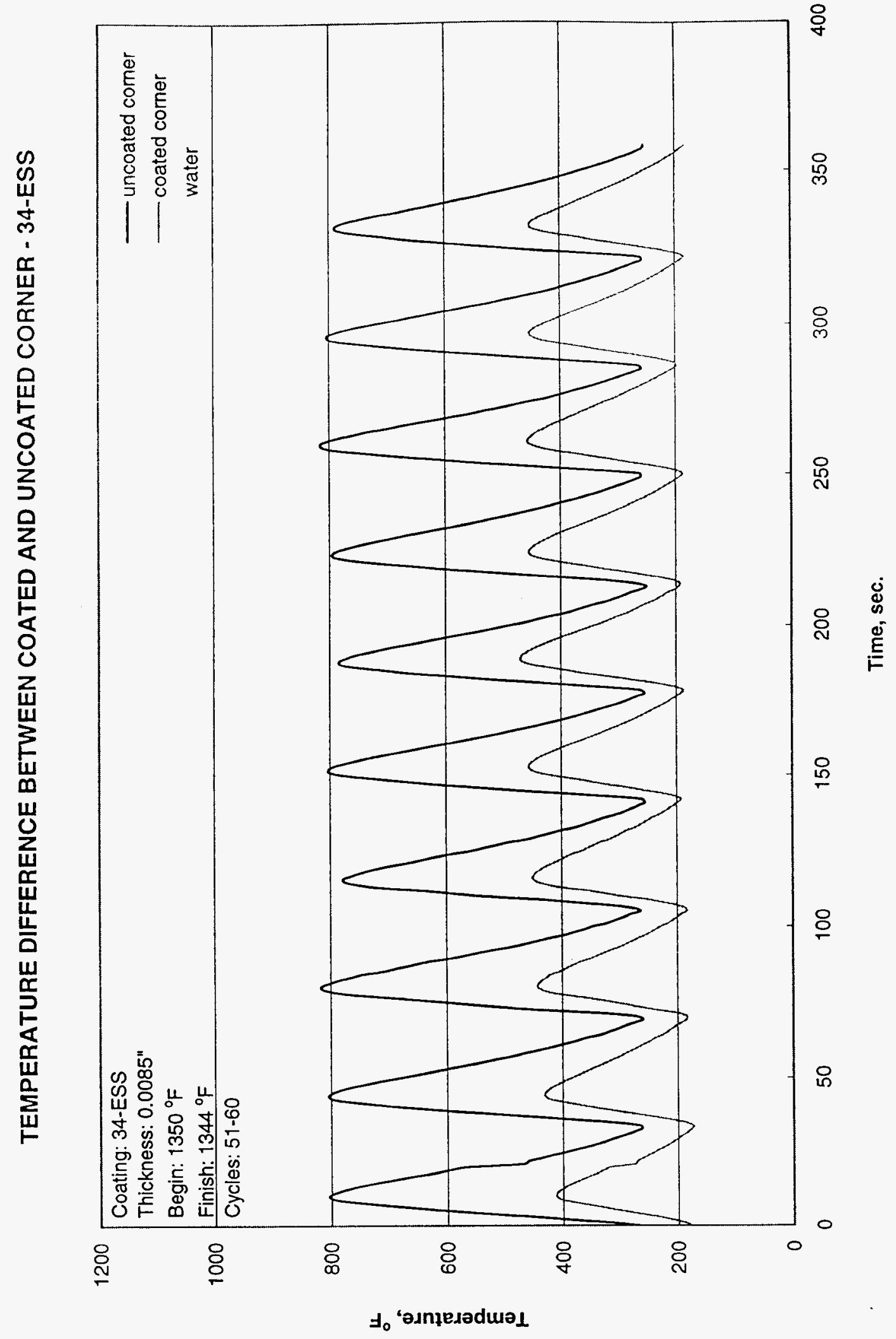




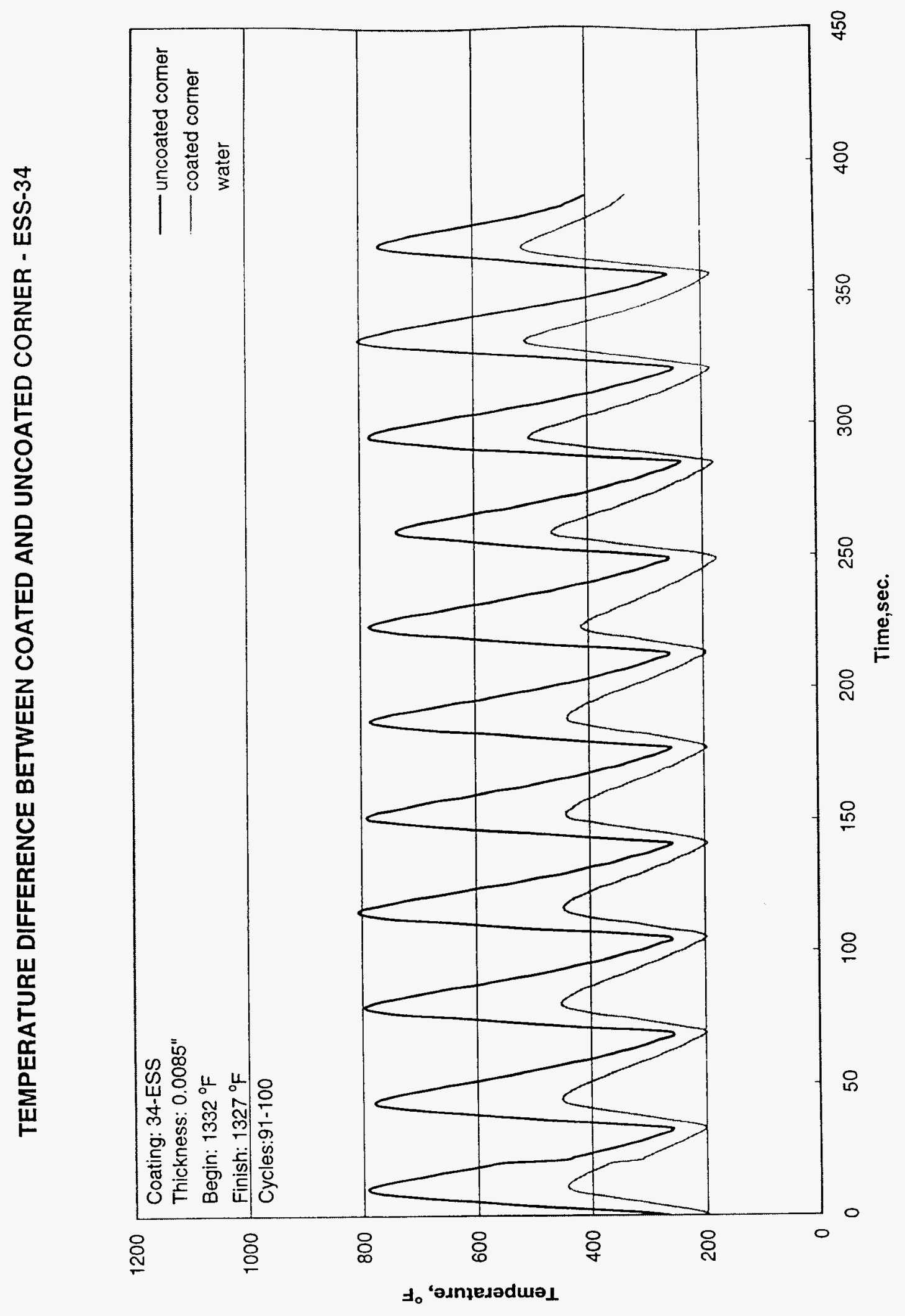




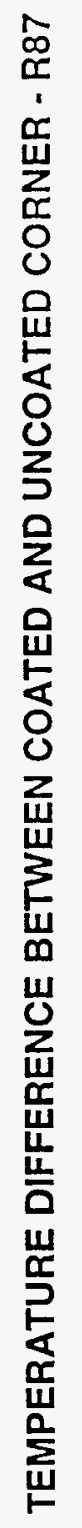

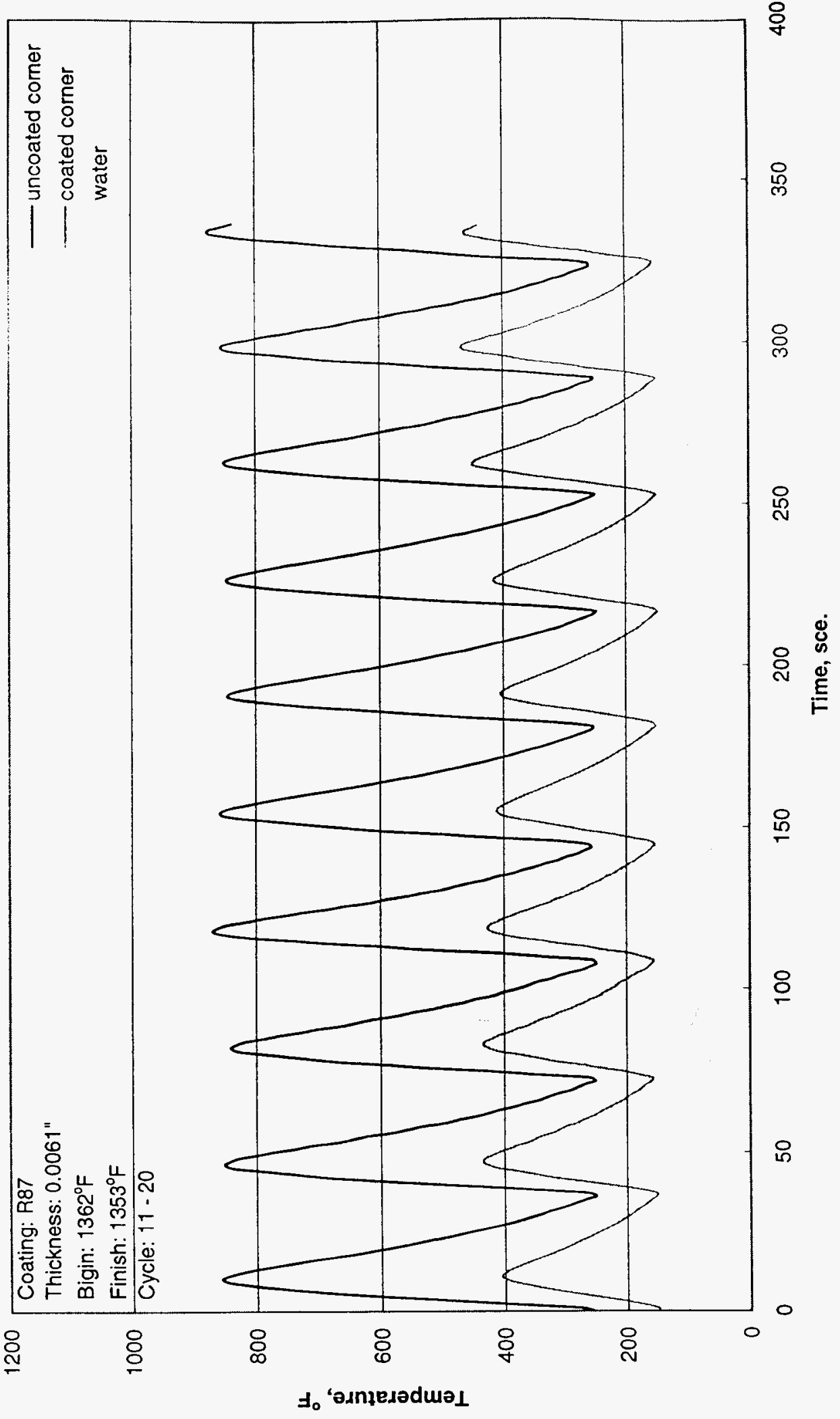




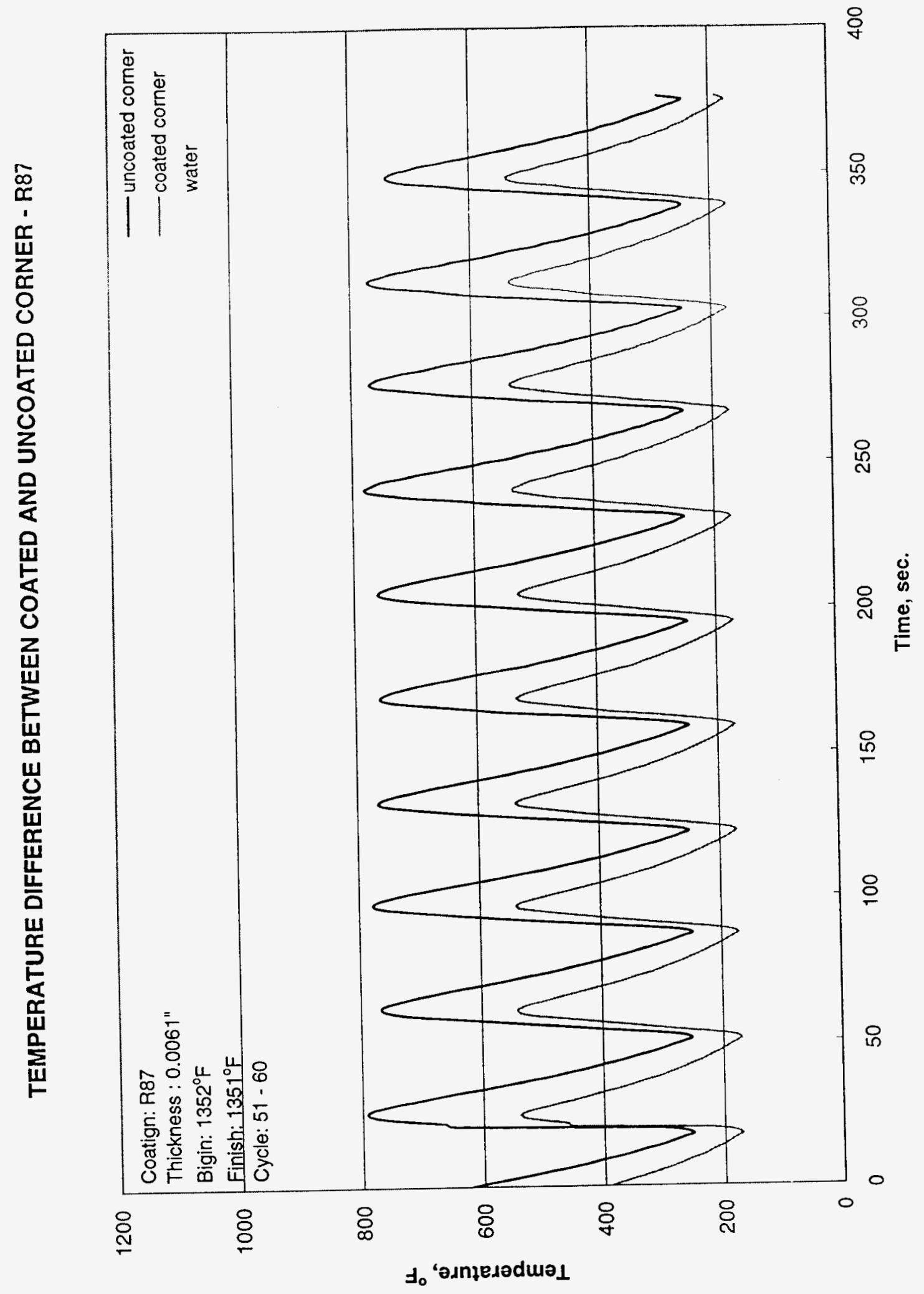




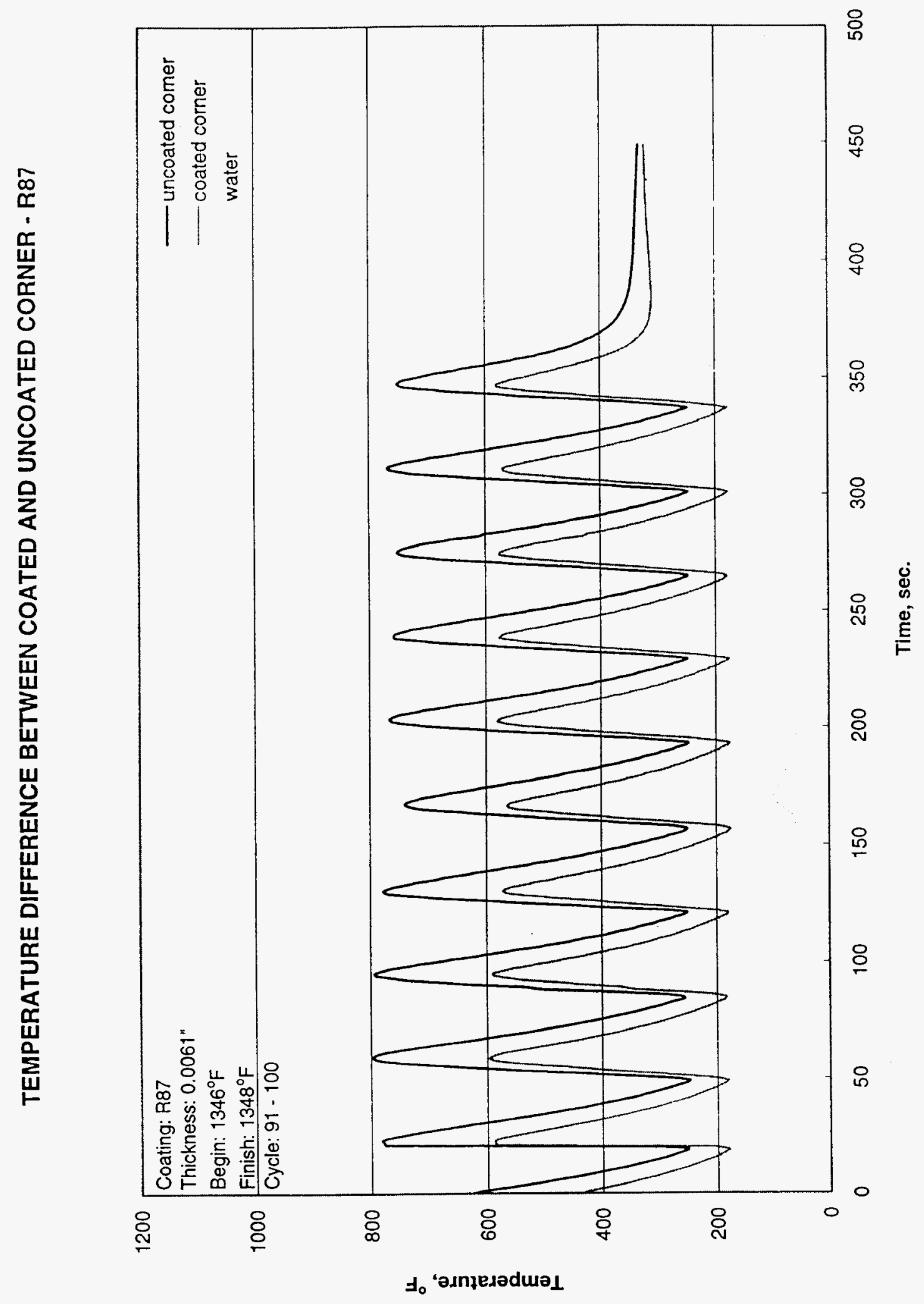


TEMPERATURE DIFFERENCE BTEWEEN COATED AND UNCOATED CORNER - Dycot11

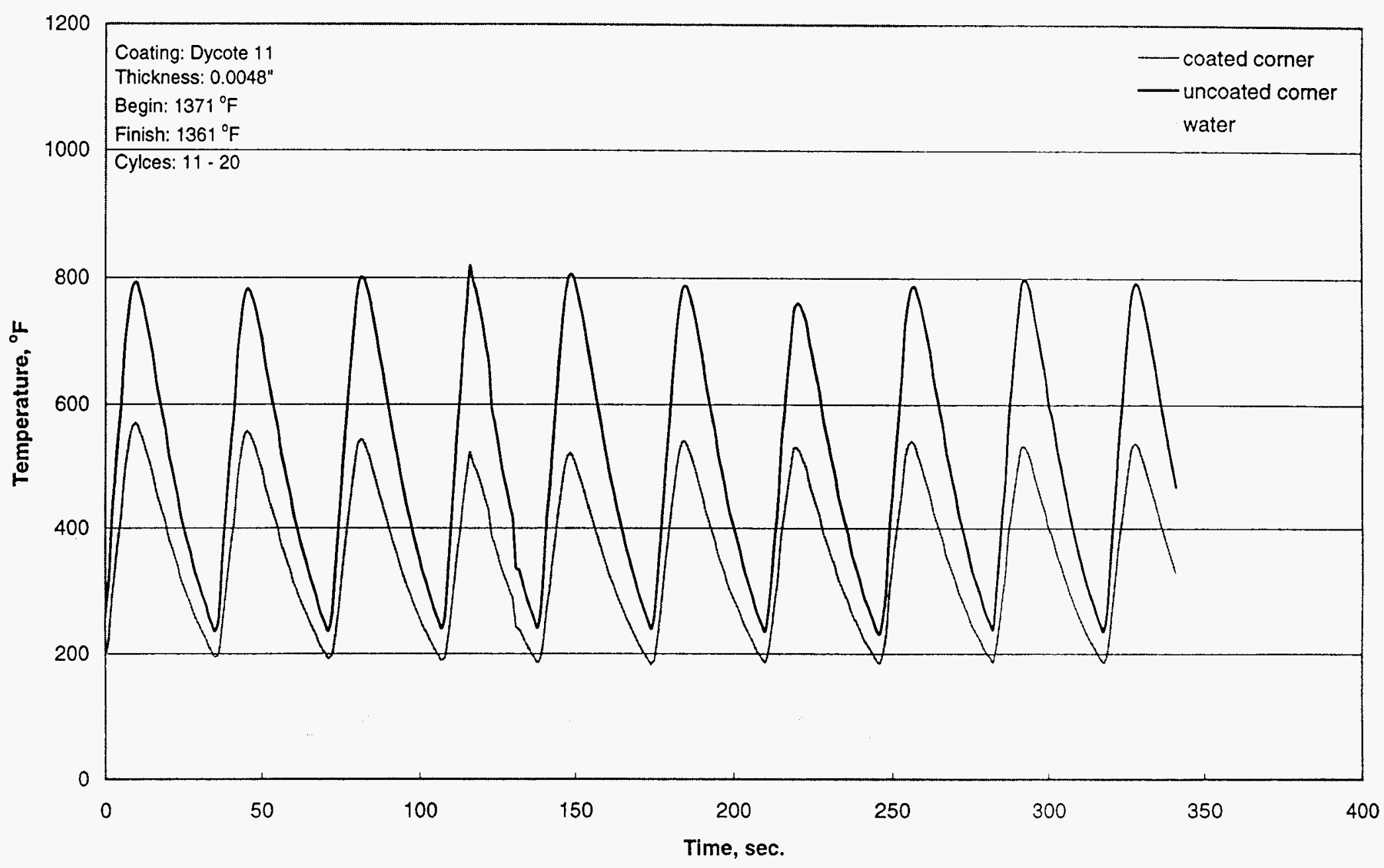




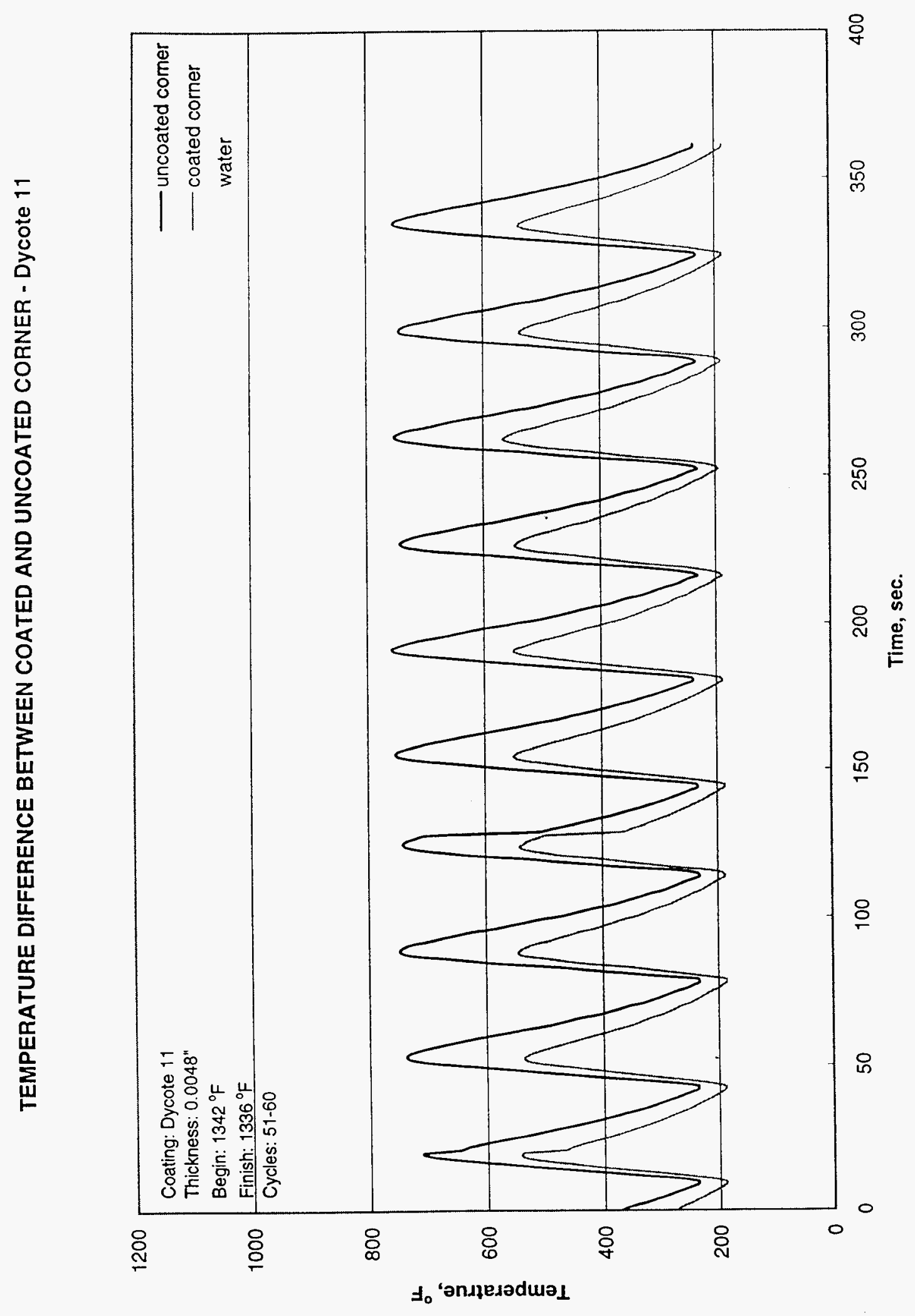


TEMEPRATURE DIFFERENCE BETWEEN COATED AND UNCOATED CORNER - Dycote 11

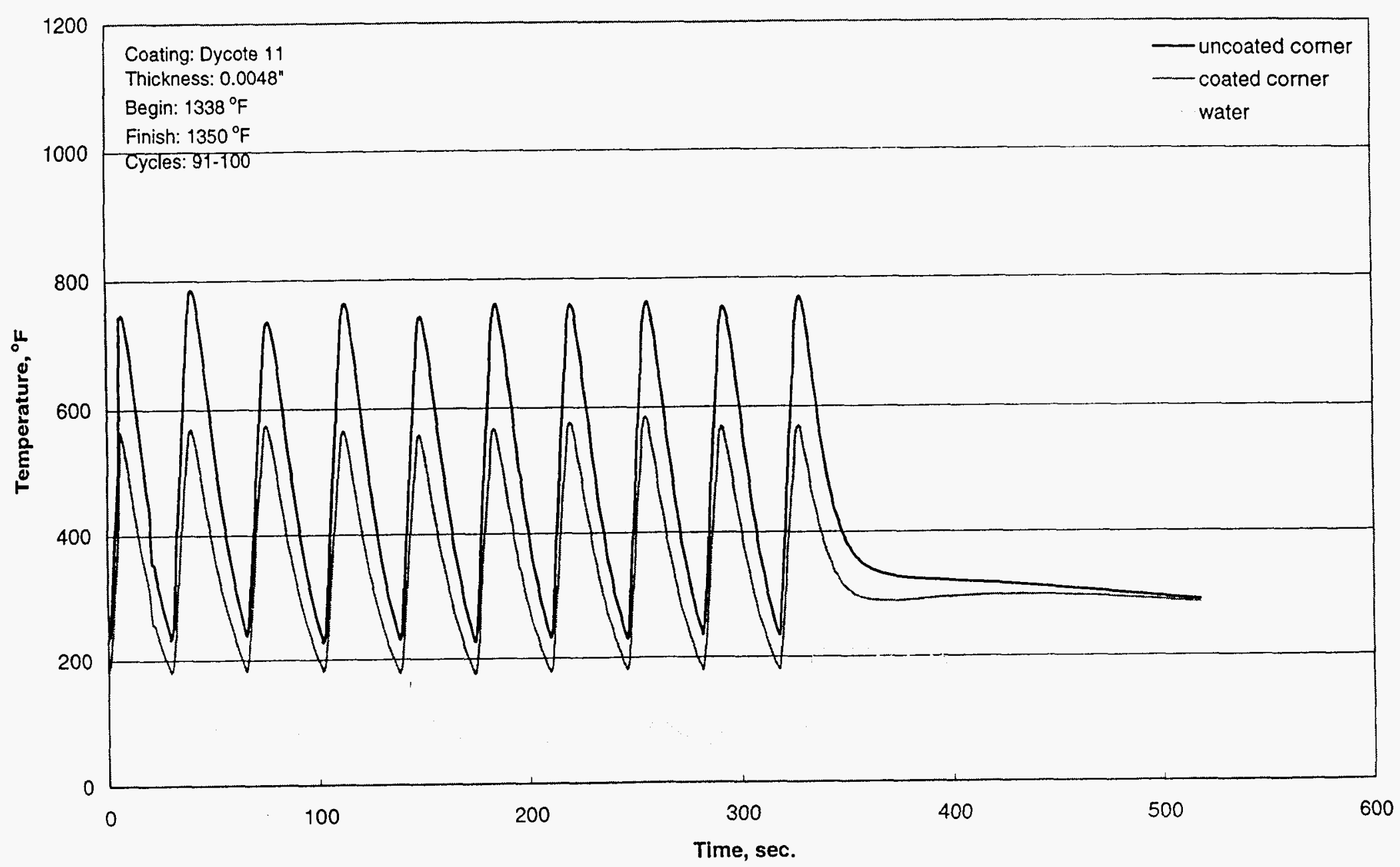

